\title{
METABOLIC MECHANISMS OF VOCAL FATIGUE
}

by

\section{Chayadevie Nanjundeswaran}

BSc Speech and Hearing, All India Institute of Speech and Hearing, India, 2001

MSc Speech and Hearing, All India Institute of Speech and Hearing, India, 2003

Submitted to the Graduate Faculty of

Health and Rehabilitation Sciences in partial fulfillment

of the requirements for the degree of

Doctor of Philosophy in Communication Science and Disorders

University of Pittsburgh

2013 


\section{UNIVERSITY OF PITTSBURGH}

\section{HEALTH AND REHABILITATION SCIENCES}

This dissertation was presented

by

Chayadevie Nanjundeswaran

It was defended on

July 26, 2013

and approved by

Susan Shaiman, PhD, Associate Professor, Communication Science and Disorders

Scott Yaruss, PhD, Associate Professor, Communication Science and Disorders

Jessie VanSwearingen, PhD, Associate Professor, Department of Physical Therapy

Dissertation Advisor: Katherine Verdolini Abbott, PhD, Professor, Communication Science and

Disorders 
Copyright (C) by Chayadevie Nanjundeswaran

2013 


\title{
METABOLIC MECHANISMS OF VOCAL FATIGUE
}

\author{
Chayadevie Nanjundeswaran, PhD, CCC-SLP
}

University of Pittsburgh, 2013

Vocal fatigue is among the most debilitating conditions affecting individuals with voice disorders. Impressions about mechanisms potentially underlying vocal fatigue have varied depending on how fatigue is defined, participants studied, and measures made, thereby impacting the selection of treatment strategies that may alleviate the condition. However, little is currently known about actual metabolic mechanisms of vocal fatigue. The current study aimed to address this issue by investigating the hypothesis that neuromuscular inefficiency, cardiovascular recovery deficits, or both, may play a role in fatigue. The approach replicated well-vetted approaches in exercise physiology.

Metabolic profiles of subjects with vocal fatigue were assessed using gas exchange measures in comparison to two non-fatigue groups: vocally healthy and cardiovascular trained individuals, recruited based on results from a newly vetted questionnaire, the Vocal Fatigue Index (VFI) and laryngeal examination. Participants read out loud at two different loudness levels for a duration of 5 minutes for each task with periods of rest between tasks. Metabolic cost for and recovery time from reading were same across all groups. Oxygen uptake and recovery kinetics (EPOC), ratings of perceived exertion revealed interesting patterns in individuals with vocal fatigue compared to cardiovascular trained individuals in particular. Specifically, slow oxygen uptake kinetics in the vocal fatigue compared to the cardiovascular 
trained group pointed to utilization of anaerobic energy source to meet the demands of the reading task in the vocal fatigue group, suggesting neuromuscular inefficiency. In contrast, rapid oxygen uptake kinetics in the cardiovascular trained group pointed to utilization of aerobic energy sources and greater neuromuscular efficiency. Similarly, a greater number of individuals in vocal fatigue and vocally healthy groups showed an increase in oxygen consumption post reading (EPOC) compared to the cardiovascular trained group, indicating possible cardiovascular recovery deficits in the former groups.

In addition to uncovering potential mechanisms underlying vocal fatigue, including neuromuscular inefficiency and cardiovascular recovery deficits, results from the present study highlight the potential importance of aerobic training to generate aerobic energy required for vocal task demands for both ease of task performance and recovery from it. 


\section{TABLE OF CONTENTS}



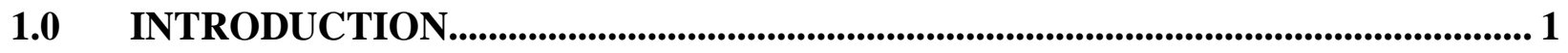

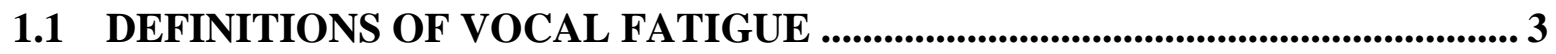

1.2 EXISTING LITERATURE ON VOCAL FATIGUE ......................................... 5

1.2.1 Symptoms and signs of vocal fatigue as a function of subject type ............ 6

1.2.2 Experimentally induced fatigue ....................................................................... 11

1.2.3 Treatment of vocal fatigue..................................................................... 17

1.2.4 Recovery from vocal loading......................................................... 22

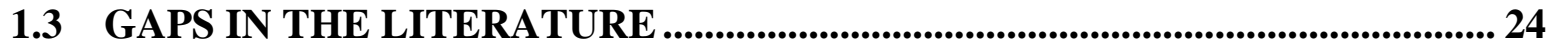

2.0 FATIGUE IN THE EXERCISE PHYSIOLOGY DOMAIN ................................... 26

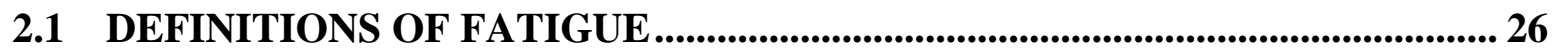



2.3 OXYGEN KINETICS .................................................................................. 29

2.3.1 Central circulation ......................................................................... 30

2.3.2 Peripheral extraction of oxygen (Use) ...................................................... 31

2.3.2.1 Regional oxygen delivery ...................................................................... 32

2.3.2.2 Muscle oxygen extraction........................................................... 33

2.3.3 Take-home point regarding the oxygen kinetic chain ................................ 34

2.3.4 Oxygen Deficit ............................................................................................. 35 


\subsubsection{Excess post exercise oxygen consumption (EPOC) and relation to}

fatigue.

2.4 MEASUREMENT OF MUSCLE FATIGUE ...................................................... 39

2.4.1 Gas exchange analysis....................................................................... 39



2.5.1 Efficiency training.............................................................................................. 42



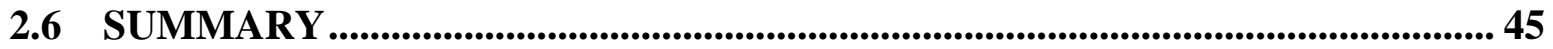

3.0 INTERGRATING EXERCISE PHYSIOLOGY PRINCIPLES WITH THE VOICE

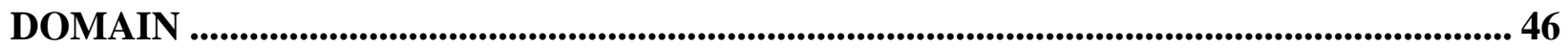

3.1 STATEMENT OF PURPOSE, SPECIFIC AIMS, EXPERIMENTAL QUESTIONS, AND HYPOTHESES ................................................................................... 51

4.0 RESEARCH DESIGN AND METHODOLOGY ..................................................... 56

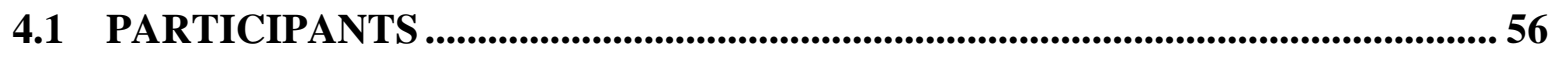

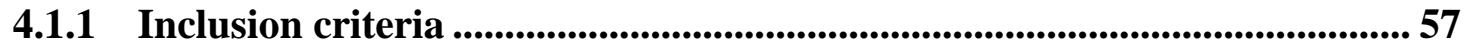

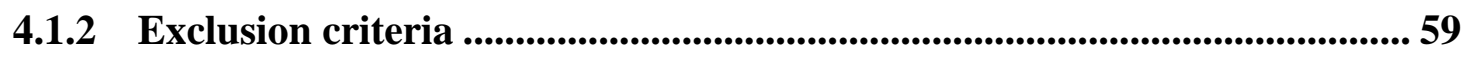

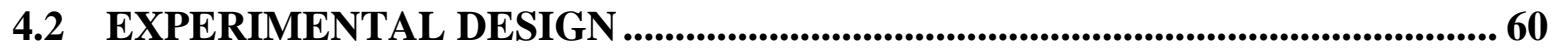

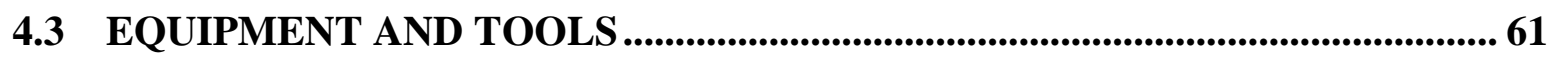

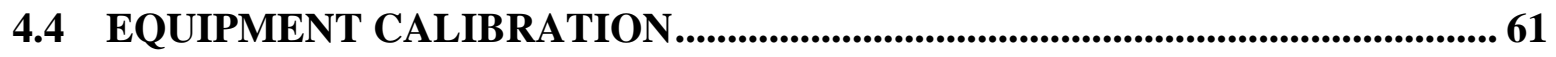

4.5 EXPERIMENTAL PROCEDURES .........................................................6. 62

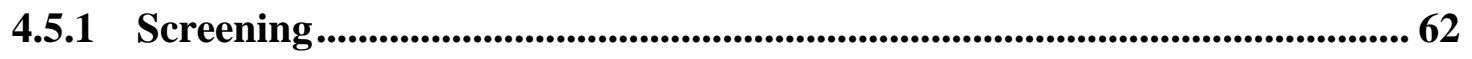

4.5.2 Experimental set-up and training................................................................ 65 
4.5.3 Experimental Procedures ................................................................................. 66

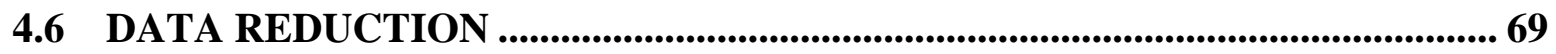

4.6.1 Gross metabolic cost for reading ........................................................................ 69

4.6.2 Extraction of reading metabolic recovery data............................................... 70

4.6.3 Descriptive exploration of raw data (Supplement to S.A. 1)....................... 71

4.6.4 Secondary factors .............................................................................................. 72

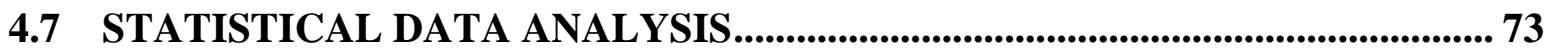

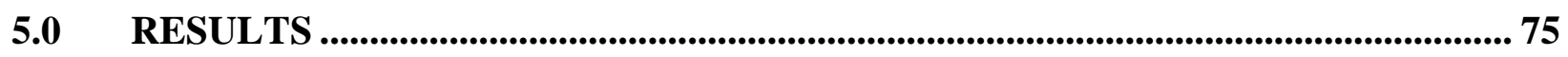

5.1 GROSS METABOLIC COST FOR READING ......................................................... 75

5.2 RECOVERY FROM READING ..................................................................... 79

5.2.1 Recovery time ....................................................................................................... 80

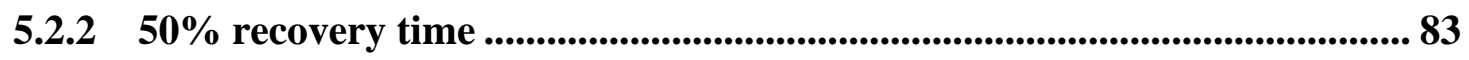

5.3 DESCRIPTIVE EXPLORATIONS..................................................................... 84

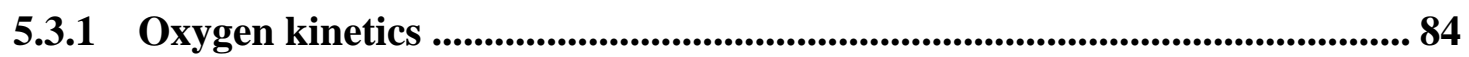

5.3.2 Excess post-exercise oxygen consumption (EPOC).......................................... 87

5.4 SECONDARY AIMS .................................................................................................... 87

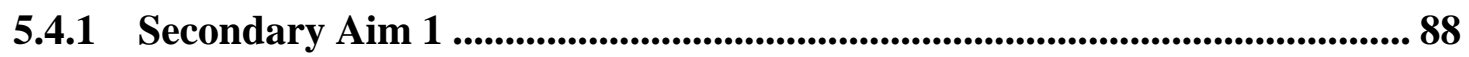

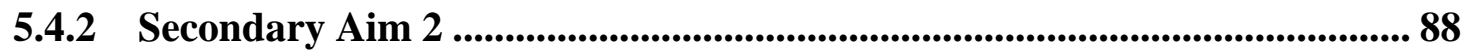

5.4.3 Secondary Aim 3 ................................................................................................ 89

5.4.4 Secondary Aim 4 .............................................................................................. 90

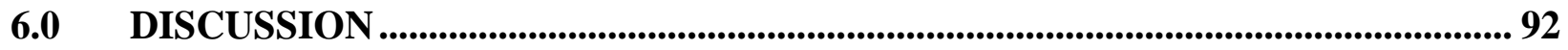

6.1 SUMMARY AND CONCLUSIONS........................................................................... 99

6.2 LIMITATIONS AND FUTURE DIRECTIONS ................................................... 101 
APPENDIX A. RECRUITMENT FLYER - HEALTHY FEMALES

APPENDIX B. RECRUITMENT FLYER - VOICE PROBLEMS 105

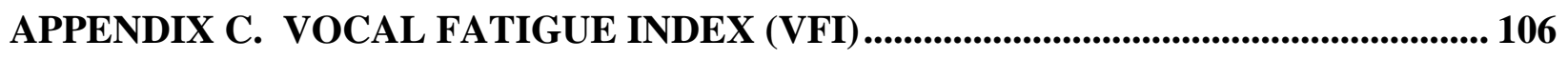

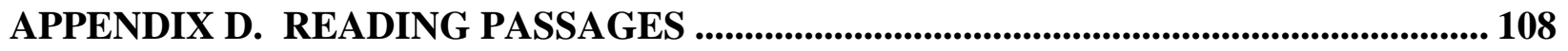

APPENDIX E. BORG RATINGS OF PERCEIVED EXERTION ........................................121

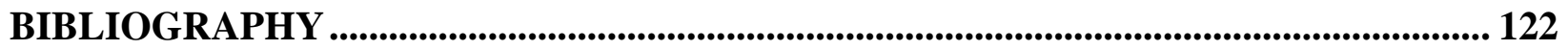




\section{LIST OF TABLES}

Table 1-1 Summary of studies on symptoms and signs of vocal fatigue as a function of subject

Type

Table 1-2 Summary of studies on experimentally induced fatigue ..................................... 12

Table 1-3 Summary of treatment studies in vocal fatigue...................................................20

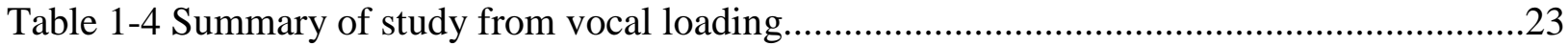

Table 4-1 Task order of the experimental protocol for the day ............................................66

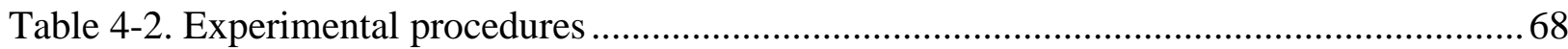

Table 4-3 Dependent and independent variables for specific aim 1 ......................................73

Table 5-1. Mean (SD) values of gross metabolic cost for two different loudness levels with their corresponding mean (SD) dB sound pressure level (SPL) across the three groups of participants (vocal fatigue, vocally healthy and cardiovascular trained)..............................................76

Table 5-2. Descriptive data (mean, SD) for conversational and loud Reading Recovery Time in Seconds for the Three Groups (Vocal Fatigue, Vocally Healthy and Cardiovascular Trained

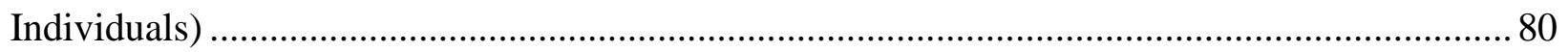

Table 5-3. Distribution of 50\% Recovery at One and Two Minutes Post Energy Expenditure for



Table 5-4. Number (Percent) of Individuals with a Rise in Oxygen Consumption (ml/kg/min)

Post Reading (EPOC) across the Three groups ............................................................ 87 
Table 5-5. Descriptive Data for Perceived Effort (RPE) for both Conversational and Loud Reading across the Three Groups (Vocal Fatigue, Vocally Healthy, and Cardiovascular

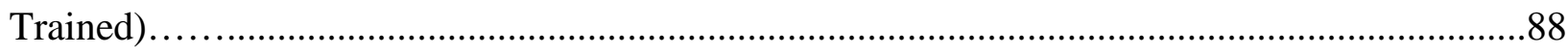

Table 5-6 Descriptive Data (Mean, SD) for Heart Rate across the Three Groups (Vocal Fatigue,

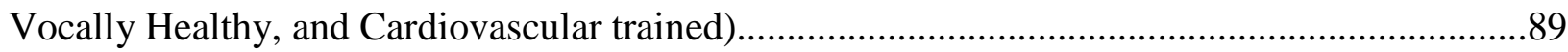

Table 5-7. Descriptive Data (Mean and SD) for Vocal Efficiency (dB/cmH20 x l/s) across Three Groups (Vocal Fatigue, Vocally Healthy, and Cardiovascular trained)........................................90

Table 5-8. Descriptive Data (Mean and SD) for Vocal Efficiency (dB/cmH20 x 1/s) across Three Groups removing Outlier in the Vocal Fatigue Group (Vocally Healthy, Vocal Fatigue, and Cardiovascular trained) 90 


\section{LIST OF FIGURES}

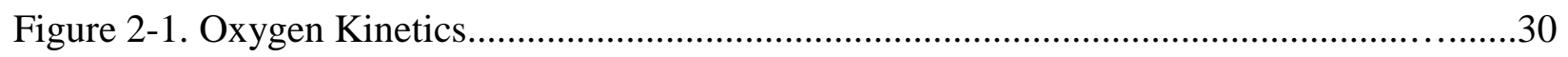

Figure 2-2. Diffusion of oxygen from capillary to muscle fiber............................................33

Figure 2-3. Oxygen uptake response at the onset of exercise...............................................36

Figure 3-1. A conceptual Framework of Vocal Fatigue......................................................49

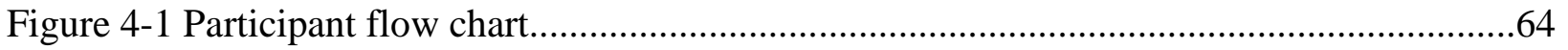

Figure 5-1 Metabolic cost (ml/kg-w) for conversational and loud reading across the three groups; vocal fatigue, vocally healthy and cardiovascular trained..................................................77

Figure 5-2. Metabolic cost (ml/kg-w) across the three groups; vocal fatigue, vocally healthy and cardiovascular trained collapsed across loudness conditions.............................................78 Figure 5-3. Average metabolic cost (ml/kg-w) and variability for conversational and loud reading

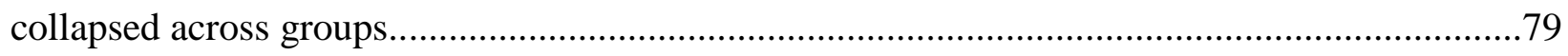
Figure 5-4. Average and variability of recovery time (sec) for conversational and loud reading across the three groups; vocal fatigue, vocally healthy and cardiovascular trained....................81 Figure 5-5. Average variability in recovery time (sec) for the three groups- vocal fatigue, vocally healthy and cardiovascular trained, collapsed across loudness conditions. .82 Figure 5-6. Average and variability of recovery time (sec) for conversational and loud reading, collapsed across groups. 
Figure 5-7. Line graph demonstrating typical pattern of oxygen kinetics in cardiovascular trained individuals - with rise time starting at 10.55 at the onset of reading task and a plateau achieved at

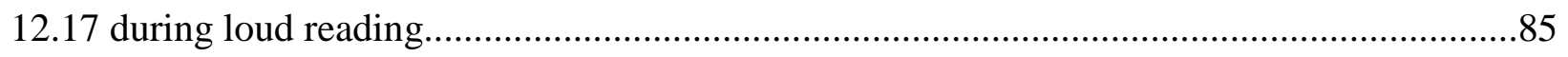

Figure 5-8. Line graph demonstrating atypical oxygen kinetics indicating a double plateau in a

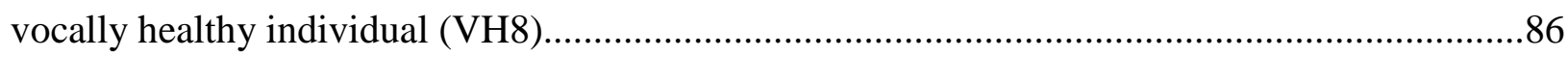

Figure 5-9. Line graph illutrating one pattern of oxygen kinetics observed in six individuals with vocal fatigue (e.g., VF12) showing no change in oxygen consumption from baseline to task

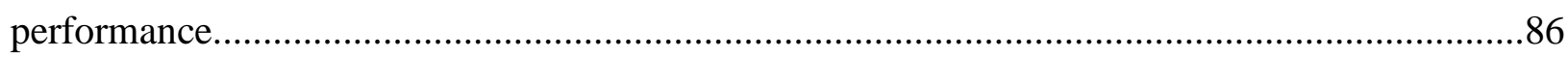
Figure 5-10. Line graph illustrating a second pattern of oxygen kinetics observed in other individuals with vocal fatigue (e.g, VF7) with rise time starting at 12.27 and no plateau achieved

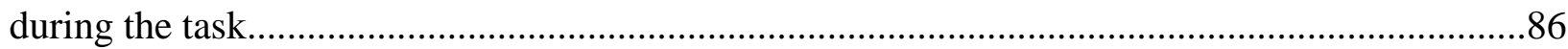




\section{LIST OF EQUATIONS}

Equation 2-1. Fick’s equation summarizing oxygen consumption.......................................30

Equation 2-2. Equation summarizing cardiac output..........................................................31

Equation 2-3. Equation demonstrating law of hemodynamics ..............................................32 


\section{PREFACE}

This $\mathrm{PhD}$ path and my dissertation has been a long journey, but one that is fruitful, productive, and worthwhile. Thank you to all that made this dissertation possible! I would like to thank my committee members for their constant support and guidance throughout this process. Kittie, thank you for providing me with such a great learning environment. I have always looked up to you and admired your dedication. You are an inspiration and I hope to continue in your path.

Jessie, this would not have happened without you. I will always remember our long hours of intellectually stimulating discussions on fatigue. I have learned a lot from you and I hope to be committed to students like you have been to me. Sue, thank you for always helping me think about my priorities when I doubted my abilities. Dr. Yaruss, thank you for your thoughtful suggestions throughout my doctoral program and your commitment. Dr. Brown, Thank You!

Jackie, thank you for your friendship, your insights through this process and always encouraging me. Edie, thank you for your willingness to jump on board and for being a great friend.

My colleagues at ETSU, Marc, Kerry, Pat, Pepper, Teresa, I could not have done this without your constant encouragement and support. Brenda, thank you for always being there for me. Your constant encouragement, support, and thoughtful suggestions have helped me through this process. Jacek, thank you for your expert statistical consultation and readiness to help me 
anytime. Lynn, thank you for being that person to boost my personality and for looking out for me.

April and Kimberly, you have been with me every step of this process. We have spent time together sharing $\mathrm{PhD}$ talks, motherhood talks, and life in general. Thank you for lifting my spirits when I needed it the most. You two are gems in my life.

Sara and Kaveri, thank you for taking care of the boys on my numerous trips to Pittsburgh during this period and easing my mind.

Maria and Nicole, you have been my helping hands and guide through this process. You both kept me on my toes with research and were good listeners anytime I wanted to vent. I cherish our times spent in the lab, our talks, and our trips and visits. Thank for being such wonderful senior mentors. Labmates Doug, Amanda, Aaron, Leah, thank you for your support through my long distance $\mathrm{PhD}$ process and rooting for me.

Vijay, there are no words to describe your support and love through this entire process. I know this was not easy. Thank you for listening, understanding, and putting up with my PhD tantrums!! Congratulations on your second $\mathrm{PhD}$ and now we can have our life back!!! Nishanth, my pappuch, thank you for reminding me that there is more to life. Your smile, your cuddles, and when you say "I love you" remind me how thankful I am to be a mother. I hope all your PhD talk gets you wherever you want and what you want to achieve in life!

Amma and Appa, I am who I am because of you. I have learned from you that hard work, dedication, courage, and belief in self surely pays off. Thank you for letting me make the choices I made and trusting in me. You have been there for me when I needed you in the literal sense and emotionally. I am finally done!!!!!

To all my family members, thank you for rooting for me to get through my PhD. 


\subsection{INTRODUCTION}

“I don’t feel like talking any further. I feel like I have to stop talking right now. My throat aches. My voice feels tired. I feel like I have to strain to produce my voice”.

These are common statements one can hear from individuals with voice problems. Voice disorders are the most common communication disorder across the life span. Most reports indicate voice problems occur in approximately 3-9\% of the general population at any given point in time (Verdolini \& Ramig, 2001). These types of statements are typically made by people who experience what clinicians characterize as “vocal fatigue.” So-called vocal fatigue is among the most frequent and debilitating symptoms affecting individuals with voice problems, and can affect social and occupational functioning, sometimes profoundly (Koufman \& Blalock, 1988; McCabe \& Titze, 2002; Smith, Gray, Dove, Kirchner, \& Heras, 1997; Welham \& Maclagan, 2004). Professional voice users may be particularly susceptible to such fatigue. According to one

report, $80 \%$ of teachers studied experienced vocal fatigue and of those, $22 \%$ experienced it on a daily basis (Gotaas \& Starr, 1993). In another study, 100\% of professional actors evaluated had experienced vocal fatigue and $40 \%$ of actors and $20 \%$ of singers experienced vocal fatigue when performing, specifically (Kitch \& Oates, 1994). In addition to its functionally debilitating nature, individuals with vocal fatigue often have emotional and behavioral reactions to the condition as well [e.g.(McCabe \& Titze, 2002)]. Further, the effect of vocal fatigue on an individual's life is 
commonly underestimated by clinicians and lay people, often increasing the affected individual's level of frustration and even despair (McCabe \& Titze, 2002). In sum, although vocal fatigue is not a life-threatening health condition in itself, its sequelae can be non-trivial [e.g.(Chang \& Karnell, 2004)]. Due to the importance of this phenomenon as a clinical entity, the topic has been the target of increasing research efforts. Unfortunately, the existing body of literature has not yet shed clear light on vocal fatigue’s underlying mechanisms or its effective treatment.

In sharp contrast to the situation for voice, fatigue as a common physical phenomenon more generally has received extensive attention in the physical therapy, sports medicine, and exercise physiology literature. Three broad schools of thought identify mechanisms pertaining to the origins of physical fatigue: (1) peripheral mechanisms (Allen, Lamb, \& Westerblad, 2008; Asmussen, 1979; Edwards, 1981; Fitts, 1994; Westerblad \& Allen, 2002, 2003; Westerblad, Allen, \& Lannergren, 2002; Westerblad, Lee, Lannergren, \& Allen, 1991), (2) central mechanisms (Gandevia, 2001; Gandevia, Enoka, McComas, Stuart, \& Thomas, 1995; Weir, Beck, Cramer, \& Housh, 2006), and (3) and peripheral plus central mechanisms. Different treatment strategies include diet/metabolite replenishment, cardiovascular training and efficiency training.

The next pages and chapters provide pertinent background information on vocal fatigue, fatigue as understood in the exercise physiology domain, and how such information can be adapted to the voice domain. In particular, Chapter 1 will discuss information pertaining to vocal fatigue. Chapter 2 will address fatigue in the exercise physiology domain. Chapter 3 will address the integration of information from exercise physiology and voice domains laying the foundation for present and future research. Research questions and hypotheses will be outlined. Chapter 4 will deal with research design and methodology and data reduction for the proposed 
research. Results from the current study will be presented in Chapter 5 and the document will conclude with discussion and future directions in Chapter 6.

\subsection{DEFINITIONS OF VOCAL FATIGUE}

Vocal fatigue is a term that has been commonly and implicitly used in the clinical literature. Many people, including clinicians and patients seem to know what vocal fatigue is, but have not been able to define it (Hunter \& Titze, 2009). Unfortunately, the term has eluded any precise definition that is convergent across authors (Boucher \& Ayad, 2009; Solomon, 2007; Welham \& Maclagan, 2003). A primary conundrum arises from the question of whether fatigue should be defined in terms of a set of symptoms experienced by an individual subsequent to voice use or in terms of physiological changes that arise as a consequence of vocal work.

Traditionally, in clinical practice, vocal fatigue has been defined in terms of the symptoms a person experiences. According to a published clinical consensus document, clinicians generally tend to define vocal fatigue as a feeling of local tiredness and weak voice after a period of voice use (Verdolini, Rosen, \& Branski, 2006). In addition, clinicians have also described vocal fatigue as a global syndrome identified by a series of symptoms including a patient's perception of increased vocal effort, laryngeal discomfort, neck or shoulder tension, throat or neck pain, reduced pitch range, loss of vocal flexibility, reduced vocal projection or power, reduced vocal control, voice loss, increase in symptoms across the speaking day, and typically, improvement in symptoms with rest (Colton, Casper, \& Leonard, 2006; Gotaas \& Starr, 1993; Kitch \& Oates, 1994; Kostyk \& Putnam Rochet, 1998; Stemple, Stanley, \& Lee, 1995). Vilkman (2004) defined vocal fatigue as a self-perceived condition involving negative 
sensations related to voicing. The self-report approach is appealing considering that vocal fatigue is a complex clinical entity and is variable across individuals. Unfortunately, with this approach, there is no agreed-upon method that reliably and validly identifies individuals with the condition, or to define precisely what it is.

An alternate approach has focused on perceptual, acoustic, or physiologic changes observed as a consequence of prolonged voice use, which is assumed to place a burden on the vocal system thereby inducing fatigue (Boucher \& Ayad, 2009; Scherer, et al., 1991). Under this concept, Scherer et al. (1991) defined vocal fatigue as a "negative vocal adaptation that occurs as a consequence of prolonged voice use.” Such adaptation was construed in perceptual, acoustic, or physiologic terms that would indicate undesirable or unexpected changes in the normal status or function of the laryngeal mechanism. This approach may provide some information about the underlying mechanisms of vocal fatigue. However, circularity is invoked: changes in voice following extended voice use are assumed to reflect vocal fatigue, but vocal fatigue is not defined independent of those changes.

A somewhat convergent approach, taken by McCabe and Titze (2002), considered the problem of vocal fatigue from perspectives described in the physiology and kinesiology literature (Enoka \& Stuart, 1992), according to which fatigue is defined as an acute impairment of performance resulting in both an increased sense of effort and an eventual compromise in force production. Accordingly, McCabe and Titze (2002) defined vocal fatigue as a "progressive increase in perceived phonatory effort accompanied by a progressive decrease in phonatory capabilities.” Finally, Solomon (2007) incorporated the concept of rest in her consideration of vocal fatigue. She proposed that vocal fatigue could be defined as a "perception by the voice 
user, manifested primarily as a sense of increased vocal effort that increases over time with voice use, and subsides with voice rest.”

In physiological terms, vocal fatigue has been classified in terms of both laryngeal muscle fatigue and laryngeal tissue fatigue (McCabe \& Titze, 2002; Titze, 1999). Laryngeal muscular fatigue is potentially caused by a reduction in short-term strength and speed of muscular contraction due to depletion of metabolites and accumulation of by-products. On the other hand, "non-muscular laryngeal tissue fatigue may be caused by temporary changes or damage to the lamina propria (e.g., vibration exposure) from phonation and the excessive damage from such phonation is phonotrauma” (Hunter \& Titze, 2009). Although physiological definitions of fatigue have been suggested in the voice domain, actual physiological mechanisms underlying such fatigue have remained speculative due to the lack of a clear definition of vocal fatigue in the first place (Hunter \& Titze, 2009).

To summarize, based on the existing literature, considerable variability exists across definitions of vocal fatigue and thus far, consensus lacks about how this phenomenon can or should be defined (Solomon, 2007; Welham \& Maclagan, 2003). In short, vocal fatigue can be considered as either a clinical syndrome or as a series of physiological effects from prolonged voice use.

\subsection{EXISTING LITERATURE ON VOCAL FATIGUE}

Different study methodologies have been used to assess vocal fatigue and its associated characteristics. Approaches have included: (a) the study of individuals with and without vocal fatigue complaints; (b) the study of voice changes following prolonged voice use; (c) the study 
of treatment outcomes for vocal fatigue, and (d) study of recovery from vocal loading. Reviews follow and are summarized in Tables 1.1 - 1.4. Of particular interest for present purposes will be the studies' ability to shed light or not on possible mechanisms in vocal fatigue. To our knowledge, thus far studies on vocal fatigue have addressed only adults and some have further specifically addressed the special population of occupational voice users such as teachers and singers.

\subsubsection{Symptoms and signs of vocal fatigue as a function of subject type}

Table 1-1 provides a summary of studies on symptoms and signs of vocal fatigue as a function of subject type. Details are as follows. Gotaas and Starr (1993) investigated factors related to vocal fatigue in a group of teachers, 22 with complaints of vocal fatigue and 17 without complaints (control group). These authors define vocal fatigue as "a problem that begins to occur as the speaking day progresses, is most evident at the end of the day and usually disappears by the following morning." Subjects recorded speech tasks at the beginning (AM) and end (PM) of each teaching day. They rated symptoms of fatigue, harshness, breathiness and pain on a seven-point scale after each AM and PM recording. Eight graduate students in speech pathology served as listeners and used the same scale to rate a set of subject's speech samples presented in randomized order. Results revealed a clear increase in symptoms of vocal fatigue at the end of a working day in teachers with complaints, but not in the control group. 
Table 1-1 Summary of studies on symptoms and signs of vocal fatigue as a function of subject type

\begin{tabular}{|c|c|c|c|c|c|}
\hline $\begin{array}{l}\text { Authors } \\
\text { \& Year }\end{array}$ & Subjects & $\begin{array}{l}\text { Definition } \\
\text { of Fatigue } \\
(\mathrm{Y} / \mathrm{N})\end{array}$ & Protocol & Measures & Findings \\
\hline $\begin{array}{l}\text { Gotaas } \\
\& \text { Starr, } \\
1993\end{array}$ & $\begin{array}{l}\text { Teachers } \\
\text { VF: }=22 \\
(9 \\
\text { males,13 } \\
\text { females), } \\
\text { VH =17 } \\
\text { (6 } \\
\text { males,11 } \\
\text { females) }\end{array}$ & Yes & $\begin{array}{l}\text { VF: AM and } \\
\text { PM speech } \\
\text { recordings } \\
\text { on two } \\
\text { fatigue and } \\
\text { non-fatigue } \\
\text { days } \\
\text { VH: } \\
\text { Reading and } \\
3 \text { min } \\
\text { conversation } \\
\text { on two } \\
\text { separate } \\
\text { days }\end{array}$ & $\begin{array}{l}\text { 1) Participants } \\
\text { self-rated using 7- } \\
\text { pont rating scale } \\
\text { on harshness, } \\
\text { breathiness, strain, } \\
\text { fatigue, tenseness, } \\
\text { loudness, and self- } \\
\text { perceived effort. } \\
\text { 2) Eight graduate } \\
\text { students used same } \\
\text { rating scale to rate } \\
\text { fatigue, harshness, } \\
\text { breathiness, and } \\
\text { strain. }\end{array}$ & $\begin{array}{l}\text { 1) VF group: AM and } \\
\text { PM ratings differed } \\
\text { significantly and AM } \\
\text { ratings on fatigue day } \\
\text { significantly higher than } \\
\text { AM ratings on non- } \\
\text { fatigue day. No } \\
\text { difference for VH group. } \\
\text { 2) Deterioration in } \\
\text { symptoms from AM to } \\
\text { PM on fatigue days for } \\
\text { VF group. Improvement } \\
\text { in symptoms } \\
\text { for both groups on non- } \\
\text { fatigue days }\end{array}$ \\
\hline $\begin{array}{l}\text { Beukers, } \\
1998\end{array}$ & $\begin{array}{l}\mathrm{VF}=20 \\
\text { females } \\
\mathrm{VH}=12 \\
\text { females }\end{array}$ & No & $\begin{array}{l}\text { Voice } \\
\text { endurance } \\
\text { test: reading } \\
\text { each task } \\
\text { (e.g., } \\
\text { reading } \\
\text { aloud, in a } \\
\text { whisper, in } \\
\text { a low, } \\
\text { creaky } \\
\text { voice, make } \\
\text { noises, sing } \\
\text { at different } \\
\text { pitches, } \\
\text { cough three } \\
\text { times) for } 5 \\
\text { min each- } \\
\text { total of } 30 \\
\text { min }\end{array}$ & $\begin{array}{l}\text { 1) Relative } \\
\text { average } \\
\text { perturbation } \\
\text { (RAP), Pitch } \\
\text { period perturbation } \\
\text { quotient (PPQ), } \\
\text { Shimmer, } \\
\text { smoothed } \\
\text { amplitude } \\
\text { perturbation } \\
\text { quotient (sAPQ) } \\
\text { and NHR. } \\
\text { 2) Pain, fatigue, } \\
\text { discomfort, and } \\
\text { globus sensation } \\
\text { evaluated on a } \\
\text { visual analog scale }\end{array}$ & $\begin{array}{l}\text { 1) No changes in any } \\
\text { measures before and after } \\
\text { a working day as well as } \\
\text { on VIT in VF group. No } \\
\text { difference between the } \\
\text { two groups. } \\
\text { 2) No change in ratings } \\
\text { before and after working } \\
\text { days in VF group, but } \\
\text { reports of increase in } \\
\text { symptoms } \\
\text { after the VIT. Fatigue } \\
\text { increased significantly in } \\
\text { VH group after a working } \\
\text { day, and after VIT. }\end{array}$ \\
\hline
\end{tabular}


Table 1-1 (continued)

\begin{tabular}{|c|c|c|c|c|c|}
\hline & & & & $\begin{array}{l}\text { sensation } \\
\text { evaluated on a } \\
\text { visual analog } \\
\text { scale }\end{array}$ & \\
\hline $\begin{array}{l}\text { Laukkanen } \\
\text { et al., } 2006\end{array}$ & $\begin{array}{l}\text { VH- } 79 \\
\text { females }\end{array}$ & No & $\begin{array}{l}\text { AM and PM } \\
\text { recordings of } \\
\text { a prose of } 1 \\
\text { min duration } \\
\text { at } \\
\text { conversational } \\
\text { and loud } \\
\text { levels. Sustain } \\
\text { /a/ at habitual } \\
\text { pitch and } \\
\text { loudness }\end{array}$ & $\begin{array}{l}\text { 1) Fo, Jitter, } \\
\text { Shimmer and } \\
\text { Intensity (SPL), } \\
\text { alpha ratio } \\
\text { 2) Questionnaire } \\
\text { on vocal fatigue } \\
\text { (voice quality, } \\
\text { voice production } \\
\text { and throat } \\
\text { tiredness) on a } \\
\text { visual analog } \\
\text { scale }\end{array}$ & $\begin{array}{l}\text { 1) Increase in Fo, SPL } \\
\text { and alpha ratio; } \\
\text { decrease in Jitter and } \\
\text { Shimmer- post } \\
\text { working day (PM) } \\
\text { 2) Increased rating of } \\
\text { tiredness of throat- } \\
\text { PM }\end{array}$ \\
\hline $\begin{array}{l}\text { Eustace, } \\
\text { Stemple, } \\
\text { and Lee, } \\
1996\end{array}$ & $\begin{array}{l}\text { Retrospective } \\
\text { review, VF = } \\
88 \text { (36 men } \\
\text { and } 52 \\
\text { women) }\end{array}$ & No & $\begin{array}{l}\text { Review of } \\
\text { acoustic, } \\
\text { aerodynamic, } \\
\text { and } \\
\text { stroboscopic } \\
\text { data from } \\
\text { production of } \\
\text { /a/, /i/, /u/ at } \\
\text { low, } \\
\text { comfortable, } \\
\text { and high pitch } \\
\text { levels. }\end{array}$ & $\begin{array}{l}\text { Fo, Jitter, MPT, } \\
\text { Flow volume, } \\
\text { Flow rate, Nine } \\
\text { dimensions of } \\
\text { phonatory } \\
\text { function. }\end{array}$ & $\begin{array}{l}\text { MPT lower than } \\
\text { norms in both males } \\
\text { and females at all } 3 \\
\text { pitch levels. Men } \\
\text { between } 70 \text { and } 79 \\
\text { years had greater flow } \\
\text { ( } 200 \mathrm{ml} / \mathrm{s} \text { ) at high pitch } \\
\text { levels. Different types } \\
\text { of glottal closure } \\
\text { patterns with a } \\
\text { majority presenting } \\
\text { with an abnormal } \\
\text { anterior glottal chink. }\end{array}$ \\
\hline
\end{tabular}

In another study, a voice endurance test, the Voice Interval Test (VIT), was used in the assessment of vocal fatigue in two groups of participants including individuals with and without complaints of vocal fatigue (Table 1-1) (Buekers, 1998). The VIT included a series of fatiguing tasks such as: "read the next page as loudly as possible, read the next page at a whisper, read the next page with a low creaky voice, make a number of noises, sing Frère Jacques three times at a 
different pitch, cough three times, read the next page in a child's voice” etc. The series of fatiguing tasks lasted five minutes in total and was repeated six times without breaks to create an endurance task of 30 minutes. The authors identified these tasks as comparable to the vocal load a teacher would encounter during a typical teaching day. Self-reports on symptoms of voice fatigue (not defined), discomfort, and pain increased following the VIT in the group with vocal fatigue. However, only the symptom "fatigue" increased significantly in the control group. Almost no significant differences were obtained in terms of instrumented acoustic and electroglottographic measures across groups, except for a decreased vocal fold contact rate at $220 \mathrm{~Hz}$ (low pitch condition) following the VIT in both groups. In addition, there was poor correlation between instrumented measures and self-reports.

In contrast, Laukkanen et al. (2006) found that only tiredness of voice, a self-reported symptom among possible vocal sensations, including changes in voice quality increased at the end of the workday in female teachers with complaints of vocal fatigue- defined as changes in voice as a consequence of vocal loading (Table 1-1). In terms of instrumented measures, a significant increase in fundamental frequency $\left(\mathrm{F}_{0}\right)$, sound pressure level (SPL), alpha ratio (defined as the average sound energy distribution along the frequency range and reflecting phonation type) and a decrease in jitter and shimmer were seen at the end of a working day in teachers with complaints of vocal fatigue. Although changes were observed in the instrumented measures, careful inspection of them indicated that the effects could imply either warm-up or fatigue effects. In other words, the increase in $\mathrm{F}_{0}$, SPL, and alpha ratio are normal adaptations to vocal loading and may lead to decreased perturbations as observed (Laukkanen et al., 2006; Orlikoff \& Baken, 1990; Orlikoff \& Kahane, 1991). Moreover, in the study by Laukkanen et al. 
(2006), the correlation between self-reports and instrumented measures was minimal, as occurred in the study by Buekers (1998).

Finally, in a retrospective study by Eustace et al. (1996), acoustic, aerodynamic, and videostroboscopic data were analyzed from individuals with complaints of existing vocal fatigue without visible organic pathology (Table 1-1). With the exception of a high airflow rate and a decreased maximum phonation time, all other instrumented parameters including fundamental frequency, jitter, and frequency range were within normal range. However, videostroboscopic analysis revealed the presence of anterior glottal chink, anterior and posterior glottal chinks, or spindle-shaped closure patterns in $61 \%$ of the subjects at baseline.

In sum, studies on symptoms and signs associated with complaints of vocal fatigue in individuals who complained of the syndrome have yielded mixed results. Some data have indicated that symptoms of vocal fatigue increase with voice use in subjects who report experiencing abnormal vocal fatigue, but again, subjects were identified having vocal fatigue if they indicated they had symptoms that increased with voice use (Gotaas \& Starr, 1993). Thus, the arguments are circular. On the other hand, according to other studies, some symptoms that are believed by clinicians to be associated with vocal fatigue did not necessarily increase over the teaching day and instrumented measures were difficult to interpret. Moreover, existing data in individuals with and without vocal fatigue fail to reveal robust relations across symptombased and instrumented measures of vocal fatigue in subjects who complain of it. Stated differently, thus far, instrumented measures of voice have not reliably covaried with subjective reports of fatigue in individuals who consider it a clinically relevant condition. 


\subsubsection{Experimentally induced fatigue}

Table 1-2 displays findings from studies on experimentally induced vocal fatigue. In this approach, subjects are exposed to a substantial vocal workload and observe subsequent changes in voice functions are observed. The inherent assumption is that any changes seen following exposure to a workload will reflect vocal fatigue. One example is a study by Vintturi and colleagues (2003), who assessed subjective symptoms of vocal fatigue in vocally healthy male and female young students, following a vocal loading task. The goal was to simulate a working day. Participants were asked to read out loud from a novel for $45 \mathrm{~min}$, and repeat the exercise five times over an 8-hour period. Seventeen fatigue symptoms were evaluated, categorized according to five factors including (a) central fatigue (associated with weariness and aversion to work), (b) symptoms affecting neck, shoulders, and back, (c) dry mouth and throat, (d) other throat symptoms (e.g., tired, sore throat, itchy throat) and (e) voice symptoms. Results revealed an increase in throat symptoms and dry mouth and throat following the $3^{\text {rd }}$ or $5^{\text {th }}$ loading sessions. Effects were more marked for females as compared to males. 
Table 1-2 Summary of studies on experimentally induced fatigue

\begin{tabular}{|c|c|c|c|c|c|}
\hline $\begin{array}{l}\text { Authors } \\
\text { \& Year }\end{array}$ & Subjects & $\begin{array}{l}\text { Definition } \\
\text { of Fatigue } \\
(\mathrm{Y} / \mathrm{N})\end{array}$ & Protocol & Measures & Findings \\
\hline $\begin{array}{l}\text { Vintturi, } \\
\text { Alku, } \\
\text { Sala, } \\
\text { Sihvo, \& } \\
\text { Vilkman, } \\
2003\end{array}$ & $\begin{array}{l}\mathrm{VH}=40 \\
\text { men and } \\
40 \\
\text { women }\end{array}$ & Yes & $\begin{array}{l}5 x 45 m i n \text { of } \\
\text { reading a } \\
\text { novel- vocal } \\
\text { loading task. } \\
\text { Had a lunch } \\
\text { break for } \\
45 \text { min and } \\
\text { another } 45 \\
\text { min after the } \\
5^{\text {th }} \text { loading } \\
\text { session-rest. } \\
\text { Subjects were } \\
\text { exposed to } \\
\text { low and high } \\
\text { humidity, soft } \\
\text { and loud } \\
\text { output level } \\
\text { and sitting or } \\
\text { standing } \\
\text { posture. There } \\
\text { were } 5 \text { in each } \\
\text { cell } \\
\text { combination } \\
\text { (gender Vs } \\
\text { every } \\
\text { exposure } \\
\text { group) }\end{array}$ & $\begin{array}{l}17 \text { questions } \\
\text { related to } \\
\text { symptoms } \\
\text { factored into } 5 \\
\text { factors including } \\
\text { a) central } \\
\text { fatigue, b) } \\
\text { shoulder, neck, } \\
\text { and back, c) } \\
\text { drying in the } \\
\text { mouth and } \\
\text { throat, d) } \\
\text { symptoms of the } \\
\text { throat, and e) } \\
\text { symptoms of } \\
\text { voice }\end{array}$ & $\begin{array}{l}\text { Women had more } \\
\text { loading related } \\
\text { symptoms than men. } \\
\text { Women had more } \\
\text { problems with humidity } \\
\text { than men. } \\
\text { Standing posture had } \\
\text { more issues than sitting. }\end{array}$ \\
\hline $\begin{array}{l}\text { Welham } \\
\& \\
\text { Maclagan, } \\
2004\end{array}$ & $\begin{array}{l}5 \text { trained } \\
\text { signers, } \\
2 \text { had } \\
\text { voice } \\
\text { problems }\end{array}$ & No & $\begin{array}{l}\text { Vocal warm- } \\
\text { up (10 min) } \\
\text { followed by } \\
40 \text { min of } \\
\text { simulated solo } \\
\text { performance, } \\
\text { and } 10 \text { min of } \\
\text { vocal rest }\end{array}$ & $\begin{array}{l}\text { 1) } \mathrm{F}_{0} \text { in the final } \\
\text { /i/ of three, } \\
\text { maximum and } \\
\text { minimum } \\
\text { attainable pitch, } \\
\text { SPL (max) } \\
\text { attained during } \\
\text { sustained } \\
\text { phonation of /a/. } \\
\text { 2) Self-report } \\
\text { data collected on } \\
\text { the following } \\
\text { day. }\end{array}$ & $\begin{array}{l}\text { 1) No change in } \\
\text { parameters across warm- } \\
\text { up and performance } \\
\text { periods } \\
\text { RAP, APQ, HNR were } \\
\text { variable } \\
\text { 2) } 3 \text { out of } 5 \text { experienced } \\
\text { fatigue and } 3 / 5 \text { also } \\
\text { reported a decrease in } \\
\text { fatigue following rest. }\end{array}$ \\
\hline
\end{tabular}


Table 1-2 (continued)

\begin{tabular}{|c|c|c|c|c|c|}
\hline $\begin{array}{l}\text { Stemple et } \\
\text { al., } 1995\end{array}$ & $\begin{array}{l}\text { VH-10 } \\
\text { females }\end{array}$ & Yes & $\begin{array}{l}\text { Reading at } 75- \\
80 \mathrm{~dB} \text { for } 2 \\
\text { hours with a } 5 \\
\text { min break after } \\
1 \text { hour }\end{array}$ & $\begin{array}{l}\text { Pre and post } \\
\text { loading task: } \\
\text { 1) Acoustic } \\
\text { measures- } F_{0} \text {, } \\
\text { Jitter, and } \\
\text { frequency range } \\
\text { 2) Aerodynamic } \\
\text { measures- MPT, } \\
\text { phonation volume } \\
\text { and flow rate for } \\
\text { /a, i, u/ at } 2 \\
\text { different pitch } \\
\text { levels } \\
\text { 3) } \\
\text { Videostroboscopic } \\
\text { evaluation } \\
\text { 4) Self reports of } \\
\text { effort }\end{array}$ & $\begin{array}{l}\text { Post-test: } \\
\text { 1) Increased mean } F_{0} \text {; } \\
\text { lower mean Jitter. } \\
\text { 2) No difference in other } \\
\text { acoustic and } \\
\text { aerodynamic measures } \\
\text { 3) Videostroboscopic } \\
\text { evaluation: appearance } \\
\text { of chinks and abnormal } \\
\text { glottal closure (9/10) } \\
\text { 4) } 100 \% \text { maximum } \\
\text { effort at the end of } 2 \\
\text { hours of reading in } 8 / 10\end{array}$ \\
\hline $\begin{array}{l}\text { Boucher et } \\
\text { al., } 2006\end{array}$ & $\begin{array}{l}\mathrm{VH}, 5 \\
\text { men } \\
\text { and } 2 \\
\text { women }\end{array}$ & No & $\begin{array}{l}\text { 12-14 hr } \\
\text { protocol with } 2 \\
\text { breaks to eat. } \\
\text { Every } 12-15 \\
\text { min, } 2 \text { tasks: } \\
\text { a) vocal effort } \\
\text { task- reading } \\
\text { aloud at } 74 \mathrm{~dB} \\
\text { for } 3 \text { min } \\
\text { b) vocalization } \\
\text { task- six tokens } \\
\text { of /a/ at modal } \\
\text { and high pitch }\end{array}$ & $\begin{array}{l}\text { 1) EMG spectral } \\
\text { compression }\end{array}$ & $\begin{array}{l}\text { 1) EMG spectral } \\
\text { compression was } \\
\text { variable, but was } \\
\text { observed at the end of } \\
\text { the test period for all } \\
\text { participants, indicating } \\
\text { fatigue with effortful } \\
\text { voicing task. }\end{array}$ \\
\hline $\begin{array}{l}\text { Boucher \& } \\
\text { Ayad, } \\
2009\end{array}$ & $\begin{array}{l}\text { VH, } 5 \\
\text { men } \\
\text { and } 2 \\
\text { women }\end{array}$ & No & $\begin{array}{l}\text { 12-14hr } \\
\text { protocol with } 2 \\
\text { breaks to eat, } \\
\text { with and } \\
\text { without vocal } \\
\text { loading task. } \\
\text { Only } \\
\text { vocalization } \\
\text { task at modal } \\
\text { pitch every } 12- \\
15 \text { min }\end{array}$ & $\begin{array}{l}\text { 1) } \mathrm{F}_{0} \text {, intensity, } \\
\text { frequency of } \\
\text { amplitude tremor } \\
\text { (FATr), and } \mathrm{F}_{0} \\
\text { tremor (FFTr) } \\
\text { compared with } \\
\text { and without vocal } \\
\text { loading task }\end{array}$ & $\begin{array}{l}\text { 1) No change in } F_{0} \text {, } \\
\text { intensity or FFTr with } \\
\text { vocal loading. } \\
\text { Consistent changes in } \\
\text { FATr with vocal } \\
\text { loading- indicator of } \\
\text { vocal effort }\end{array}$ \\
\hline
\end{tabular}


Table 1-2 (continued)

\begin{tabular}{|c|c|c|c|c|c|}
\hline $\begin{array}{l}\text { Chang and } \\
\text { Karnell, } \\
2004\end{array}$ & $\begin{array}{l}\mathrm{VH}, 5 \\
\text { men } \\
\text { and } 5 \\
\text { women }\end{array}$ & No & $\begin{array}{l}\text { Reading for tw } \\
\text { hours at } 75-85 \\
\text { dB. Measures } \\
\text { were every } 30 \\
\text { min during } \\
\text { fatiguing task } \\
\text { and } 15 \mathrm{~min}, 1 \\
\mathrm{hr}, 2 \mathrm{hr}, 24 \mathrm{hr} \\
\text { and } 72 \mathrm{hr} \text { post } \\
\text { loading }\end{array}$ & $\begin{array}{l}\text { 1) PTP measured } \\
\text { during CVC } \\
\text { repetitions at } 3 \\
\text { different pitch } \\
\text { levels. } \\
\text { 2) PPE was used } \\
\text { to correlate with } \\
\text { PTP }\end{array}$ & $\begin{array}{l}\text { 1) PTP increased during } \\
\text { the fatiguing task } \\
\text { compared to baseline at } \\
\text { all three pitch levels, but } \\
\text { significantly higher for } \\
\text { the high pitch condition. } \\
\text { PPE correlated well with } \\
\text { PTP at low and } \\
\text { comfortable conditions } \\
\text { only. There is no effect } \\
\text { of gender. }\end{array}$ \\
\hline
\end{tabular}

In difference to the foregoing study, most studies using the vocal loading approach have used both self-report and instrumented measures. For example, Stemple et al. (1995) reported on 10 vocally healthy female subjects who were exposed to a 2-hr vocal loading task. Most participants reported increased effort to produce voice following loading. Instrumented findings revealed a significant decrease in jitter and an increase in mean speaking fundamental frequency $\left(\mathrm{F}_{0}\right)$, and the appearance of an anterior glottal chink in 6 of the 10 participants, following the task. Although changes in jitter and $\mathrm{F}_{0}$ were considered possible indicators of "fatigue," they could as easily be attributed to "warm up" effects more readily than to fatigue (Orlikoff \& Baken, 1990). Interestingly, no sign of any correlation was detected between self-report and instrumented measures of fatigue, as for other studies (Beukers, 1998; Laukkanen et al., 2006).

Another group assessed five vocally healthy trained singers, using self-report and acoustic measures after warm-up, during and after a solo vocal performance and after rest. Results for self-report measures were highly variable across participants, and again the correspondence between self-report and instrumented measures was poor (Welham \& Maclagan, 
2004). One very plausible hypothesis to entertain is that in this case and in others, variability in results across individuals reflects variable susceptibility to fatigue itself.

A few studies using the vocal loading approach have only assessed changes in instrumented measures of voice. Gelfer et al. (1991) exposed trained and untrained vocally healthy singers to a reading task for one hour at $80 \%$ of each subject's individual maximum intensity. Acoustic measures including intensity, $\mathrm{F}_{0}$, jitter ratio, shimmer, and signal-to-noise ratio were obtained before and after the task. With the exception of a decreased jitter ratio, the trained singers did not show any differences in measures pre- versus post-loading. However, untrained singers demonstrated an increase in fundamental frequency, intensity, and signal-tonoise ratio after loading. As for results reported by Laukkanen et al. (2006) and Stemple et al. (1995), $\mathrm{F}_{0}$, signal-to-noise ratio and intensity changes could be argued to be either warm-up or fatigue effects (Orlikoff \& Baken, 1990; Orlikoff \& Kanache, 1991).

More recently, Boucher and colleagues (Boucher, Ahmarani, \& Ayad, 2006) subjected seven subjects to a vocal loading task involving reading loudly for three minutes every 12-15 minutes for 12-14 hr. Hooked wire electrodes were inserted into the lateral cricoarytenoid muscle bilaterally and electromyographic (EMG) signals were captured at regular intervals during the entire exposure period and during the vocalization of /a:/ at modal and at high pitches after each vocal loading task. Results indicated spectral compression with prolonged reading over the course of the day indicating neuromuscular fatigue. However, the authors did not have the means to distinguish between general fatigue over the course of the day versus vocal fatigue specific to the loading task. In addition to EMG measures, the authors also attempted to estimate acoustic correlates of laryngeal fatigue. No consistent correlations were observed between acoustic 
measures and estimates of muscle fatigue, with the exception of a connection between vocal tremor and muscle fatigue as observed by EMG (Boucher \& Ayad, 2009).

Summarizing the literature to this point, including literature on subjects with declared vocal fatigue and literature involving experimentally induced vocal fatigue, the following patterns emerge: (1) self-reported symptoms of vocal fatigue tend to be greater in individuals who complain of the syndrome, and tend to increase with vocal loading even in healthy subjects; (2) $\mathrm{F}_{0}$ and possibly intensity tend to increase after a period of vocal loading, both in individuals with complaints of vocal fatigue and those without it; (3) an anterior glottal chink is found in many individuals who self-identify as having the fatigue syndrome and in several healthy individuals who have been exposed to a loading task; and (4) spectral compression in the laryngeal EMG signal and possibly tremor may be indicators of neuromuscular vocal fatigue following vocal loading in vocally healthy individuals. However, an equally evident pattern is that (5) self-report and instrumented measures have generally failed to covary in vocal fatigue studies. Possibly one of the reasons is that attempts were not made in the relevant studies to assess self-report and instrumented measures that should covary, even conceptually.

Chang and Karnell (2004) attempted to address this shortcoming by using a vocal loading task to evaluate covariations across a perceptual measure of vocal fatigue - perceived phonatory effort (PPE) - and an instrumented measure of phonatory effort - phonation threshold pressure (PTP). In brief, PTP reflects the minimal respiratory effort required to initiate and sustain vocal fold oscillation (Titze, 1988), and has been estimated to account for about 50\% of the variance in PPE at least in trained vocalists (Colton, personal communication with Verdolini Abbott). Five men and five women were subjected to a prolonged reading task that involved reading aloud for two hours at 75-85 dB SPL measured at 18 inches from the speaker's mouth. PTP at low, mid, 
and high pitches and PPE measures were obtained prior to, during and following the vocal loading task at 15 different time points. Both PPE and PTP increased steadily during the vocal loading task. Within 15 minutes of completion of the loading task, PTP returned to baseline for the high-pitch condition. However, comfortable and low-pitched PTP were more resistant returning to baseline at the one-hour follow-up time point. In contrast, for PPE, participants required about 2 hours to recover totally from the loading task. Correlation coefficients using the Fisher z transformation indicated a good correlation between mean PTP and mean ratings of PPE at the low and comfortable pitches, but this correlation was not observed at high pitches.

In sum, across the literature reviewed thus far, findings implicating possible physiological mechanisms in vocal fatigue are sparse, at best. Further, self-report and instrumented measures of possible fatigue have failed to reveal connections.

\subsubsection{Treatment of vocal fatigue}

Extremely few studies have targeted treatment paradigms or approaches that may prevent or treat vocal fatigue. Findings are summarized in Table 1-3. McCabe and Titze (McCabe \& Titze, 2002), reported on four teachers with a history of vocal fatigue. All participants received six onehour sessions of chant therapy for 2 weeks and six one-hour sessions of Physiologic Recovery Treatment (placebo therapy) for 2 weeks. Two participants received chant therapy first followed by placebo therapy and the order was reversed for the other two participants. Participants were presented with both therapies as a viable treatment option "to determine the differential contributions of organic and functional components to the propagation of vocal fatigue symptoms.” Prior to and following completion of each of the interventions, a vocally fatiguing task was administered, consisting of a 2-hr period of loud reading loud in a large classroom. Self- 
ratings of vocal effort and voice quality were obtained $(0$ = "no vocal effort" and "best sounding voice;" and 10 = "maximum vocal effort imaginable" and "worst sounding voice imaginable") prior to the loading task, every 15 minutes during the task, at 15-min intervals following task completion (up to one hour), and four times daily until ratings returned to baseline. Ratings were later plotted to obtain fatigue response profiles (FRP). Results indicated that mean effort ratings decreased following chant therapy, compared to pre therapy (pre-treatment 8.86 and posttreatment 4.26). Also voice quality ratings improved following chant therapy (pre-treatment 6.40 and post-treatment 3.33). In contrast, improvements in average ratings were minimal following the placebo therapy.

Results from other treatment studies on the benefits of interventions for vocal fatigue, specifically vocal warm-up and hydration interventions for vocal fatigue, have been more equivocal (Table 1-3). Milbrath and Solomon (2003) assessed the effects of vocal warm-ups (respiratory, resonance and phonatory exercises) as compared to effects of a vocal rest "placebo" on the outcome of a vocal loading task. Subjects were eight young women who reported symptoms of chronic vocal fatigue. The two experimental conditions were alternated between participants. The second experimental condition was separated from the first by a $72 \mathrm{hr}$ period to allow for full recovery from initial loading task. The loading task itself involved one hour of prolonged loud reading, followed by 30 min of silence. PTP and PPE were measured prior to, during, and following the loading task. Somewhat interestingly, predictions that warm-ups would decrease both PTP and PPE compared to rest post loading was not observed. Results failed to reveal benefits from the warm-ups for the alleviation of putative vocal fatigue.

In a somewhat similar vein, Solomon and colleagues (2000) used a within-subject design to investigate the effects of systemic hydration on PTP and PPE in four vocally healthy untrained 
women before and after a two-hour prolonged reading task. PTP and PPE measurements were made before, during and after the loading task for both experimental conditions (low hydration $16 \mathrm{oz}$ water only per day and high hydration- five $16 \mathrm{oz}$ bottles of water per day for 2 days before loading). Experimental conditions were separated by 3 days. Three of the four women showed a delay in the increase of PTP with high hydration condition prior to the start of the loading task. In a second study, the effects of typical, low and high hydration on PTP and PPE administering the same loading and data collection protocol were investigated in four vocally healthy men. Only two of four men showed benefits under the high hydration condition compared to low hydration condition (Solomon, Glaze, Arnold, \& van Mersbergen, 2003). 
Table 1-3 Summary of Treatment Studies in Vocal Fatigue

\begin{tabular}{|c|c|c|c|c|c|}
\hline $\begin{array}{l}\text { Authors } \\
\text { and Year }\end{array}$ & Subjects & $\begin{array}{l}\text { Definition } \\
\text { of fatigue } \\
(\mathrm{Y} / \mathrm{N})\end{array}$ & Protocol & Measures & Findings \\
\hline $\begin{array}{l}\text { McCabe } \\
\text { and Titze, } \\
2002\end{array}$ & $\begin{array}{l}\text { Teachers } \\
\text { VF }=4 \\
(3 \\
\text { females } \\
\text { and } 1 \\
\text { male) }\end{array}$ & Yes & $\begin{array}{l}\text { Chant therapy- } \\
\text { using a psalm tone. } \\
\text { A psalm tone is a } \\
\text { traditional pattern } \\
\text { of notes to which } \\
\text { any text can be set. } \\
\text { Placebo therapy- } \\
\text { Physiologic } \\
\text { recovery } \\
\text { treatment.- non- } \\
\text { effortful speech. } \\
\text { Subjects underwent } \\
\text { six 1-hr sessions of } \\
\text { both therapies in } \\
\text { two- 2week blocks. } \\
\text { Fatiguing task (pre } \\
\text { and post chant and } \\
\text { placebo therapy)- } \\
\text { 2-hr reading in a } \\
\text { large classroom. }\end{array}$ & $\begin{array}{l}\text { Voice Range } \\
\text { Profile (pre } \\
\text { and post)- to } \\
\text { plot intensity } \\
\text { range vs } \text { F}_{0} \text {. } \\
\text { Self-ratings: } \\
\text { vocal effort } \\
\text { and voice } \\
\text { quality } \\
\text { (before } \\
\text { fatiguing task, } \\
\text { every } 15 \text { min } \\
\text { for one hour } \\
\text { after the task, } \\
\text { four times per } \\
\text { day until } \\
\text { ratings } \\
\text { returned to } \\
\text { BL. } \\
\text { Plot of fatigue } \\
\text { response } \\
\text { profile (FRP). }\end{array}$ & $\begin{array}{l}\text { With chant therapy, } \\
\text { mean effort and } \\
\text { quality ratings } \\
\text { decreased from } 8.68 \\
\text { and } 6.40 \text { to } 4.26 \text { and } \\
3.33 \text { respectively. } \\
\text { Minimal change } \\
\text { following placebo } \\
\text { therapy. } \\
\text { FRP's were a good } \\
\text { indicator of recovery } \\
\text { from VF. }\end{array}$ \\
\hline $\begin{array}{l}\text { Solomon } \\
\text { and } \\
\text { DiMattia, } \\
2000\end{array}$ & $\begin{array}{l}\mathrm{VH}=4 \\
\text { females }\end{array}$ & No & $\begin{array}{l}\text { Effect of systemic } \\
\text { hydration on } \\
\text { laryngeal function } \\
\text { following vocal } \\
\text { loading. Measures } \\
\text { under two } \\
\text { hydration } \\
\text { conditions: low } \\
\text { (one } 16 \text { oz water } \\
\text { per day and no } \\
\text { other beverages for } \\
2 \text { days prior to } \\
\text { protocol) and high ( } \\
5 \text { - } 16 \text { oz }\end{array}$ & $\begin{array}{l}\text { 1) PTP (at } 4 \\
\text { pitches), } \\
\text { 2) PPE- } \\
\text { obtained at } \\
\text { baseline, } \\
\text { following } \\
\text { comfortable } \\
\text { reading } \\
\text { (warm-up), } \\
\text { after one hour } \\
\text { of loud } \\
\text { reading and } \\
\text { after } 2 \text { hours } \\
\text { of loud } \\
\text { reading (2 hrs } \\
\text { of vocal }\end{array}$ & $\begin{array}{l}\text { 1) PTP increased at } 1 \\
\text { hr and at } 2 \text { hrs of } \\
\text { loud reading at high } \\
\text { pitch, specifically } \\
\text { more at low } \\
\text { hydration condition } \\
\text { than high hydration } \\
\text { condition. } 3 \text { subjects } \\
\text { showed greater PTP } \\
\text { at } 1 \text { hour loud } \\
\text { reading for low } \\
\text { hydration condition, } \\
\text { but at } 2 \text { hrs for } 2 \text { of } \\
\text { those subjects at high } \\
\text { hydration condition. } \\
\text { PTP returned to BL }\end{array}$ \\
\hline
\end{tabular}


Table 1-3 (continued)

\begin{tabular}{|c|c|c|c|c|c|}
\hline & & & $\begin{array}{l}\text { bottles plus any } \\
\text { other beverages } 2 \\
\text { days prior to } \\
\text { protocol). } 3 \text { day } \\
\text { interval between } 2 \\
\text { experimental } \\
\text { conditions. }\end{array}$ & $\begin{array}{l}\text { loading), and } \\
\text { following } 15 \\
\text { min of vocal } \\
\text { silence (vocal } \\
\text { recovery). } \\
\text { 3) Laryngeal } \\
\text { images were } \\
\text { obtained at the } \\
\text { same time } \\
\text { points }\end{array}$ & $\begin{array}{l}\text { after 15min of vocal } \\
\text { silence. PTP good } \\
\text { measure, but } \\
\text { systemic hydration } \\
\text { did not have much } \\
\text { impact on reducing } \\
\text { laryngeal function } \\
\text { changes with } \\
\text { prolonged reading. } \\
\text { 2) PPE increased } \\
\text { with loud reading and } \\
\text { decreased with vocal } \\
\text { silence. } \\
\text { 3) Laryngeal images } \\
\text { revealed a spindle } \\
\text { pattern following } \\
\text { prolonged reading } \\
\text { (2/4). }\end{array}$ \\
\hline \begin{tabular}{|l} 
Solomon, \\
Glaze, \\
Arnold, \& \\
Merbergen, \\
2002
\end{tabular} & $\begin{array}{l}\mathrm{VH}=4 \\
\text { males }\end{array}$ & No & $\begin{array}{l}\text { Effect of vocal } \\
\text { loading and } \\
\text { hydration on vocal } \\
\text { function. } \\
\text { Investigated under } \\
3 \text { hydration } \\
\text { conditions" } \\
\text { Typical hydration, } \\
\text { low hydration } \\
\text { (25\% of typical } \\
\text { drinking) and high } \\
\text { hydration (excess } \\
75 \% \text { of typical } \\
\text { drinking). During } \\
\text { prolonged loud } \\
\text { reading (vocal } \\
\text { loading), every } 5 \\
\text { min drank water } \\
\text { for high hydration } \\
\text { condition and dry } \\
\text { swallow for low } \\
\text { hydration } \\
\text { condition. }\end{array}$ & $\begin{array}{l}\text { 1) PTP (at } 4 \\
\text { pitches), } \\
\text { 2) PPE- } \\
\text { obtained at } \\
\text { baseline, } \\
\text { following } \\
\text { comfortable } \\
\text { reading (warm- } \\
\text { up), after one } \\
\text { hour of loud } \\
\text { reading and } \\
\text { after } 2 \text { hours of } \\
\text { loud reading (2 } \\
\text { hrs of vocal } \\
\text { loading), and } \\
\text { following 15 } \\
\text { min of vocal } \\
\text { silence (vocal } \\
\text { recovery). 3) } \\
\text { Laryngeal } \\
\text { images were } \\
\text { obtained before } \\
\text { and after } 2 \\
\text { hours of loud }\end{array}$ & $\begin{array}{l}\text { 1) PTP increased } \\
\text { after } 1 \text { hour (1 to } 2.5 \\
\left.\mathrm{~cm} \mathrm{H}_{2} \mathrm{O}\right) \text { and } 2 \text { hours } \\
\left(1.8 \text { to } 3.7 \mathrm{~cm} \mathrm{H}_{2} \mathrm{O}\right) \\
\text { of loud reading, } \\
\text { specifically at high } \\
\text { pitch. With respect to } \\
\text { effects of hydration, } \\
2 \text { men showed a } \\
\text { delay or attenuation } \\
\text { in PTP following } \\
\text { loud reading in well- } \\
\text { hydrated condition, } \\
\text { whereas } 2 \text { men } \\
\text { showed the opposite } \\
\text { trend. } \\
\text { 2) PTP and PPE was } \\
\text { correlated. } \\
\text { 3) } 2 \text { men had an } \\
\text { anterior glottal gap } \\
\text { following } 2 \text { hours of } \\
\text { loud reading. }\end{array}$ \\
\hline
\end{tabular}


Table 1-3 (continued)

\begin{tabular}{|c|c|c|c|c|c|}
\hline & & & & reading & \\
\hline $\begin{array}{l}\text { Milbrath } \\
\text { and } \\
\text { Solomon, } \\
2003\end{array}$ & $\begin{array}{l}\mathrm{VF}=8 \\
\text { females }\end{array}$ & $\begin{array}{l}\text { Indirectly } \\
\text { defined }\end{array}$ & $\begin{array}{l}\text { Comparison of } \\
\text { effects of vocal } \\
\text { warm-up and } \\
\text { vocal rest prior to } \\
\text { vocal loading } \\
\text { task. } \\
\text { Warm-up } \rightarrow 1 \\
\text { hour loud } \\
\text { reading } \rightarrow 30 \mathrm{~min} \\
\text { vocal silence } \\
\text { Vocal rest } \rightarrow 1 \\
\text { hour loud } \\
\text { reading } \rightarrow 30 \mathrm{~min} \\
\text { vocal silence }\end{array}$ & $\begin{array}{l}\text { 1) PTP (using } \\
\text { /pi/ at } 3 \\
\text { pitches) } \\
\text { 2) PPE. } \\
\text { Measures } \\
\text { taken before } \\
\text { and after each } \\
\text { task and also } \\
\text { intermediate } \\
\text { measures at } \\
\text { 30min interval } \\
\text { for loud } \\
\text { reading and at } \\
\text { 15min interval }\end{array}$ & $\begin{array}{l}\text { 1) PTP increased at } \\
\text { high pitch condition } \\
\text { only following vocal } \\
\text { warm-up. } \\
\text { 2) No difference in } \\
\text { PPE with arm-up or } \\
\text { voice rest post } \\
\text { loading }\end{array}$ \\
\hline
\end{tabular}

Summarizing across treatment studies, to date few if any data are available that shed light on possible mechanisms in vocal fatigue or its effective treatment. This gap in the literature is unfortunate given what is perceived to be the high prevalence of vocal fatigue syndromes and their frequent debilitating nature.

\subsubsection{Recovery from vocal loading}

Finally, some data have been reported regarding characteristics of recovery from presumed vocal fatigue. Results are displayed in Table 1-4. Specifically, Hunter and Titze (2009) attempted to quantify vocal recovery in 86 school teachers. Participants were pre-screened for vocal health using laryngeal stroboscopic evaluation, vocal questionnaires (e.g., Vocal Handicap Index-30; Jacobson et al., 1997 and Vocal Health Questionnaire; adapted from Sapir, Mather-Schmidt, \& Larson, 1996) and an extensive acoustic analysis (not otherwise specified) and were deemed vocally healthy. Following screening, participants were subjected to a 2-hr vocal loading task. 
Self-rating measures on effort level (EFFT), discomfort (DISC), and inability to produce soft voice (IPSV) were obtained prior to, 15 min and every 2 hours following the vocal loading task for 3 days (recovery day 1, 2 and 3) during waking hours. Recovery trajectory was plotted using EFFT, DISC, and IPSV data. Results indicated that on average $50 \%$ of the recovery was achieved within 4-6 hours and 90\% recovery occurred with 12-18 hours post loading. IPSV recovery was the slowest. Results also suggested that the post-loading vocal recovery trajectory paralleled the trajectory reported for chronic dermal wound healing, suggesting that for many individuals vocal damage is ongoing and the healing mechanism is engaged in a state of constant repair. However, the study did not attempt to account for confounds between laryngeal muscular and laryngeal tissue (lamina propria) fatigue.

Table 1-4 Summary of study from vocal loading

\begin{tabular}{|c|c|c|c|c|c|}
\hline $\begin{array}{l}\text { Authors \& } \\
\text { year }\end{array}$ & Subjects & $\begin{array}{l}\text { Definition } \\
\text { of fatigue }\end{array}$ & Protocol & Measures & Findings \\
\hline $\begin{array}{l}\text { Hunter \& } \\
\text { Titze } \\
\text { (2009) }\end{array}$ & $\begin{array}{l}\text { Teachers } \\
\mathrm{N}=87(21 \\
\text { males and } 66 \\
\text { females }\end{array}$ & Yes & $\begin{array}{l}\text { Screening } \\
\text { Vocal loading } \\
\text { task : } 2 \text { hr } \\
\text { reading out } \\
\text { loud- loudness } \\
\text { comparable } \\
\text { with teaching } \\
\text { loudness }\end{array}$ & $\begin{array}{l}\text { Current effort } \\
\text { level (EFFT), } \\
\text { Inability to } \\
\text { produce soft } \\
\text { voice (IPSV), } \\
\text { and laryngeal } \\
\text { discomfort } \\
\text { (DISC)- prior } \\
\text { to vocal } \\
\text { loading, } \\
\text { during } \\
\text { loading, every } \\
2 \text { hrs post } \\
\text { vocal loading } \\
\text { for } 3 \text { days }\end{array}$ & $\begin{array}{l}50 \% \text { of } \\
\text { recovery on } \\
\text { these ratings } \\
\text { achieved } \\
\text { within } 4-6 \\
\text { hrs. } \\
90 \% \text { recovery } \\
\text { within 12-18 } \\
\text { hrs post } \\
\text { loading } \\
\text { Recovery } \\
\text { trajectory was } \\
\text { close to } \\
\text { dermal } \\
\text { healing } \\
\text { trajectory }\end{array}$ \\
\hline
\end{tabular}




\subsection{GAPS IN THE LITERATURE}

Unfortunately, existing literature on vocal fatigue fails to shed any clear light on possible physiological mechanisms underlying vocal fatigue or effective treatment options for it. Mixed, inconclusive results can be attributed to variations in subject population across studies, the use of varying instrumented and self-report measures, and the use of different loading tasks. Specifically, most studies in the vocal fatigue domain have looked at individuals without vocal fatigue and attempts were made to induce fatigue by employing prolonged reading tasks at increased loudness. The implicit assumption is that such tasks would place a load on the vocal system and thereby result in vocal fatigue. However, some individuals without vocal fatigue may be more resistant to vocal fatigue than others, even under those conditions, complicating the interpretation of the data (e.g., Solomon, 2007). Second, the specific set of instrumented measures that have been used, including acoustic, aerodynamic, and visual measures, may not have been sufficiently sensitive or even conceptually appropriate to capture effects of fatigue or underlying mechanisms. In addition, studies explicitly designed to investigate mechanisms underlying fatigue, including peripheral and central mechanisms, are grossly lacking. Finally, the noted lack of a universal definition of vocal fatigue in the literature obscures the issues altogether. In other words, if one wishes to study subjects with chronic vocal fatigue, the lack of a consensus definition of this phenomenon makes it virtually impossible to recruit appropriate individuals.

In sum, existing literature on vocal fatigue has thus far failed to yield insight into possible mechanisms underlying fatigue or its treatment. However, results from existing literature does pave the way for future research in this domain providing insight in to the direction of research (e.g., define vocal fatigue, identify appropriate and sensitive measures to study mechanisms of 
vocal fatigue, etc). In a next generation, a first step towards understanding vocal fatigue will be to first define it and based on that definition, identify a framework within which to study it. Further, in addition to defining fatigue, it will be important to identify valid and reliable measures to study it. Both of these issues are addressed in Chapter 3.

However, first, in the next chapter, we will turn to an overview of fatigue as understood and studies in the exercise physiology domain. Fatigue has been well documented in that domain and underlying mechanisms have been identified. Also various methods have been developed to both assess fatigue and treat it. Information from exercise physiology will be extrapolated to the case of voice, as outlined in Chapter 3. 


\subsection{FATIGUE IN THE EXERCISE PHYSIOLOGY DOMAIN}

The phenomenon of fatigue has been frequently investigated in the exercise physiology domain, given the nature of its importance. Studies have addressed underlying mechanisms according to different schools of thought, including thought directed to peripheral fatigue, central fatigue, or an integration of peripheral and central fatigue. The next pages provide a review.

\subsection{DEFINITIONS OF FATIGUE}

In the exercise physiology domain, muscle fatigue has been defined in different ways. Traditionally, such fatigue has been defined in terms of decrements in performance or force output following an activity (Asmussen, 1979; Brooks, Fahey, \& Baldwin, 2005; Edwards, 1981; Enoka \& Stuart, 1992; Fitts, 1994). In a slightly different approach, fatigue has been defined as an exercise-induced reduction in the ability to exert muscle force or power, regardless of the task sustainability (Bigland-Ritchie \& Woods, 1984; Vollestad, 1997). A third approach has been to acknowledge the influence of increased perceived effort in exerting or sustaining a desired force (Enoka \& Stuart, 1992). Additionally, the onset of fatigue is defined by an increase in levels of oxygen uptake during an exercise (Poole, et al., 1991; Ratkevicius, Stasiulis, Dubininkaite, \& Skurvydas, 2006; Reybrouck, Vangesselen, Mertens, \& Gewillig, 2007). This definition will be of particular relevance to the present study and will be discussed under section 2.4.1. 


\subsection{CAUSES OF FATIGUE}

Summarizing research over a span of many years, it is clear that various causes have been suggested; identification of a specific cause has been elusive. According to the school of thought that emphasizes peripheral factors, in which fatigue is defined as a decrease in the ability of a skeletal muscle to generate force following sustained physical activity (St Clair Gibson \& Noakes, 2004), findings have pointed to the working muscles as the site of fatigue. Numerous processes have been implicated including problems with metabolite accumulation (Adams, Fisher, \& Meyer, 1991; Astrand et al., 2003, Bangsbo \& Juel, 2006; Lamb \& Stephenson, 2006; Lannergren \& Westerblad, 1991), substrate/metabolite depletion (Bigland-Ritchie, Cafarelli, \& Vollestad, 1986; Fitts, 1994), excitation-contraction coupling (Allen, Lamb, \& Westerblad, 2008; Dahlstedt \& Westerblad, 2001, and inadequate oxygen supply to the working muscles (Brooks et al., 2005; Degens, Horneros, \& Hopman, 2006; Gonzalez-Alonso \& Calbet, 2003; Hogan, Kohin, Stary, \& Hepple, 1999; Paul \& Wood, 2002). In brief, according to this approach the strong suggestion is that fatigue is a consequence of changes at the level of the working muscle per se, distal to the central nervous system.

According to a complementary if not contrasting approach, the focus is instead on central mechanisms in fatigue. In this approach, fatigue is seen as a reduction in neural drive to the muscle, resulting in a decline in force production, independent of changes in the contractile machinery (Amann \& Calbet, 2008; Enoka \& Stuart, 1992). Central fatigue is attributed to the reduction in neural drive to motor neurons and an inhibition of motoneuron excitability - where a motor neuron is defined as a single motor neuron and all the muscle fibers it innervates subsequent to afferent feedback from the muscle (Davis \& Bailey, 1997; Gandevia, et al., 1995).

A speculation is that such inhibition is the body's attempt to maintain system homeostasis and 
prevent organ (i.e., muscle damage or heart) failure (Lambert, et al., 2005; Noakes, St Clair Gibson, \& Lambert, 2004, 2005; St Clair Gibson \& Noakes, 2004). Additionally, decreased recruitment of many muscle groups simultaneously prevents the progression of peripheral fatigue (Gandevia, 1995). An important point is that a relationship has been identified between metabolite accumulation in the periphery and inhibitory feedback and oxygen supply to the brain to maintain homeostasis, thereby connecting peripheral and central mechanisms in fatigue (Amann \& Dempsey, 2008).

As evident from the foregoing discussion, a challenge exists in establishing a single etiology in fatigue as numerous factors are involved and the factors may be interdependent (Fitts, 1994; Lambert, St Clair Gibson, \& Noakes, 2005). Compartmentalization of specific causes has clouded understanding the mechanisms in and the site of fatigue (Brooks et al., 2005). To further complicate the matter, fatigue seems to vary with the nature of activity (task specificity), training and physiological status of an individual (aerobically trained versus untrained) and the environmental conditions (e.g., heat leads to quicker onset of fatigue), (Amann \& Calbet, 2008; Enoka \& Stuart, 1992).

Of all possible causes of fatigue, one of the common causes underlying both peripheral and central mechanisms is oxygen supply to the muscle and the brain. The following observations are relevant: (a) oxygen supply is needed to maintain an endurance related activity, (b) any delay in the supply of oxygen to meet the task demands results in metabolite depletion (PCr, ATP, glycogen etc) and metabolite accumulation (e.g., lactic acid build-up, accumulation of hydrogen and calcium ions), all of which are implicated in fatigue, (c) decrease in oxygen supply influences inhibitory feedback to the CNS, providing insight in to the perception of effort with progressive task performance, and (d) endurance training has been suggested to delay the 
onset of fatigue, under the assumption that increased endurance increases the task demands to be met earlier at the onset of exercise by increasing oxygen consumption.

In fact, oxygen supply plays an important role in predicting the onset of fatigue by way of two specific mechanisms, including (1) neuromuscular efficiency and (2) cardiovascular recovery. We now turn to a review of oxygen uptake kinetics and the role of kinetics in fatigue, information that will be central for the experimental hypotheses addressed in this study.

\subsection{OXYGEN KINETICS}

Oxygen uptake (VO2) is not proportional to the demands of oxygen at the onset of exercise but rather increases exponentially at exercise onset and continues to increase until a steady state in VO2 is reached. The dynamic behavior of oxygen uptake in the transition from rest to exercise

is termed oxygen kinetics (VO2 kinetics). Discussion of this phenomenon is pertinent to understanding the endurance or aerobic capacity of an individual as such capacity depends on (a) high capacity of the central circulation to deliver oxygen to the working muscles (transport), and (b) high capacity of the working muscles to use the oxygen available (peripheral extraction) (Figure 2-1). The effectiveness of each of these submechanisms determines availability of oxygen and use of aerobic metabolism to sustain a physical endurance activity. 




Figure 2-1. Oxygen Kinetics

\subsubsection{Central circulation}

The concept of central circulation is linked with the concept of transport. Transport of oxygen to working muscles is the prime function of central circulation. The main equation that can summarize the concept of oxygen consumption (oxygen uptake) is shown in Equation 2-1.

Oxygen uptake $\left(\mathrm{VO}_{2}\right)=$ Cardiac output $(\mathrm{Q}) *$ arterial-venous oxygen difference $\left(\mathrm{AVO}_{2}\right)$

Equation 2-1. Fick's equation summarizing oxygen consumption

Of concern for central circulation is "Q" in the equation. "Q" is cardiac output, defined here as the volume of blood ejected from the left ventricle of the heart per unit time (Brooks, et al., 2005; McCardle, Katch, \& Katch, 2001, 2007). Oxygen uptake is directly proportional to cardiac output. In other words, greater the cardiac output, the greater the oxygen uptake. In turn, two factors determine cardiac output (see Equation 2-2). 
Cardiac output $(\mathrm{Q})=$ Heart rate $(\mathrm{HR}) *$ Stroke volume $(\mathrm{SV})$

\section{Equation 2-2. Equation summarizing cardiac output}

Stroke volume is defined as the volume of blood pumped from the left ventricle per heart beat. As exercise intensity increases from rest, there is an increase in stroke volume until exercise intensity reaches about $50 \%$ of $\mathrm{VO}_{2 \max }$. At that point, stroke volume reaches a plateau. Mechanisms are as follows. Early in exercise, with increase in exercise intensity, venous return increases, which in turn increases the central venous pressure gradient. This increase results in concomitant increases in the diastolic volume of the left ventricle. Myocardial activity is thus increased, resulting in increased force of contraction of the left ventricle. In sum, greater venous return will result in greater volume of blood in the left ventricle, which will in turn increase the left ventricle's contractile force. Thus, more blood is pumped out per stroke (Brooks, et al., 2005; Myers \& Madhavan, 2001).

A second factor in central circulation is heart rate (HR). Heart rate is a readily available measure of central circulator response during aerobic exercise. Heart rate increases linearly with exercise intensity, but only levels off at $\mathrm{VO}_{2 \max }$. With increase in exercise intensity, there is an increase in oxygen uptake resulting in an increase in central circulatory response, which to a certain extent is influenced by the linear increase in HR. Subsequent to oxygen transportation, oxygen extraction by the working muscle follows.

\subsubsection{Peripheral extraction of oxygen (Use)}

A principal measure of the peripheral link in the oxygen kinetic chain is arterial-venous oxygen difference - $\mathrm{A}-\mathrm{VO}_{2}$ (see Figure 2-1 \& Equation 2-1). A- $\mathrm{VO}_{2}$ is defined as the difference in 
oxygen content in arterial versus venous blood. For every $100 \mathrm{ml}$ of blood passed through body or muscle tissue, $5 \mathrm{ml}$ of oxygen is extracted and used at rest. With increase in exercise intensity, the difference between arterial and venous blood increases resulting in an increase in $\mathrm{A}-\mathrm{VO}_{2}$. The major component parts of the peripheral link include regional oxygen delivery and muscle oxygen extraction (Myers \& Madhavan, 2001).

\subsubsection{Regional oxygen delivery}

Critical in the oxygen kinetics chain is circulation of blood to exercising muscles. The essential mechanisms in oxygen delivery are blood flow (cardiac output), blood pressure, and peripheral resistance (defined as resistance of peripheral vasculature in the systemic circulation) (Equation 2-3).

Blood pressure $=$ cardiac output $*$ peripheral resistance

Equation 2-3. Equation demonstrating law of hemodynamics

During physical activity, peripheral resistance decreases due to increased blood flow to the working muscles and the vasodilation of the working muscles. Blood pressure increases with increase in physical activity to maintain blood flow to heart and brain in addition to maintaining blood flow to working muscles (Brooks, et al., 2005; Myers \& Madhavan, 2001). Blood pressure increases due to increase in cardiac output and outweighs the decrease in peripheral resistance (Brooks et al., 2005). With oxygen delivery to the working muscles established, the next sequential step in the oxygen kinetics chain is the extraction of oxygen by the working muscle/ tissue. 


\subsubsection{Muscle oxygen extraction}

Muscle oxygen extraction is dependent on the diffusion gradient from the capillary to the muscle fiber (see Figure 2-2) (Myers \& Madhavan, 2001). With increase in exercise intensity, there is an increase in aerobic metabolic demand resulting in a widening of the diffusion gradient and thereby an increase in flow from capillary to muscle fiber. In addition to the diffusion gradient, another mechanism that increases oxygen extraction is known as the "Bohr effect". This effect, which involves loading or unloading of oxygen by hemoglobin $(\mathrm{Hb})$, is influenced by three factors: acidosis, body temperature and partial pressure of carbon dioxide $\left(\mathrm{PCO}_{2}\right)$. Increase in exercise intensity results in an increase of all three factors. With increase in acidosis and temperature, hemoglobin $(\mathrm{Hb})$ in blood loses affinity for oxygen. As a result, the number of oxygen molecules transferring to the fiber from the capillaries increases. In addition, with an increase in acidosis, oxygen saturation drops in venous blood. However, increased acidosis increases arterial blood saturation, resulting in significant extraction of oxygen from arterial blood. Thus, mechanisms including increased diffusion and the Bohr effect collectively widen the arterial-venous oxygen difference with exercise, thus increasing the extraction of oxygen by the working muscle.

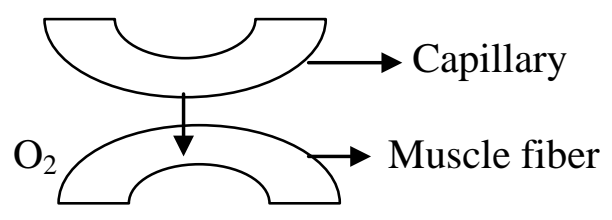

Figure 2-2. Diffusion of oxygen from capillary to muscle fiber 


\subsubsection{Take-home point regarding the oxygen kinetic chain}

The crucial links to understand oxygen uptake kinetics for a given activity are central circulation and peripheral extraction. During exercise and with increasing exercise intensity, oxygen transport to the muscle and oxygen extraction by the muscle determines the oxygen uptake required for that particular task. The amount of blood pumped from the left ventricle and the amount of oxygen extracted from the blood by the muscle fiber determine the efficiency of oxygen uptake for a particular task. For example, if the central circulator pumps adequate blood supply, but the working muscles are not capable of extracting oxygen for the particular activity, there is a dysfunction at the peripheral level, thereby slowing the $\mathrm{VO}_{2}$ kinetics. Poor oxygen extraction at the level of the muscle can be due to (a) decreased blood flow through the capillaries due to increased constriction of working muscles, (b) increased neuromotor recruitment of more muscles at the same time or differing times for a given task, (c) possible cocontraction of agonist and antagonist muscle groups, or (d) muscle membrane repair or damage (Hortibagyi, Finch, Solnik, Rider, \& De Vita, 2011). All of the mechanisms involved in poor oxygen extraction at the muscular level pertain to neuromuscular efficiency. Hence neuromuscular efficiency is the broad term that will be used throughout the document to indicate poor oxygen extraction by working muscles. With poor efficiency, metabolic cost (energy expenditure [oxygen consumed] standardized to the rate of the task) for a task is increased. On the other hand, if there is a dysfunction within the left ventricle, there is a decrease in the amount of blood pumped and hence there is not enough blood delivered to the muscle fibers from which oxygen can be extracted. Hence, a problem arises with central circulation thereby slowing $\mathrm{VO}_{2}$ recovery kinetics and affecting cardiovascular recovery issues (Brooks, et al., 2005). In that case, recovery time following a task increases. Of interest for exercise physiology in general and the 
present setting in particular, therapeutic implications differ for fatigue induced by poor efficiency during a task versus fatigue induced by poor recovery following a task (Poole, Barstow, McDonough, \& Jones, 2008). In the former case, an individual might receive training in task efficiency. In the latter case, an individual might be encouraged to undergo an overall cardiovascular (aerobic) training that is not necessarily task specific.

Two other concepts that provide insight into oxygen uptake kinetics are oxygen deficit and excess post exercise oxygen consumption (EPOC) and their relation to fatigue. These concepts are discussed next.

\subsubsection{Oxygen Deficit}

$\mathrm{VO}_{2}$ kinetics is defined as the behavior of $\mathrm{VO}_{2}$ uptake in the transition from rest to exercise. Prior to oxygen uptake, to meet the expenditure demands for any physical activity, the immediate energy source derives from the anaerobic breakdown of ATP, specifically the high-energy phosphates, resulting in a lag in oxygen consumption early in exercise. With continued exercise, hydrogen becomes available for oxidation and thereby oxygen consumption is proportional to exercise intensity until a steady state is achieved. Steady state is a balance created between energy required by the working muscles and the amount of ATP produced and available from aerobic metabolism (McCardle, et al., 2007). Stated differently, steady state oxygen consumption is identified as the exercise intensity at which oxygen supply is equal to oxygen demand. During steady state, the physical activity is achieved through aerobic metabolism. However, the period prior to achievement of steady state, when there is a lag in oxygen consumption is termed "oxygen deficit" (see Figure 2-3). Specifically, oxygen deficit is defined as the difference between the total oxygen consumed during exercise and the total that would have been consumed 
had steady state aerobic metabolism been achieved at the onset of exercise (McCardle, et al., 2007). The amount of oxygen deficit incurred at the beginning of exercise depends on cardiovascular conditioning. Oxygen deficit incurred at the onset of exercise is greater in untrained individuals than aerobically trained athletes, due to the rapid achievement of steady state in aerobically trained athletes (Jones \& Carter, 2000). In these individuals, aerobic metabolism is greater in the early stages of exercise due to a rapid increase in overall blood flow (cardiac output) and a greater regional delivery of blood to the working muscles (McCardle, et al., 2007). Further, the amount of oxygen deficit incurred at the beginning of each exercise is also dependent on the intensity of the exercise performed. Moderate to intense aerobic exercise requires a longer time to achieve steady state and hence creates a greater oxygen deficit than less intense exercise.

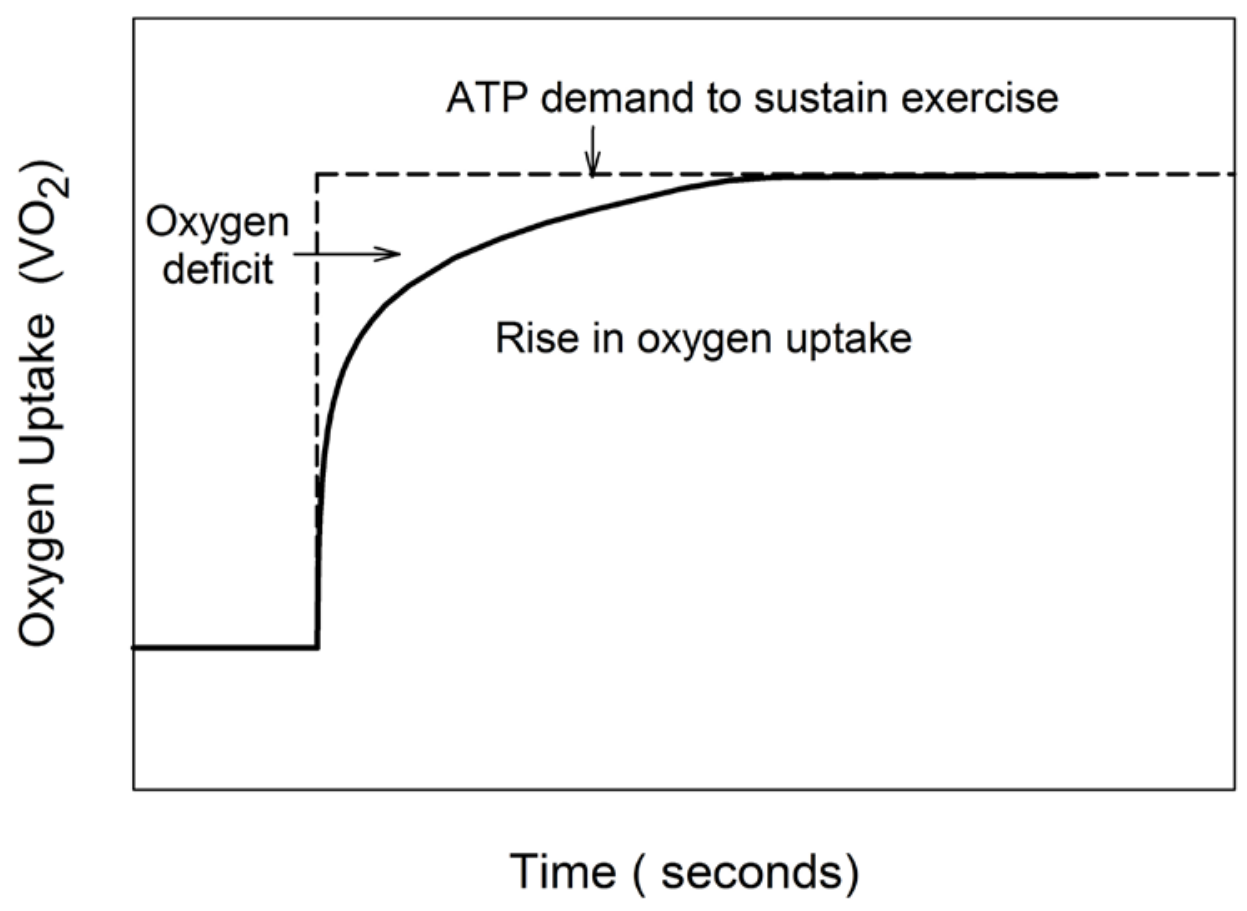

Figure 2-3. Oxygen uptake response at the onset of exercise 


\subsubsection{Oxygen deficit and its relation to fatigue}

High-energy phosphate sources (PCr) and anaerobic glycolysis, which involves non-oxidative mechanisms, are essential to generate energy for continuation of exercise until the rate of oxidative mechanism meets the required level. However, there are some negative consequences in the utilization of these mechanisms. Greater oxygen deficit is correlated with increased anaerobic glycolysis, resulting in reduced concentrations of PCr and possibly ATP, and increased concentrations of metabolic by-products such as ADP, inorganic phosphate, lactate and hydrogen ions in the contracting muscles (Allen, et al., 2008; Fitts, 1994; Myburgh, 2004; Sahlin, 1992). Since anaerobic glycolysis is a relatively inefficient process, muscle glycogen stores will be more rapidly depleted than if the same amount of ATP were generated oxidatively. Most of these mechanisms are associated and implicated with fatigue defined as declining ability to sustain an activity. Such mechanism may point to neuromuscular inefficiency of the working muscles. Additionally, larger oxygen deficit at the onset of exercise will result in a longer recovery time from the activity, as discussed next.

\subsubsection{Excess post exercise oxygen consumption (EPOC) and relation to fatigue}

Following physical activity, the physiological changes induced by activity do not immediately return to resting/baseline values. The time required for such changes to return to preexercise or baseline levels is defined as the recovery period. In order for physiological changes to recover, energy is required. Only in the presence of ATP is recovery possible and hence the energy required for recovery is supplied by aerobic metabolism. Stated differently, oxygen is consumed even after exercise ceases in order to return the physiological changes to resting levels. Oxygen consumption in recovery is always greater than the resting levels and is an indicator of "oxygen 
debt” (Hill, Long, \& Lupton, 1924). Of note, this term “oxygen debt” has been the subject of debate and is now usually replaced by the term "excess postexercise oxygen consumption" (EPOC) (Bahr \& Sejersted, 1991; Gaesser \& Brooks, 1984; McCardle, et al., 2001, 2007). The magnitude of EPOC is approximately equal to the amount of oxygen deficit incurred at the onset of exercise (McCardle, et al., 2001, 2007). Greater EPOC points to underlying cardiovascular recovery or cardiovascular fitness issues. In recovery, there are two important components - fast and slow components - that depend on the intensity of exercise. With mild aerobic exercise, depending on the aerobic conditioning of the individual, steady state is reached faster with very limited reliance on the anaerobic pathway for energy. Hence recovery from mild aerobic/ less intense exercise consists of only one component, namely the fast component of recovery. In contrast, recovery from intense, strenuous exercise follows a different recovery pattern. In addition to the fast recovery component, there is also a slow component (Poole, Barstow, Gaesser, Willis, \& Whipp, 1994). The reason is that with intense exercise, greater oxygen deficit is incurred due to increased reliance on anaerobic metabolism. Metabolic changes are increased including substrate depletion and increased concentrations of metabolic by-products. Hence, recovery takes longer (McCardle, et al., 2001, 2007). Aerobic training improves recovery rate by decreasing oxygen consumption during recovery (Tomlin \& Wenger, 2001).

Aerobic training increases the ability of the heart to pump more blood per unit time, and accelerates recovery. Poor cardiac output by the heart is an indication of poor cardiovascular fitness resulting in delayed recovery, which is a characteristic of sustained fatigue in individuals following an intense physical activity. Furthermore, aerobic training also enhances recovery by improving lactate removal and replenishment of phosphocreatine (Tomlin \& Wenger, 2001). To summarize, oxygen uptake $\left(\mathrm{VO}_{2}\right)$ plays a significant role during exercise and following exercise 
during recovery. The next section will address a sensitive measure to study oxygen uptake during and after exercise.

\subsection{MEASUREMENT OF MUSCLE FATIGUE}

Muscle fatigue has been assessed in different ways (Bigland-Ritchie, 1981; Bigland-Ritchie, Jones, Hosking, \& Edwards, 1978; Kent-Braun, 1997). Different measurement techniques are employed to identify different sites of failure in the muscle contraction process (Kent-Braun, 1997). One complication is that assessment of muscle fatigue has also been based on different definitions of fatigue (Vollestad, 1997). Some measurement techniques (e.g., electromyography, near infrared spectroscopy, etc) result in compartmentalization of mechanisms underlying fatigue, clouding the interpretation about potential sites of fatigue. In contrast, gas exchange analysis is a more promising option in some cases. This analysis provides a global, continuous measure by obtaining oxygen consumption during and after an activity, providing valuable information to delineate underlying essential mechanisms of fatigue: neuromuscular efficiency seen by metabolic cost for the task and cardiovascular recovery issues - seen by metabolic recovery time. The next section provides more information about this assessment procedure.

\subsubsection{Gas exchange analysis}

Gas exchange analysis has been used specifically to determine the metabolic cost associated with a physical task, providing information about both neuromuscular efficiency for a given physical activity and task recovery, providing insight in to cardiovascular recovery. Gas exchange, both the volume of oxygen consumed and carbon dioxide produced during and after exercise is 
measured using open circuit calorimetry. This calorimetry is an indirect form, the rationale being that all energy-releasing reactions in the body ultimately depend on oxygen use. Hence, measures of oxygen consumption (VO2) provides an indirect yet accurate measurement of metabolic cost, defined as the amount of oxygen consumed standardized to task rate.

As noted, information provided by gas exchange analysis is two-fold: (a) information is provided about the metabolic cost for a given task which is dependent on oxygen extraction by the working muscles and thus their efficiency and (b) information is provided about the recovery process following task completion which is dependent on cardiac output and circulatory response. A direct relationship exists between levels of oxygen uptake and onset of fatigue, where onset of fatigue is defined as increase in levels of oxygen uptake during an exercise (Poole, et al., 1991; Ratkevicius, Stasiulis, Dubininkaite, \& Skurvydas, 2006; Reybrouck, Vangesselen, Mertens, \& Gewillig, 2007). If oxygen uptake is slower during a given physical activity, producing a delay/lag to achieve steady state, the onset of fatigue is earlier, resulting in a decrement in muscular force output (Ratkevicius, et al., 2006; Reybrouck, et al., 2007).

Metabolic cost is determined by energy expenditure, the amount of oxygen consumed standardized by task rate for a given task. This ratio provides a measure that can be compared across people irrespective of the rate of task performance. Greater metabolic cost relates to poor neuromuscular efficiency. Data to this effect were provided by Ratkevicius and colleagues (2006), who measured oxygen cost during ergometer bicycling under two set-up conditions: (a) fatiguing condition and (b) control condition. Drop jump exercise prior to cycling was used to induce muscle fatigue in the fatiguing condition. Following the fatiguing set-up, oxygen uptake was increased during the biking protocol, indicating that oxygen cost increased with fatigue for the particular physical activity (Ratkevicius, et al., 2006). 
In a study on children with complaints of exertional fatigue, body-weight corrected oxygen uptake $\left(\mathrm{VO}_{2}\right)$ was measured during an incremental submaximal exercise. Oxygen uptake was significantly higher in children with complaints of exertional fatigue compared to age matched healthy controls. The authors suggested that an increase in oxygen uptake during physical activity indicates poor neuromuscular efficiency during task performance and thereby results in premature fatigue (Reybrouck, et al., 2007). In other words, individuals with complaints of fatigue demonstrate inefficient oxygen uptake during exercise, suggesting a direct relationship between oxygen uptake and fatigue. Similar results showing increased oxygen uptake during fatiguing exercise at high intensity were obtained (see also Poole, et al., 1991).

To summarize, gas exchange analysis, specifically, oxygen consumption during and after a task provides an indirect measure of aerobic metabolism. With this analysis, the extraction of oxygen by the muscles (peripheral extraction link) during the task gives us an indication of energy expenditure during the task, which provides insight into neuromuscular efficiency. Recovery from the task will rely on the heart's ability to pump blood to muscles (central circulator link) used during the task, and recovery characteristics can thus provide insight into a subject's cardiovascular fitness. Additionally, exploring oxygen uptake kinetics provides an insight on pattern of energy use over time for the task and their ability to manage the task. These points are central for the proposed research study.

\section{$2.5 \quad$ TREATMENT OF FATIGUE}

Treatment strategies in muscle fatigue can be classified under two broad categories. One focus has been pharmacological, including medications to (a) boost certain kind of nutrients, and (b) 
influence neurotransmitters in the brain. The pharmacological approach is based on proposed mechanisms underlying fatigue, centered on the depletion of metabolic substrates. Another treatment strategy involves an indirect method to deal with muscle fatigue. This method is linked to physical training with specific attempts to enhance task efficiency, task endurance (delay of fatigue onset), or both - as both approaches have been implicated in alleviating fatigue. Background information is as follows.

\subsubsection{Efficiency training}

Efficiency relates to the amount of energy required to perform a particular task to the actual work accomplished. Performance efficiency can be regulated by physiological or mechanical factors. Physiological factors including mitochondrial capacity for oxygen extraction are enhanced with aerobic training. On the other hand, efficiency can also be related to mechanical factors including technique (specificity and skill acquisition) and choice of equipment. Technique, including specificity in terms of appropriate muscle selection and muscle coordination, are as important for performance as the physiological capacity of an individual (Brooks, et al., 2005). Specific muscle groups need to be trained in order to prevent participation of muscle groups not required for a particular physical activity allowing for efficient performance. For example, efficient cyclists pedal smoothly at high revolutions per minute without engaging muscle groups that do not contribute to pedaling speed. Swimmers are required to use strokes that provide efficient propulsive force and minimize drag. Therefore, it is important for athletes to choose the right technique for efficient performance, minimizing fatigue.

Another strategy that plays a role in enhancing efficiency in performance is pacing. The right pace will enable an individual to perform longer for at least two reasons. First, pacing will 
influence how one perceives the effort of an exercise. Second, pacing will influence muscle glycogen supply. Choice of appropriate pace will facilitate "pay as you go" mechanism with aerobic means with less reliance on anaerobic means (muscle glycogen supply) (St Clair Gibson, et al., 2006).

Recovery between exercises is another important strategy for efficient performance. The length of recovery interval between exercise or physical bouts affects recovery. If insufficient time is provided for recovery between exercise bouts, ATP/PCr stores may not be fully restored and accumulated lactate may not be removed. If exercise continues in this instance without full recovery, efficiency in subsequent physical activity is impaired in turn affecting performance (Tomlin \& Wenger, 2001; Wooton \& Williams, 1983). Hence it is recommended that adequate recovery interval is provided between exercise bouts for enhanced performance and minimized fatigue. Aerobic training also influences recovery from exercise and the next section provides more information on endurance training in delaying the onset of fatigue.

\subsubsection{Endurance training}

Aerobic training is one strategy that has long been used in the exercise physiology domain to increase endurance, and enhance performance, and is an indirect approach to decrease or delay the onset of fatigue.

Aerobic training increases oxygen delivery by increasing muscle blood flow, and is achieved by elevated cardiac output (Ekblom \& Hermansen, 1968), increased capillarization of muscle tissue (Anderson \& Hendriksson, 1977; Saltin \& Rowell, 1980), and improved vasodilation (Sinoway, Musch, Minotti, \& Zelis, 1986). Blood volume and total hemoglobin volume also increases with endurance training (Brooks, et al., 2005). Further, accumulation of 
metabolites such as lactic acid and $\mathrm{H}^{+}$ions can be attenuated and replenishment of glucose and resynthesis of phosphocreatine can be augmented with increase in oxygen supply, decrease the reliance on anaerobic glycolysis during exercise (Brooks \& Donovan, 1983; Colliander, Dudley, \& Tesch, 1988; Holloszy \& Coyle, 1984). In sum, endurance training enhances oxygen delivery to the working muscle and the brain, thereby decreasing early onset of fatigue due to hypoxia.

A significant impact of endurance training is observed during recovery from exercise. During recovery, depleted resources need to be replenished, accumulated metabolites are removed and body's metabolism in general is returned to the pre-exercise state. In addition to adequate oxygen supply during physical activity, oxygen is also required following the completion of exercise. As noted, oxygen uptake at rest following exercise is elevated and is termed excess post exercise oxygen consumption (EPOC) (Bahr \& Sejersted, 1991; Gaesser \& Brooks, 1984). The excess oxygen is utilized in the replenishment of glucose, and phosphocreatine in the muscles for future use. In addition to the removal of accumulated lactate and $\mathrm{H}^{+}$ions during recovery, other body related changes during activity, including the cost of increased cardiac and respiratory functions, and elevated body temperature need to be restored for metabolism to return to pre-exercise levels (Brooks, Hittelman, Faulkner, \& Beyer, 1971; Gaesser \& Brooks, 1984; Sahlin, 1992). The process of recovery is enhanced and the time to return to pre-exercise state is shortened with endurance/aerobic training (Tomlin \& Wenger, 2001).

Endurance training results in cardiovascular adaptations that enhance the central link in the oxygen kinetic chain, which is key in transportation and supply of adequate oxygen to meet the physical demands of an activity resulting in cardiovascular adaptations. Specifically, the left 
ventricle's size is increased to hold more blood volume and the capacity to pump more blood with every beat is enhanced (Brooks, et al., 2005; McCardle, et al., 2001, 2007).

\subsection{SUMMARY}

Fatigue in the exercise physiology domain has been investigated at various levels. Different definitions prevail for fatigue based on the different schools of thought, including schools emphasizing peripheral fatigue, central fatigue, or a combination of both. Similarly, different mechanisms underlying fatigue have been proposed and various methodologies have been used to assess its mechanisms depending on the school of thought. Although various mechanisms are proposed, the focus here emphasizes two specific metabolic mechanisms including (a) poor neuromuscular efficiency of the working muscle, (b) poor cardiovascular recovery from exercise. A third option would involve (c) a combination of these two mechanisms. Within this framework, rational treatments to delay the onset of fatigue, thereby increasing endurance, have

focused on (a) increasing the efficiency of the working muscles or by (b) increasing cardiovascular efficiency to increase the circulation response to these muscles. This framework lays the groundwork for the present investigations on mechanisms that may underlie vocal fatigue. Integration of findings from exercise physiology and voice is the topic of the next chapter. 


\subsection{INTERGRATING EXERCISE PHYSIOLOGY PRINCIPLES WITH THE VOICE DOMAIN}

In this chapter, the focus is integration of principles on fatigue garnered from the exercise physiology domain (Chapter 2) with information from clinical voice science. As noted in the Introduction to this document, to date mechanisms underlying vocal fatigue have evaded specification. It seems reasonable to explore underlying mechanisms extrapolating principles from the exercise physiology domain. The specific framework pertains to the relevance of neuromuscular inefficiency and poor cardiovascular recovery in physical fatigue. This framework is novel as pertains to clinical voice, and may lay the foundation for understanding vocal fatigue and its rational treatment.

To reiterate, the predominant mechanisms underlying fatigue in relation to metabolic mechanisms related to physical performance include (a) poor neuromuscular efficiency, as a result of poor extraction of oxygen by the working muscles and (b) poor cardiovascular recovery, as a result of poor circulatory response. In the exercise physiology domain, gas exchange analysis has been employed predominantly to investigate these mechanisms in fatigue (Hogan, Kohin, Stary, \& Hepple, 1999; Kemps et al.,Poole, et al., 1991; Reybrouck, 2007; Ratkevicius, et al., 2006). This type of analysis involves continuous sampling and ongoing measures of energy expenditure during task performance and cardiovascular recovery from the task, as reflections of fatigue (Reybrouck, et al., 2007; Ratkevicius, 2006). To date, this approach has not been utilized 
to exploring vocal fatigue. Especially in light of the paucity of physiological evidence surrounding vocal fatigue this approach would seem to be a good starting point for a fresh approach to understanding mechanisms in vocal fatigue. Specifically, gas exchange analysis should help elucidate metabolic mechanisms underlying fatigue pertaining to (a) neuromuscular inefficiency (i.e., oxygen extraction by working muscles) and (b) poor cardiovascular recovery (i.e., oxygen transport and delivery).

Moreover, if fruitful, this approach should point to possible treatment options for vocal fatigue. Elucidating such underlying mechanisms influences the choice of differential treatment options to alleviate or delay the onset of fatigue. Two differential treatment approaches have been proposed and investigated in the exercise physiology domain: (a) improving efficiency by enhancing skill acquisition and appropriate recruitment of the working muscles and (b) improving circulatory response by increasing cardiovascular fitness and endurance.

Here, efficiency is defined as the ratio of mechanical work output to total metabolic cost, calculated by means of oxygen consumption (Brooks, et al., 2005). In efficiency training, the goal is to engage the muscles involved in physical activity to use the least amount of energy to accomplish a given amount of work. Efficient performance can be accomplished by increasing coordination among working muscles, changing performance pace, providing different mechanical aids (e.g., toe clips and clothing that enhance aerodynamics in cycling), (Brooks, et al., 2005). In the voice domain, vocal efficiency (dB SPL/cm $\mathrm{H} 2 \mathrm{O} \times \mathrm{l} / \mathrm{sec}$ )- defined as a quantitative measure of the ability of the larynx to convert subglottal pressure to acoustic power (Jiang, Stern, Chen, \& Solomon, 2004) - has been investigated in relation to the working muscles. For example, efficiency is known to vary with adjustments in glottal width (Slavit and McCaffrey, 1991, Titze, 1992), contraction of cricothyroid muscle (Koike \& Hirano, 1973, Titze, 
1992), and contraction of vocalis muscle (Hirano, 1974, Berke et al., 1990). Efficiency is also influenced by other factors including intensity and subglottal pressure (Schutte, 1980). Increased subglottal pressure requires recruitment of more muscles and decreases the glottal width between the vocal folds creating an inefficient neuromuscular pattern.

Thus, data are available pointing to muscular adjustments that affect vocal efficiency and thus potentially reduce vocal fatigue during task performance. What are lacking are data on the possible influence of cardiovascular fitness on vocal fatigue. In fact, the present study is the first that we are aware to even consider this rather unintuitive factor that may operate in vocal fatigue. As noted, cardiovascular fitness, which is a function of endurance training, increases cardiac output by increasing stroke volume, thereby increasing oxygen supply to the working muscle, in turn increasing the endurance to perform an activity (Brooks, et al., 2005). Endurance training increases circulatory response during exercise to enhance the quick supply of oxygen to meet the demands of the task. Additionally, endurance training also increases the circulatory response post exercise to aid in quick recovery from the task by replenishing depleted resources and eliminating metabolic byproducts to return the body to pre-exercise baseline levels (Brooks, Hittelman, Faulkner, \& Beyer, 1971; Gaesser \& Brooks, 1984; Sahlin, 1992; Tomlin \& Wenger, 2001). Thus, a question is raised about the potential contribution of cardiovascular fitness in vocal fatigue. The importance and the novelty of this question cannot be overestimated. If indeed, the present study can relate vocal fatigue to metabolic mechanisms relating to cardiovascular fitness, it will be the first to point to the seemingly far-fetched idea that cardiovascular training may be useful in the treatment of individuals with vocal fatigue. 
In this pursuit, we propose a conceptual framework for vocal fatigue, integrating pertinent information from both exercise physiology and voice domains. In brief, vocal fatigue may be induced at one or more of several levels indicated in Figure 3-1.

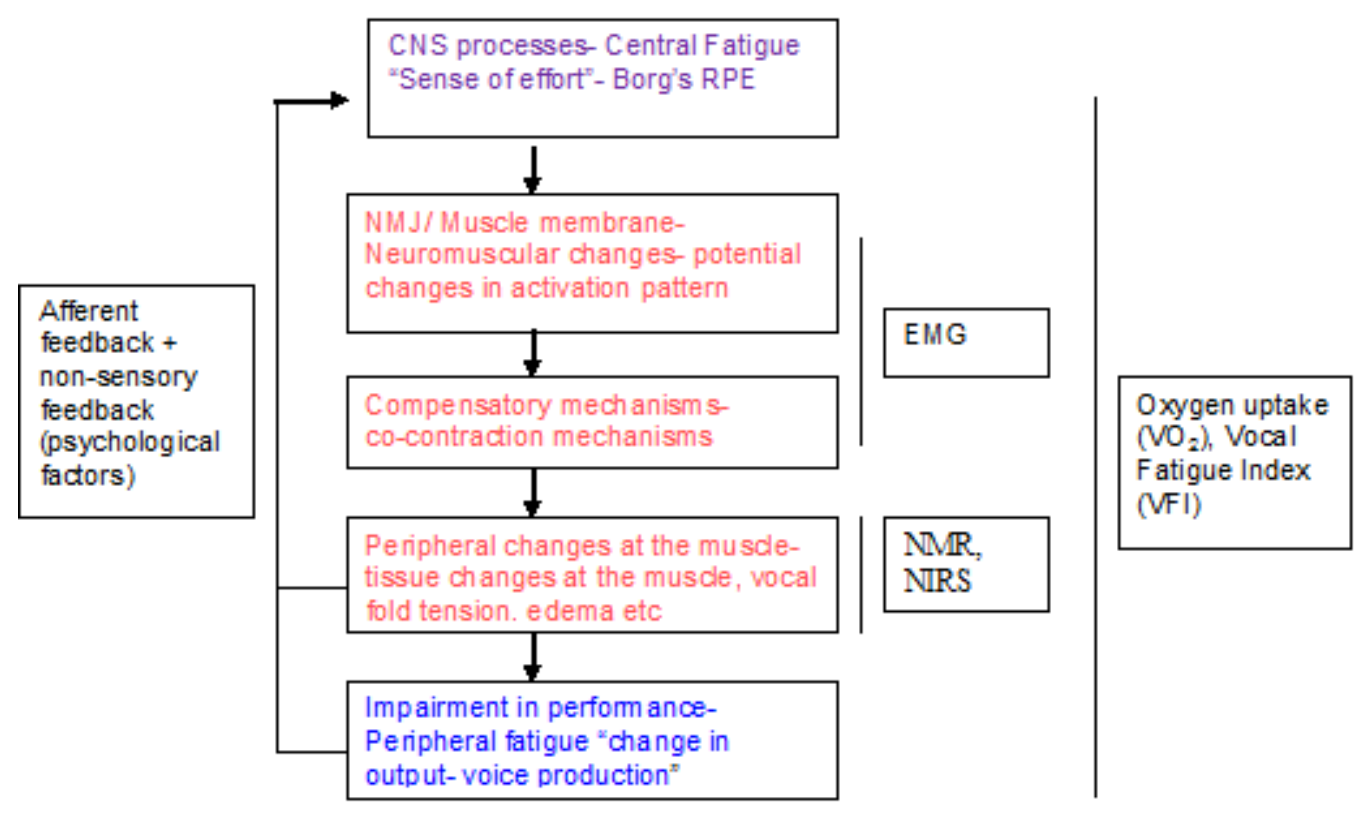

Figure 3-1. A conceptual Framework of Vocal Fatigue

Of particular interest for the present study are impairments seated in neuromuscular efficiency or cardiovascular recovery. The ultimate outcome of this framework is performance. There is impairment in performance with the onset of fatigue (indicated in blue- Figure 3-1). The onset of fatigue is influenced at various levels of the framework. For e.g., neuromuscular inefficiency (indicated in red- Figure 3-1) can be observed if there are additional muscles involved during the task or if there is a co-contraction of agonist and antagonist laryngeal muscles during task performance. Additionally, peripheral changes at the laryngeal tissue including vocal fold tension and edema also predict neuromuscular inefficiency. At the onset of 
fatigue from the peripheral system, afferent feedback is sent to the brain indicated by an increase in the effort (indicated in pink- Figure 3-1) to produce voice. Consequently, the brain initially provides additional recruitment of motor units to meet the demands of the task, but with increase in fatigue and inability to sustain the task, recruitment of motor units decreases to maintain homeostasis of body metabolism. This is seen as neuromuscular changes in the activation pattern. Each level in the framework can be isolated and studied by appropriate measures indicated on the framework. For example, oxygen consumption, as measured by gas exchange analysis, will be used as a global measure to provide insight into these potential mechanisms in vocal fatigue, including both inefficiency of the working muscles to extract oxygen (reflecting efficiency) and poor circulatory response (reflecting recovery). Other assessments that assess specific textboxes are as follows. Electromyography (EMG) can be employed to study specific muscle related changes (e.g., compensatory and co-contraction mechanisms, and potential changes in neuromotor activation pattern). Additionally, nuclear magnetic resonance spectroscopy (NMR) and near infrared spectroscopy (NIRS) will help identify specific tissue related changes (e.g., metabolite accumulation, oxygen saturation at the level of the working muscles). Perceived sense of effort sent as afferent feedback can be assessed using rating scales such as Borg's ratings of perceived exertion. What is not indicated in this framework is the physiological response of cardiovascular recovery, but can be measured using gas exchange analysis. However, as a first step in adapting principles from the exercise physiology domain, the present study will utilize gas exchange analysis to investigate the underlying mechanisms of vocal fatigue as proposed earlier, including neuromuscular inefficiency and cardiovascular recovery issues. Additionally, ratings of perceived exertion to identify sense of effort associated 
with vocal fatigue will be employed in the present study. The other measures including EMG and NIRS will be considered in future protocols of vocal fatigue.

A long-term goal is to adapt existing assessment and treatment paradigms used in the exercise physiology domain to the case of vocal fatigue. Specific aims for the present study are as follows.

\subsection{STATEMENT OF PURPOSE, SPECIFIC AIMS, EXPERIMENTAL QUESTIONS, AND HYPOTHESES}

It is an understatement to say existing literature on vocal fatigue reveals foundational gaps. Among them are (a) the lack of a precise and definite definition of vocal fatigue, (b) the lack of sensitive tools to identify individuals with vocal fatigue, (c) limited information about potential physiological mechanisms underlying vocal fatigue, and (d) lack of efficient and effective therapeutic strategies to prevent and treat vocal fatigue.

The present study is a first step in a long-term research program that aims to address these gaps. The ultimate goal is to identify effective and efficient treatment strategies for treating vocal fatigue in individuals who experience it. A preliminary step toward reaching that goal, addressed in the present study, is to explore metabolic mechanisms that may underlie vocal fatigue including neuromuscular inefficiency (metabolic cost for a task) and cardiovascular recovery issues (recovery time from the task).

In this study, individuals with possible vocal fatigue syndrome were identified using a

newly developed Vocal Fatigue Index (VFI; Nanjundeswaran, Jacobson, Gartner-Schmidt, Rosen, \& Verdolini Abbott, under review). The VFI is a paper-and-pencil questionnaire 
developed to identify individuals with and without vocal fatigue. Analyses based on VFI results for 200 subjects with voice disorders pointed to three factors that may underlie vocal fatigue: (1); Factor 1, relating to avoidance of voice use and tiredness of voice (11 items), (2) Factor 2, relating to physical discomfort with voice use (5 items), and (3) Factor 3, relating to improvement of voice symptoms or lack thereof with voice rest (3 items). Logistic regression analysis identified Factors 1 and 3, but not Factor 2, as predictors of presence or absence of chronic vocal fatigue. Higher scores on Factor 1 indicated increased vocal fatigue with voice use, whereas higher scores on Factor 3 indicated improvement of voice symptoms with voice rest. For purposes of the current study, scores from Factor 1 were used to identify subjects with likely vocal fatigue syndrome. Scores on factor 3 were not used since scores varied across subjects if symptoms improved or did not improve with voice rest.

Metabolic processes in participants with vocal fatigue, vocally healthy participants, and participants with superior cardiovascular training were assessed using gas exchange analysis. In that analysis, of specific interest were metabolic processes occurring during phonation, and metabolic processes occurring during a post-phonation recovery period. Specific Aims were:

\section{Specific Aim 1 (primary)}

(a) To identify gross metabolic cost (energy expenditure/rate of speech) during a vocal task and (b) to evaluate metabolic recovery times following task performance in individuals with vocal fatigue, in comparison to two control groups: (i) vocally healthy, vocally untrained, cardiovascular untrained individuals, and (ii) cardiovascular trained, vocally healthy individuals without voice training. The purpose of this aim was to determine if evidence could be 
shown regarding potential efficiency or recovery mechanisms in individuals with vocal fatigue. Hypotheses were:

- Metabolic costs during reading will be greater for subjects in the vocal fatigue group than for subjects in the two non-fatigue comparison groups implying a role of vocal inefficiency in vocal fatigue.

- Post-reading recovery times will be greater for subjects in the vocal fatigue group than for subjects in the two non-fatigue comparison groups, implying a potential role of poor cardiovascular fitness in vocal fatigue.

- Post-reading recovery times will be shorter for individuals in the cardiovascular trained group than for individuals in both vocal fatigue and vocally healthy groups, providing further evidence that cardiovascular fitness may be (inversely) relevant to vocal fatigue.

(Exploratory to S.A.1): To explore oxygen uptake kinetics and recovery kinetics (EPOC) in individuals with vocal fatigue in comparison to two non-fatigue groups. Hypotheses were:

- Oxygen uptake kinetics will be slower and prolonged during the task implying neuromuscular inefficiency and the recovery kinetics will be longer for the vocal fatigue group implying poor cardiovascular fitness compared to other non-fatigue groups.

Specific Aim 2 (secondary aim 1)

To determine perceived phonatory effort/perceived exertion for the vocal tasks and to determine if a correlation exists between perceived phonatory effort/perceived exertion using the Borg 
scale and gross metabolic cost for the vocal tasks. The purpose of this aim was to determine if an easily obtainable perceptual proxy measure can be used to estimate gross metabolic costs for phonation. The hypothesis was:

- A significant positive correlation exists between perceived phonatory effort/perceived exertion and gross metabolic cost for the vocal tasks.

Specific Aim 3 (secondary aim 2)

To determine heart rate as a measure of cardiovascular fitness and if such measure correlates with recovery time following the vocal task. The purpose of this aim was to obtain potential impressions about the role of cardiovascular training history in recovery from the vocal task. The hypothesis was:

- A significant positive correlation will be shown between estimates of cardiovascular fitness and metabolic recovery from a vocal task. Evidence to this effect would further speak to a potential role of cardiovascular fitness in recovery.

Specific Aim 4 (secondary)

To determine vocal efficiency of the three groups and if measures of vocal efficiency correlate with gross metabolic cost for the vocal task.. The purpose of this aim was to illuminate possible contribution of vocal/laryngeal inefficiency during performance of a vocal task. The hypothesis was:

- A significant negative correlation will be obtained between vocal efficiency and gross metabolic cost for a vocal task. Evidence to this effect would speak to the role of neuromuscular inefficiency or efficiency during the task. 
Specific Aim 5 (secondary)

To determine if correlations exist between Factor 1 of the VFI (avoidance of voice use and tiredness of voice) and metabolic cost during the vocal task and between Factor 3 of the VFI (improvement of voice symptoms or lack thereof with voice rest) and post-task recovery in the vocal fatigue group. The purpose of this aim was to determine if results for Factors 1 and 3 might serve as simple paper-and-pencil values- clinical tool to identify differential treatment options reflecting metabolic cost during a vocal task and during recovery from the task. Hypotheses were:

- A significant positive correlation will be shown between factor 1 on the VFI and gross metabolic cost for the task. A significant negative correlation will be shown between factor 3 on the VFI and recovery time following the task in individuals with vocal fatigue. Findings to this effect would indeed suggest that a simple screening tool, the VFI, might be used to assess risk factors for efficiency versus cardiovascular issues in individuals complaining of vocal fatigue. 


\subsection{RESEARCH DESIGN AND METHODOLOGY}

\subsection{PARTICIPANTS}

Three groups of female participants in the age range of 18-45 years were recruited for the study. Groups were: (1) Individuals with complaints of vocal fatigue with no particular cardiovascular conditioning (vocal fatigue group; VF), (2) vocally healthy, vocally untrained and cardiovascular unconditioned individuals (vocally healthy group; VH), and (3) cardiovascular trained based on self-report of good endurance training, vocally healthy individuals without voice training (cardiovascular trained group; CV). Henceforth, all groups will be referred using the acronyms indicated parenthetically. Only females were recruited for the study, as voice disorders in general are approximately three times more frequent in females than in males (Morton \& Watson, 1998; Rousseau, et al., 2004; Russell, Oates, \& Greenwood, 1998; Smith, Kirchner, Taylor, Hoffman, \& Lemke, 1998). Additionally, females experience more vocal fatigue symptoms compared to men (Russell et al., 1998; Smith, Gray, Dove, Kirchner, \& Heras, 1997; Smith et al., 1998).

Based on pilot data on seven $\mathrm{VH}$ individuals and three individuals with VF, with alpha set at .05 , and an effect size of $\mathrm{f}=.458$, a total sample size of 24 was necessary to achieve a desired power of $80 \%$. A total of 24 participants were thus recruited for the vocally healthy group and vocal fatigue group with 12 in each group. Additionally, after the initial proposal had been finalized, as an afterthought, 8 participants were recruited in the CV group. The reason for 
unequal number of participants across groups was primarily because CV group was added after the fact, and we simply to ran out of resources to recruit more participants. Additionally, analysis indicated that CV group displayed minimum variability in metabolic cost for the vocal task and metabolic recovery time suggesting the need to recruit less participants.

This, in total, across all three groups, 32 participants were recruited and enrolled in the study. Mean ages were 32.7 yrs ( $\mathrm{SD}=9.73,20-45 \mathrm{yrs}$ range) for the VF group, $26.3 \mathrm{yrs}(\mathrm{SD}=$ 5.64, 21-41 yrs range) for the VH group, and 22.9 yrs ( $\mathrm{SD}=3.27,19-28$ yrs range) for the CV group.

All participants were recruited using IRB-approved advertisements (See Appendices A and B). The advertisements were distributed in University settings (East Tennessee State University), school settings (Johnson City Public schools) and the community at large. Participants provided information about inclusionary and exclusionary criteria listed below by filling out an online survey created on google docs.

(https://docs.google.com/spreadsheet/viewform?formkey=dE44bXEyYjR0Z3RGX2JZNTZqM0J fMkE6MQ)

\subsubsection{Inclusion criteria}

For all groups, general inclusionary criteria included: (a) female between the ages of 18-45 years (the upper limit of 45 years was chosen to restrict recruitment to females in a relatively stable hormonal age range), (b) no known degenerative or other medical conditions that would affect voice, with the exception of seasonal allergies (and their treatment), asthma or laryngopharyngeal reflux and their treatments, (c) self-report of normal reading fluency in English, and (d) self-report of completion of high school education. 
Specific inclusionary criteria for the different groups were as follows: For the VF group, inclusionary criteria were: (a) a high score on Factor 1 in the VFI ( $\geq 24$; Nanjundeswaran et al., under review; see Appendix C), (b) no report of any enrollment in any voice therapy program currently or in the past, and (c) no obvious evidence of organic pathology of any origin affecting voice, based on rigid endoscopic examination by a board certified otolaryngologist. Criteria to consider the vocal folds free of organic pathology included no visible lesions, normal arytenoid motion on adduction and abduction and appropriate phonatory closure. Although not specifically inclusionary or exclusionary, of 12 participants in the VF group, 5 had some vocal training, including two vocal music teachers and three students majoring in vocal music. In terms of cardiovascular training, 6 participants in the VF group were sedentary. The remaining 6 participants had exercise and fitness histories similar to those in the $\mathrm{VH}$ group $(<20$-30min of aerobic activity $<$ than three days per week).

For VH and CV groups, inclusionary criteria were: (a) no current self-perceived voice problem, or any history of a voice problem lasting more than 2 weeks in the past, (b) low score on the Vocal Fatigue Index Factor 1 (VFI) $(\leq 5)$, and (c) healthy vocal folds including normal structure and function based on visual rigid endoscopy by a board-certified otolaryngologist using the same criteria as for the VF group. The difference between the $\mathrm{VH}$ and $\mathrm{CV}$ groups related to cardiovascular training history. Specifically, to qualify for the VH group, participants had to report they exercised $<20$-30min per day less than three days per week over the preceding six months. To be recruited into the CV group, the individual had to report exercising 40-60min daily for at least five days per week and should have done so consistently in the past six months (American College of Sports Medicine guidelines; in McCardle et al., 2007, Brooks et al., 2005). 
None of the participants in either the VH or CV groups had any prior voice training except for occasional singing in choirs.

\subsubsection{Exclusion criteria}

Information about exclusion criteria was obtained from participants' self-reports on the online survey and confirmed on the day of in-office screening, unless otherwise noted. Exclusion criteria for all subjects were as follows: (a) evidence of acute or chronic upper respiratory infection at the time of testing; (b) active symptomatic allergies, laryngopharyngeal reflux disease or asthma at the time of testing based on self-report; (c) history of any cardiac, pulmonary, or neurological problems, specifically history or symptoms of systemic diseases known to affect the nervous or endocrine systems; (d) the use of medications known to affect the nervous and endocrine systems, voice (e.g., drugs that have a drying effect, anti-inflammatory drugs), or oxygen uptake (e.g., anticholinergic drugs; muscle relaxants) in the week prior to the experimental session. For every medication listed on the online screening form, to determine its potential effects, the medication was searched on the internet and if required, additional confirmation was obtained from the team's clinical knowledge (SLP, PT and board certified otolaryngologist).

An additional exclusionary criterion for all groups was current tobacco use, including smokeless tobacco use, within the preceding three months, by self-report. Further, any organic pathology potentially affecting the structure and function of voice production was a specific basis for exclusion (e.g., lesions, paresis, paralysis, etc) based on a rigid videostroboscopic laryngeal examination by a board certified otolaryngologist. 


\subsection{EXPERIMENTAL DESIGN}

The primary focus of the present study, stated in Specific Aim 1, involved a 2x3 mixed-model design. The first dependent variable was gross metabolic cost of reading standardized to the rate of speech, defined as the ratio of energy expenditure/ rate of speech (oxygen consumption $[\mathrm{ml} / \mathrm{kg} / \mathrm{min}] /$ words per minute $[\mathrm{wpm}]-\mathrm{ml} / \mathrm{kg}-\mathrm{w})$. The within-subjects factor was experimental task (reading at two levels: conversational and loud) and the between-subjects factor was the group (VF, VH, and CV). The second dependent variable was metabolic recovery from the vocal task. This variable was assessed in two ways: (1) recovery duration (seconds- time take for oxygen consumption in $\mathrm{ml} / \mathrm{kg} / \mathrm{min}$ to reach baseline levels), and (2) the percentage of subjects who achieved 50\% recovery by 1 and 2 min post reading. For both recovery variables, again, the within-subjects factor was again the experimental task (recovery from conversational reading and recovery from loud reading), and the between-subjects factor was group (VF, VH, and CV). The independent variables were experimental phase and subject groups. Secondary variables that would be assessed as outlined in Specific Aims 2, 3 and 4 included perceived phonatory effort/perceived exertion (defined as the rating of one's perception of vocal effort or discomfort using Borg's RPE following the reading tasks), cardiovascular fitness (defined as quick return of heart rate 1 minute following 3 minutes of exercise as measured using the Bench Stepping Test; lower heart rate indicates good cardiovascular conditioning) and vocal efficiency (the larynx's ability to convert subglottal pressure to acoustic power measured as $\mathrm{dBSPL} / \mathrm{CmH}_{2} \mathrm{O} \mathrm{x} \mathrm{l} / \mathrm{sec}$ ). 


\subsection{EQUIPMENT AND TOOLS}

Screening Phase. Equipment used for the screening phase included Vocal Fatigue Index (VFI, Nanjundeswaran et al., in review) and rigid videostroboscopy (KayPENTAX,Montvale, NJ, USA)

Experimental Phase. For the experiment proper, a portable gas exchange analyzer, MedGraphics VO2000 system (Minneapolis, MN, USA), a Dell Laptop with the Lee Silverman Voice Treatment (LSVT) Companion software ${ }^{\circledR}$ and its appropriate company manufactured microphone was used. Other equipment included the Phonatory Aerodynamic System 6600 (PAS6600) (KayPENTAX, Montvale, NJ, USA). Additionally, Borg's scale (RPE) was used during the experimental phase.

\subsection{EQUIPMENT CALIBRATION}

The MedGraphics VO2000 system used an auto-calibration mode that calibrates the analyzers using room air and proprietary software. The calibration process was initiated automatically prior to initiating each participant's data collection. For the PAS6600, the air-pressure tube and pneumotach were calibrated daily, per manufacturer instructions. The system automatically calibrated the air-pressure tube from the system’s “Options” drop down menu. To calibrate the pneumotach, a 1.0L syringe was attached to the airflow head and on system prompt, the plunger was pushed in one continuous motion for 2-4seconds resulting in a calibration value. Proper calibration value resulted in a value of $1.0 \mathrm{~L}+/-1-2 \%$. If proper calibration values were not 
obtained, troubleshooting was performed and calibration procedure repeated until proper values were obtained.

\subsection{EXPERIMENTAL PROCEDURES}

The Institutional Review Board of the University of Pittsburgh and East Tennessee State University (ETSU) approved the protocol before the study commenced. As indicated earlier, all participants were recruited by way of IRB-approved advertisements.

\subsubsection{Screening}

Information about the number of individuals involved in every phase of the study, from initial response through study completion, is provided in Figure 4.1. All individuals who indicated interest in participation via telephone or e-mail, were directed to a secure online survey developed for the purpose of this study, on google docs for initial screening purposes (https://docs.google.com/spreadsheet/viewform?formkey=dE44bXEyYjR0Z3RGX2JZNTZqM0J fMkE6MQ). The survey posed questions about demographic information (e.g., age range, gender, contact information- e-mail or phone as preferred), information about reading fluency, education, information on any speech, language, or voice issues, gag reflex, current medications, cardiovascular exercise and vocal training, and also required response to all questions on the VFI. Once the participants completed the survey, based on their eligibility, they were contacted and scheduled for an in-office screening. At the in-office screening, all participants first provided informed consent to participate in the study. Next, selected questions from the online survey, including questions about medical history, voice history, cardiovascular activity (type and 
duration of activity), and medication history were revisited and responses were confirmed to determine further eligibility in the study. Next, a board certified otolaryngologist performed a laryngeal examination to determine the health of the vocal folds. For all groups, observation of any visible lesions, poor or abnormal arytenoid mobility or inadequate glottal closure during phonation determined ineligibility for further participation. All qualified participants after screening, were scheduled for the actual experimental protocol on a different day, but within a week from the in-office screening. 


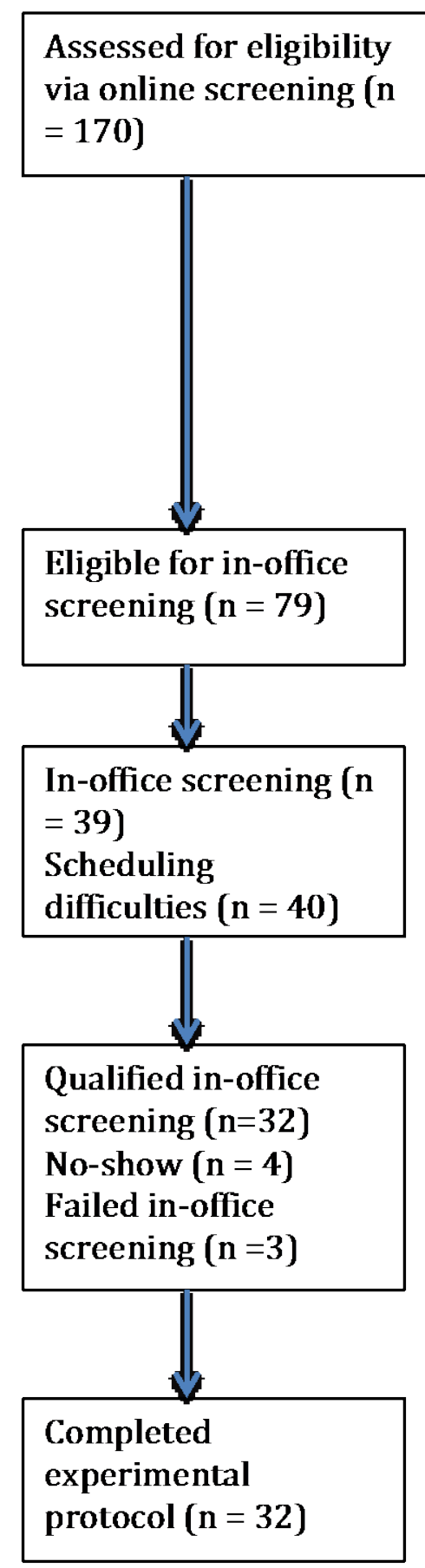

Excluded $(\mathrm{n}=91)$

VFI score $(n=42)$

Fitness $(n=26)$

Medication $(n=9)$

Age ( $\mathrm{n}=1)$

Smoking $(\mathrm{n}=5)$

Gag reflex $(n=1)$

Other $(n=7)$

Figure 4-1 Participant flow chart 


\subsubsection{Experimental set-up and training}

The entire experimental protocol was conducted on a day different from screening. Upon arrival, participants were seated comfortably on a desk chair facing a door. They were provided an outline of the experimental protocol for the day (see Table 4-1). They were also provided a brief overview of gas exchange analysis using the MedGraphics VO2000 system without revealing the intent of the study. Specifically, they were informed that a mask would be secured around their nose and mouth and a tube (preVent flow tube) would sit outside their mouth but would not interfere with breathing. Next, the device’s mask was secured around the participant's nose and mouth using a Velcro strap. The participant was asked to indicate whether the mask was comfortable, and adjustments were made until an affirmative answer was achieved while at the same time, placement was adequate for data collection purposes. Adequacy was confirmed by the presence of a good seal around the nose and mouth and by assessment of the feedback from gas exchange data captured every three to five seconds on expiration. Once the mask was secured, the microphone, a part of the LSVT Companion software ${ }^{\circledR}$, which was connected to a Dell Laptop to monitor loudness levels during reading was positioned at a distance of $30 \mathrm{~cm}$ and at a 45-degree angle from the corner of the mouth. The distance was measured using a tape provided with LSVT Companion software ${ }^{\circledR}$. Participants were asked to stay in that position to maintain the distance. Once that microphone set-up was established, participants were instructed to speak at their comfortable loudness for $30 \mathrm{sec}$ to 1 minute. The average intensity measured in $\mathrm{dB}$ sound pressure level was obtained and noted and would be used as the target for conversational reading tasks. Then participants were asked to increase their loudness by 10-15 $\mathrm{dB}$ sound pressure level over their conversational loudness level and produce their voice for 30 sec to 1 minute. If the intensity did not increase to meet the $10-15 \mathrm{~dB}$ sound pressure level 
change, participants were instructed to increase their loudness. Once the target intensity was achieved, the average intensity was noted and would be used as the target for the loud reading task. The next step was for participants to read at their target intensities for conversational and loud reading for 1-2 minutes for each condition until they felt comfortable producing their voice at those intensity levels. Participants were instructed to only speak during reading tasks and maintain target intensity levels during reading tasks during the experimental protocol. Additionally, participants were instructed that they would be primed via auditory feedback to increase or decrease their intensity during reading, if their intensity levels became softer or louder. Participants were then instructed that both vocal efficiency task and Bench Stepping test would be completed following the completion of gas exchange analysis. The entire protocol from training to completion lasted about an hour for all participants.

Table 4-1 Task order of the experimental protocol for the day

\begin{tabular}{|l|r|r|}
\hline $\begin{array}{c}\text { Gas Exchange Analysis } \\
\text { (Step 1) }\end{array}$ & $\begin{array}{c}\text { Vocal Efficiency Test } \\
\text { (Step 2) }\end{array}$ & $\begin{array}{c}\text { Bench Stepping test } \\
\text { (Step 3) }\end{array}$ \\
\hline
\end{tabular}

\subsubsection{Experimental Procedures}

For experimental procedures proper, a respiratory baseline was obtained. To obtain that baseline, participants were instructed to breathe in through their nose and out through their mouth. Baseline oxygen consumption was monitored during this process until steady-state oxygen consumption level was achieved for approximately one minute (i.e., until variation in oxygen consumption was $<2.0 \mathrm{ml} / \mathrm{kg} / \mathrm{min}$ ) using the MedGraphics VO2000 system. The time to 
achieve steady state varied anywhere between 90 seconds to 3 minutes. Following the achievement of steady state, the participants were asked to initiate their first reading task, conversational versus loud. Order of loudness conditions was counterbalanced across subjects. Participants read junior high school level passages (Sims, 1987a, 1987b; see Appendix D). As soon as the participant started reading, the LSVT Companion Software ${ }^{\circledR}$ was turned on to monitor and record speech intensity throughout the reading task. During the reading task, the experimenter used auditory feedback to coach/prime participants to either increase or decrease their loudness as necessary (e.g., primed "louder"- when volume dropped below the target loudness or "softer"- when volume increased above the target loudness). The first reading task was continued for five minutes to allow sufficient time for subjects to achieve steady state in oxygen consumption (ml/kg/min) (Mc Cardle et al., 2005; Russell, Cerny, \& Stathopoulos, 1998). The point of termination in the reading passage was noted to allow for post-hoc assessment of speech rate (words per minute - wpm). Speech rate was later used to obtain rate of oxygen consumed (energy expenditure/wpm) to determine metabolic cost. Following the first reading task, participants were given time to rest and recover. During this time, they were instructed to breathe in through the nose and out through the mouth until a 30-sec recovery plateau was achieved in oxygen consumption. Recovery was defined as the time taken for oxygen consumed to reach baseline value of oxygen consumption (within $.2 \mathrm{ml} / \mathrm{kg} / \mathrm{min}$ ). Typically, for this study, it was $<4$ min, but varied across subject groups. The second speech task did not commence until recovery to baseline was achieved, even if longer than the typical 4 minute duration. Once recovery was established, the same protocol was administered for the second reading task (conversational or loud, whichever level the participant had not yet used) and recovery from it. See Table 4.2 for experimental procedures. 
Table 4-2. Experimental procedures

\begin{tabular}{|l|l|l|l|l|l|}
\hline $\begin{array}{l}\text { Baseline/Quiet } \\
\text { respiration }\end{array}$ & $\begin{array}{l}\text { Vocal reading } \\
\text { task 1 (5 min) }\end{array}$ & Recovery 1 & $\begin{array}{l}\text { Baseline/Quiet } \\
\text { respiration }\end{array}$ & $\begin{array}{l}\text { Vocal reading } \\
\text { task 2 (5 min) }\end{array}$ & Recovery 2 \\
\hline
\end{tabular}

Following the gas exchange experimental protocol, participants were asked to retrospectively rate their perceived vocal effort/ratings of perceived exertion for each of the reading tasks using the Borg scale (Borg, 1982; Appendix E). The choice to obtain these ratings after both vocal tasks had been completed was two-fold. First, obtaining the measure after each task might affect oxygen consumption during recovery, in turn affecting the recovery time for the preceding vocal task. Second, asking for ratings of perceived vocal effort/ perceived exertion during the actual protocol might reveal information about the purpose of the study and as noted the idea was to keep the intent of measuring vocal fatigue unknown to participants until after the protocol was completed.

Following the gas exchange protocol, participants were asked to complete a vocal efficiency test and a Bench Stepping Test. For the vocal efficiency test, all participants were asked to produce a standardized repeated consonant-vowel (/pi/) string at comfortable pitch and loudness using the PAS6600 mask (Holmberg, Hillman, \& Perkell, 1988); (dB SPL/cm H2O x cc/sec). Each participant produced three repetitions of 5 strings of /pi/. This measure was used as a gross estimate of the subject's vocal efficiency in general (Van den Berg, 1958; Grillo \& Verdolini, 2010) as further discussed in the Results section. After vocal efficiency data were recorded, all participants completed the Bench Stepping Test to obtain an estimate of overall cardiovascular fitness (Kasch, \& Boyer, 1968). For this test, participants were asked to step up onto and down from a 12-inch bench at 96 beats per minute for 3 minutes. Immediately at task 
completion, heart rate was recorded manually by recording the pulse rate. The Bench Stepping Test has been widely used in the exercise physiology literature to estimate cardiovascular fitness and has been shown to be a good correlate of VO2 max using instrumented measures (Jacks, Moore, Topp, \& Bibeau, 2008; Kasch, Phillips, Ross, Carter, \& Boyer, 1966).

\subsection{DATA REDUCTION}

Primary dependent variables including gross metabolic cost for reading and metabolic recovery from reading were extracted for further statistical analysis. A detailed description of data extraction for gross metabolic cost and metabolic recovery is provided below. Additionally, data extraction for the exploratory variables of S.A.1 (oxygen uptake and recovery kinetics) is described. Data extraction for secondary variables including ratings of perceived exertion, heart rate and vocal efficiency is also described.

\subsubsection{Gross metabolic cost for reading}

Metabolic cost can be obtained in one of two ways: (a) gross metabolic cost (which does not account for baseline oxygen consumption) and (b) net metabolic cost (in which oxygen consumption during a task is subtracted from baseline consumption). Gross metabolic cost was obtained in this study for two main reasons explained in the exercise physiology literature: (a) baseline oxygen consumption (in quiet breathing) is variable within individuals and is not a true representation of baseline metabolic rate, and (b) gross metabolic cost is more reproducible than net metabolic cost (Brehm, Knol, \& Harlaar, 2008). 
Gross metabolic cost $(\mathrm{ml} / \mathrm{kg}-\mathrm{w})$ was standardized to the rate of speech for each participant and was defined as the ratio of energy expenditure for reading $(\mathrm{ml} / \mathrm{kg} / \mathrm{min}) /$ rate of speech, where rate of speech was calculated as words per minute - wpm. Energy expenditure for the task was defined as the average of oxygen consumption from the end of the task to oneminute after task completion. The latter time point was selected to represent the energy expenditure for the task because: (a) for many individuals in the VF and VH groups, the instrument did not capture or record a breath or recorded very few breaths on expiration during the reading task, and (b) oxygen consumption continued to increase during the minute after the reading task, indicating that participants tended to pay for the task after the task had been completed (Mc Cardle et al., 2007; Waters et al., 1983). One of the reasons for the lack of or minimal recorded breaths during the task could be attributable to "possible breath holding" during reading by participants resulting in minimal airflow on expiration (Gillespie, GartnerSchmidt, Rubinstein, \& Verdolini-Abbott, 2013) and the inability of the instrument to record a breath on expiration.

\subsubsection{Extraction of reading metabolic recovery data}

Recovery data were extracted in two different ways. First, recovery time was calculated as time (sec) taken to reach baseline oxygen consumption values (within .2ml/kg/min) post task. The time was calculated following energy expenditure for reading (i.e., recovery time was calculated starting from post 1-min of reading). Second, 50\% recovery time was calculated as the number (percent) of individuals in a group who achieved 50\% recovery values at one- and two-minute intervals post task completion. This latter data point was calculated by obtaining the change in oxygen consumption from baseline to consumption during reading. Then $50 \%$ of that change was 
calculated and subtracted from the energy expenditure for reading. The calculated oxygen consumption then was compared to oxygen consumption during recovery at one-minute and twominute intervals to identify the number of individuals in each group who achieved $50 \%$ recovery at those time points (Scrutinio, Passantino, Lagioia, Napoli, Ricci, \& Rizzon, 1998; Cohen-Solal, Laperche, Morvan, Geneves, Caviezel, \& Gourgon, 1995).

\subsubsection{Descriptive exploration of raw data (Supplement to S.A. 1)}

Each individual participant's raw data were explored descriptively to gain insight in to pattern of oxygen use at the onset of reading and recovery from reading across the three groups. Raw data of oxygen consumption (ml/kg/min) were explored during the task and also following the task to understand the phenomenon of oxygen kinetics and excess post-exercise oxygen consumption (EPOC) respectively. Oxygen uptake increases exponentially at the onset of exercise and continues to increase until a steady state in oxygen uptake is reached. The dynamic behavior of oxygen uptake in the transition from rest to exercise is termed oxygen kinetics (Poole, Barstow, McDonough, \& Jones, 2008). To investigate oxygen kinetics, the data were visually inspected for the rise time from baseline to the plateau in oxygen uptake during the reading task. This variable may provide insight into the phenomenon of fatigue, specifically, neuromuscular inefficiency (Kemps et al., 2010).

Oxygen consumption was also inspected for EPOC. EPOC is defined as the excess oxygen consumed immediately following completion of a task to replenish the resources expended during the task, including oxygen deficit incurred at the beginning of the task, adenosine triphosphate (ATP), phosphates, and other metabolic byproducts (Hill, Long, \& Lupton, 1924; Bahr \& Sejersted, 1991; Gaesser \& Brooks, 1984; McCardle, et al., 2001, 2007). 
In other words, oxygen consumed immediately post-task was visually inspected for a rise, a decrease or no change from the oxygen consumed during reading. Data on EPOC will provide insight into the cardiac output that aids in recovery from exercise - that is, around cardiovascular recovery issues (Cohen-Solal et al., 1995).

\subsubsection{Secondary factors}

For ratings of perceived exertion, participants were asked to circle their appropriate level of perceived effort on the Borg's scale of perceived exertion for each reading task and the resulting data were used for further analysis. For cardiovascular fitness, heart rate was determined for each participant following completion of the Bench Stepping Test and heart rate was manually recorded.

For vocal efficiency, the middle three tokens of each of three five-syllable consonant vowel sets were manually selected and examined for acceptable morphology. Specifically, data were inspected for pressure peaks' return to baseline during vowel production and airflow values' return to baseline during consonant production (Smitheran \& Hixon, 1981). Once deemed appropriate, values for average pressure, airflow, and speech intensity during those productions were manually recorded and used to calculate vocal efficiency ( $\mathrm{dBSPL} / \mathrm{cmH}_{2} \mathrm{O} \mathrm{x} \mathrm{l} / \mathrm{s}$ ). Of the 32 participants, data from 3 participants was not available for analysis due to technical glitch and loss of collected data, including one participant from the VH group and two from the CV group. 


\subsection{STATISTICAL DATA ANALYSIS}

Inferential statistics were performed on the primary variables addressed in Specific Aim 1. To understand gross metabolic cost for reading, a 2x3 mixed-model Analysis of Variance (ANOVA) was used. The between subject factor was group (VF, VH, and CV) and the within-subject factor was task loudness (conversational versus loud). A mixed-model ANOVA was also conducted for metabolic recovery (time in sec) from reading tasks, using the same within- and betweensubjects factors (Table 4-3). For both these tests, assumptions of sphericity, and homogeneity of variance were tested for the mixed repeated measures design. The assumption of sphericity was met for all interaction effects. The assumption of homogeneity of variance for the dependent variable (DV) was met across all groups for all analyses and hence no adjustment was required for all analyses. For 50\% recovery time, only descriptive data on the number of individuals achieving 50\% recovery at 1 and 2 minutes post energy expenditure were obtained. Statistical analyses were not conducted for this variable, in the interest of protecting from alpha inflation for the primary analyses of interest.

Table 4-3 Dependent and independent variables for specific aim 1

\begin{tabular}{|l|l|l|}
\hline Dependent Variable & Independent Variable 1 & Independent Variable 2 \\
\hline Metabolic cost & Group (VF, VH, CV) & Loudness \\
\hline Recovery Time & Group (VF, VH, CV) & Loudness \\
\hline
\end{tabular}

Data were also visually inspected for interaction using line graphs to clarify the direction of any interactions in gross metabolic consumption and recovery across groups and also to 
observe variability in the data within and across groups. Box plots were used to visually inspect main effects. The center line in each box plot represents the median and the whiskers represent the variability in data within 1.5 quartiles from the median. Circles represent the outliers that do not fit in the quartiles. For data descriptively analyzed to elaborate on oxygen kinetics, line graphs were obtained for visual inspection of the data. For all other Secondary Aims 1-4, Pearson's product moment correlations were used to identify relationships between the primary (metabolic cost and recovery time) and secondary variables (perceived exertion of vocal effort, vocal efficiency, cardiovascular fitness and factors within VFI). For all analyses, the alpha level was set at $\mathrm{p}<.05$ without protection for alpha inflation due to the preliminary nature of the study. All statistical analyses were conducted using SPSS 21.0 software. 


\subsection{RESULTS}

\subsection{GROSS METABOLIC COST FOR READING}

Specific Aim 1: To identify gross metabolic cost during a vocal task and (b) to evaluate metabolic recovery times following task performance in individuals with vocal fatigue, in comparison to two control groups: (i) vocally healthy, vocally untrained, cardiovascular untrained individuals, and (ii) cardiovascular trained, vocally healthy individuals without voice training.

In terms of group comparison, descriptively, the gross metabolic cost for reading appeared similar across VF and VH groups (Table 5-1 and Figure 5.2). However, somewhat counterintuitively, individuals in the $\mathrm{CV}$ group displayed a higher mean gross metabolic cost

during reading compared to the other two groups (Table 5-1 and Figure 5.2). In terms of loudness, gross metabolic cost was greater for loud reading compared to the conversational reading across groups (Figure 5.3). 
Table 5-1. Mean (SD) values of gross metabolic cost for two different loudness levels with their corresponding mean (SD) dB sound pressure level (SPL) across the three groups of participants (vocal fatigue, vocally healthy and cardiovascular trained).

\begin{tabular}{|l|l|l|l|}
\hline \multicolumn{1}{|c|}{ Task } & $\begin{array}{l}\text { Vocal Fatigue } \\
\text { Mean (SD), } \mathrm{n}=12\end{array}$ & $\begin{array}{l}\text { Vocally Healthy } \\
\text { Mean (SD), } \mathrm{n}=12\end{array}$ & $\begin{array}{l}\text { Cardiovascular } \\
\text { Trained } \\
\text { Mean (SD), } \mathrm{n}=8\end{array}$ \\
\hline Conversational reading \\
\hline dBSPL & $69.8(2.3)$ & $69.6(1.9)$ & $70.8(1.8)$ \\
\hline $\begin{array}{l}\text { Gross metabolic cost } \\
\text { (ml/kg-w) }\end{array}$ & $.0175(.006)$ & $.0183(.007)$ & $.0223(.006)$ \\
\hline Loud reading & \multicolumn{3}{|l|}{} \\
\hline \multicolumn{1}{|c|}{$\mathrm{dBSPL}$} & $82.7(2.6)$ & $82.7(2.4)$ & $84.3(2.4)$ \\
\hline $\begin{array}{l}\text { Gross metabolic cost } \\
\text { (ml/kg-w) }\end{array}$ & $.0231(.009)$ & $.0221(.009)$ & $.0310(.007)$ \\
\hline
\end{tabular}

Statistical analysis using a mixed-model ANOVA was performed to assess the influence of group (VF, VH and $\mathrm{CV}$ ) and task (conversational and loud reading) on gross metabolic cost. Results were significant for a group $\mathrm{x}$ task interaction $(\mathrm{F}[2,29]=3.486, \mathrm{p}<.05$; Figure 5.1$)$. Numerically, the CV group had greater metabolic cost during reading in general, and further had a statistically verified increase in metabolic cost compared to the other groups.

There was no significant main effect for group $(\mathrm{F}[2,29]=2.364, \mathrm{p}<.05)$. The main effect of task loudness was significant $(\mathrm{F}[1,29]=63.577, \mathrm{p}<.001)$. 


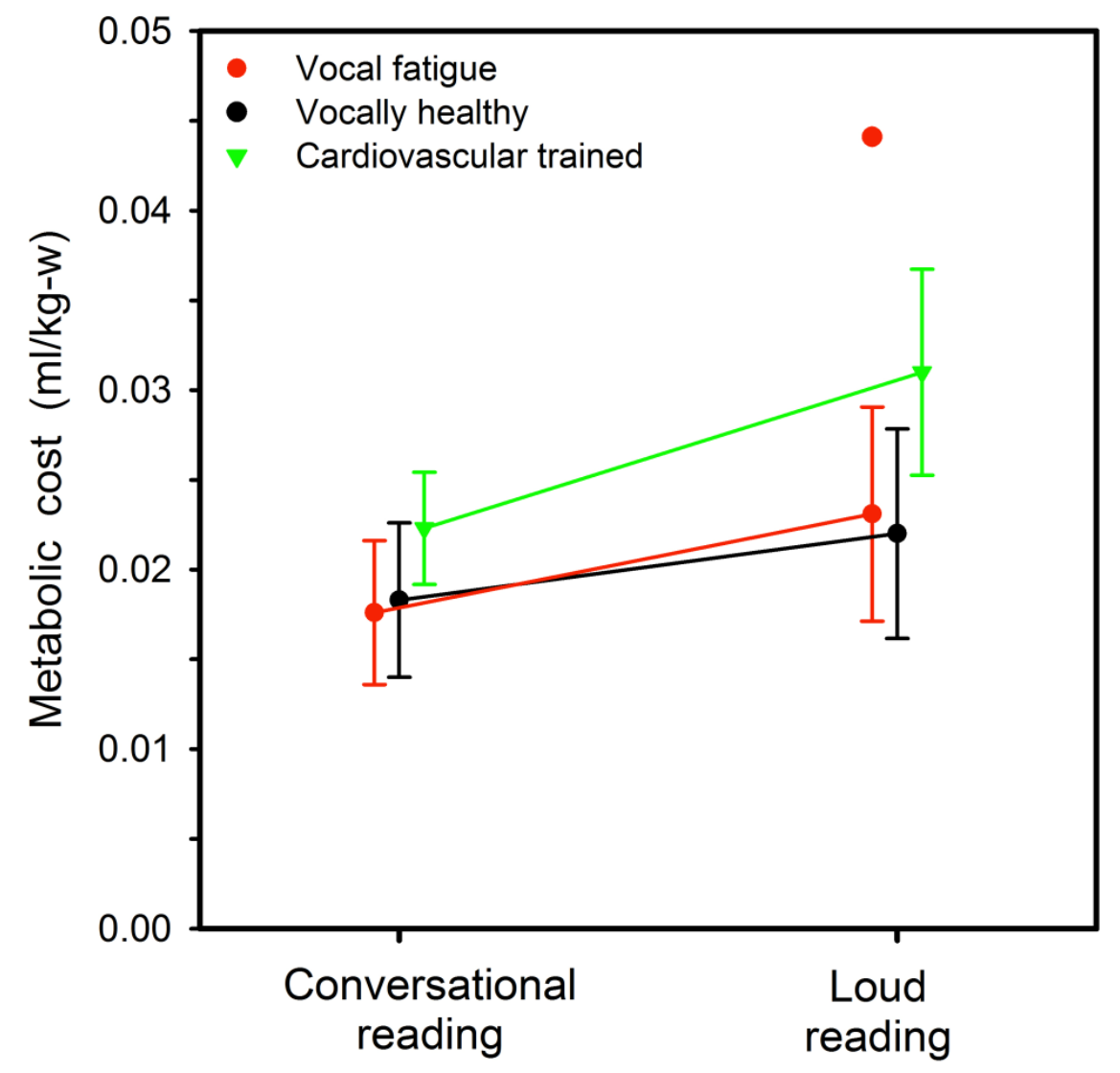

Figure 5-1 Metabolic cost (ml/kg-w) for conversational and loud reading across the three groups; vocal fatigue, vocally healthy and cardiovascular trained. 


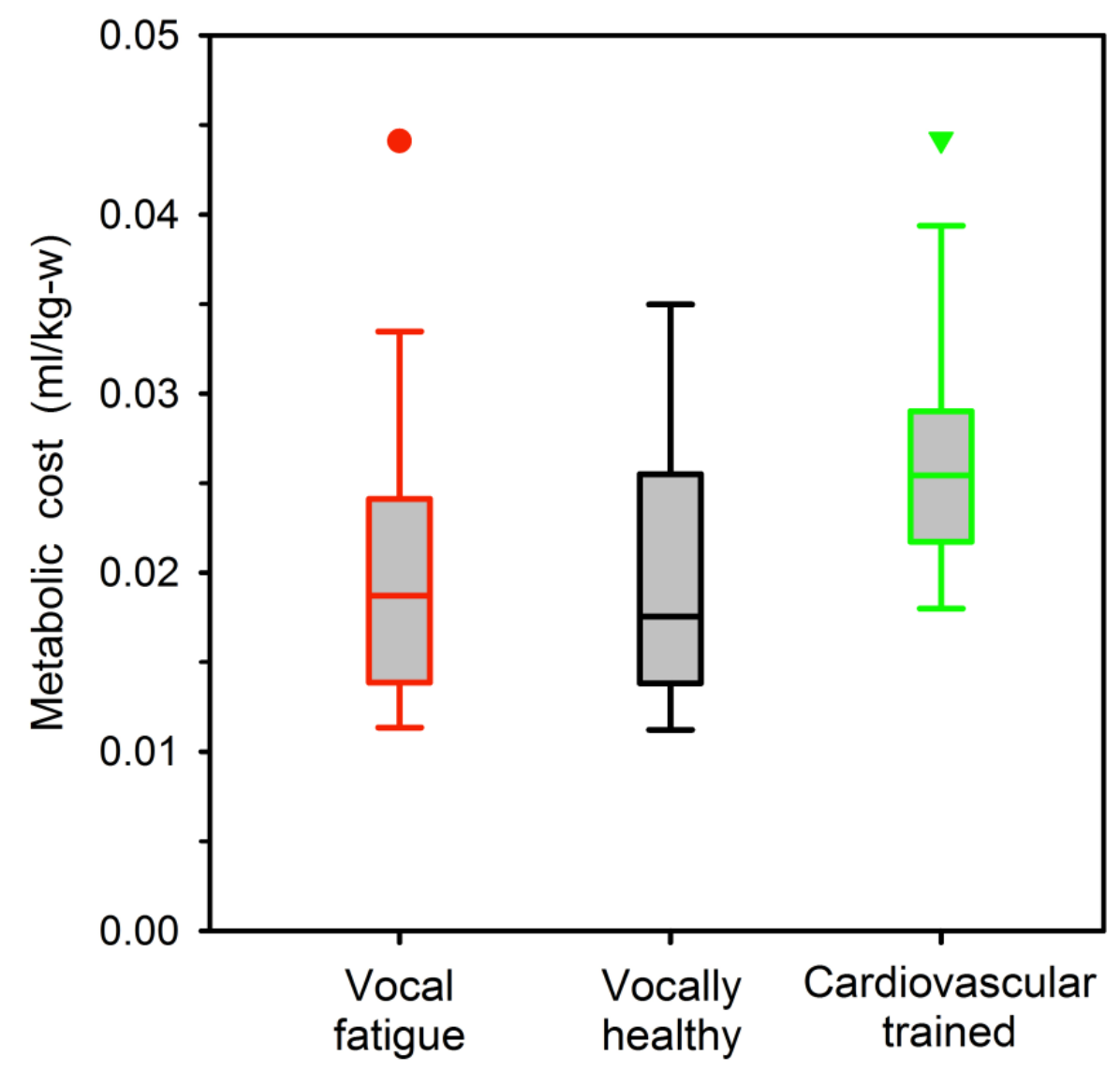

Figure 5.2. Metabolic cost (ml/kg-w) across the three groups; vocal fatigue, vocally healthy and cardiovascular trained collapsed across loudness conditions. 


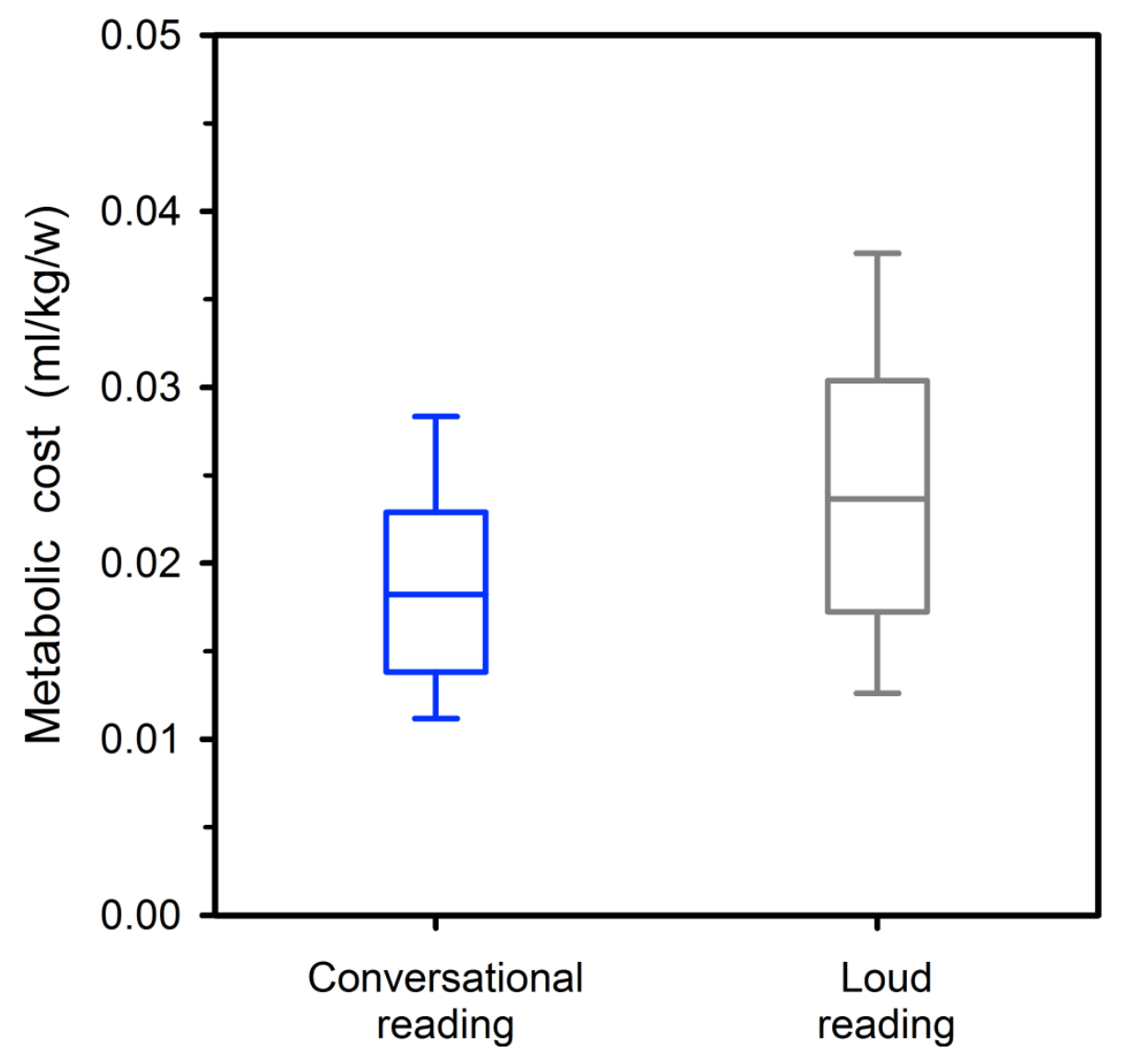

Figure 5.3. Average metabolic cost ( $\mathrm{ml} / \mathrm{kg}$-w) and variability for conversational and loud reading collapsed across groups.

\subsection{RECOVERY FROM READING}

Specific Aim 1: To identify gross metabolic cost during a vocal task and (b) to evaluate metabolic recovery times following task performance in individuals with vocal fatigue, in comparison to two control groups: (i) vocally healthy, vocally untrained, cardiovascular untrained individuals, and (ii) cardiovascular trained, vocally healthy individuals without voice training. 
Descriptive and statistical analyses are provided for the two-recovery data sets, including (a) recovery time, the time taken to reach baseline post energy expenditure for reading and (b) 50\% recovery time - number (percent) of individuals in each group returning to $50 \%$ oxygen consumption values 1 and 2 minutes following reading.

\subsubsection{Recovery time}

VH group had numerically greater mean recovery time and increased variability in recovery time for conversational reading compared to other two groups (Table 5.2, Figure 5.4). However, VF group showed longer recovery time and greater recovery variability for loud reading (Table 5.2). Additionally, especially for loud reading, variability in recovery time was patently least for the CV group. Descriptively, recovery time was longer for loud reading compared to conversational reading across all three groups (Table 5.2, Figure 5.6).

Table 5.2. Descriptive Data (Mean, SD) for Conversational and Loud Reading Recovery Time in Seconds for the Three Groups (Vocal Fatigue, Vocally Healthy and Cardiovascular Trained Individuals).

\begin{tabular}{|l|l|l|l|}
\hline Task/Group & $\begin{array}{l}\text { Vocal Fatigue } \\
\text { Mean (SD), n=12 }\end{array}$ & $\begin{array}{l}\text { Vocally Healthy } \\
\text { Mean (SD), n=12 }\end{array}$ & $\begin{array}{l}\text { Cardiovascular } \\
\text { Trained } \\
\text { Mean (SD), } \mathrm{n}=8\end{array}$ \\
\hline $\begin{array}{l}\text { Recovery time- } \\
\text { Conversational } \\
\text { reading (secs) }\end{array}$ & $100(63.37)$ & $107.75(82.28)$ & $99.53(64.76)$ \\
\hline $\begin{array}{l}\text { Recovery time- } \\
\text { Loud reading (secs) }\end{array}$ & $174.5(91.43)$ & $140.58(78.08)$ & $131.75(36.92)$ \\
\hline
\end{tabular}

Statistical analysis using mixed ANOVA revealed no significance for group $\mathrm{x}$ task interaction $(F[2,29]=.647, \mathrm{p}>.05)$, (Figure 5.4). Similarly, there was no significant main effect 
for group and all groups had similar recovery times $(\mathrm{F}[2,29]=.634, \mathrm{p}>.05)$. However, the main effect of loudness was significant $(F[1,29]=9.528, \mathrm{p}<.005)$.

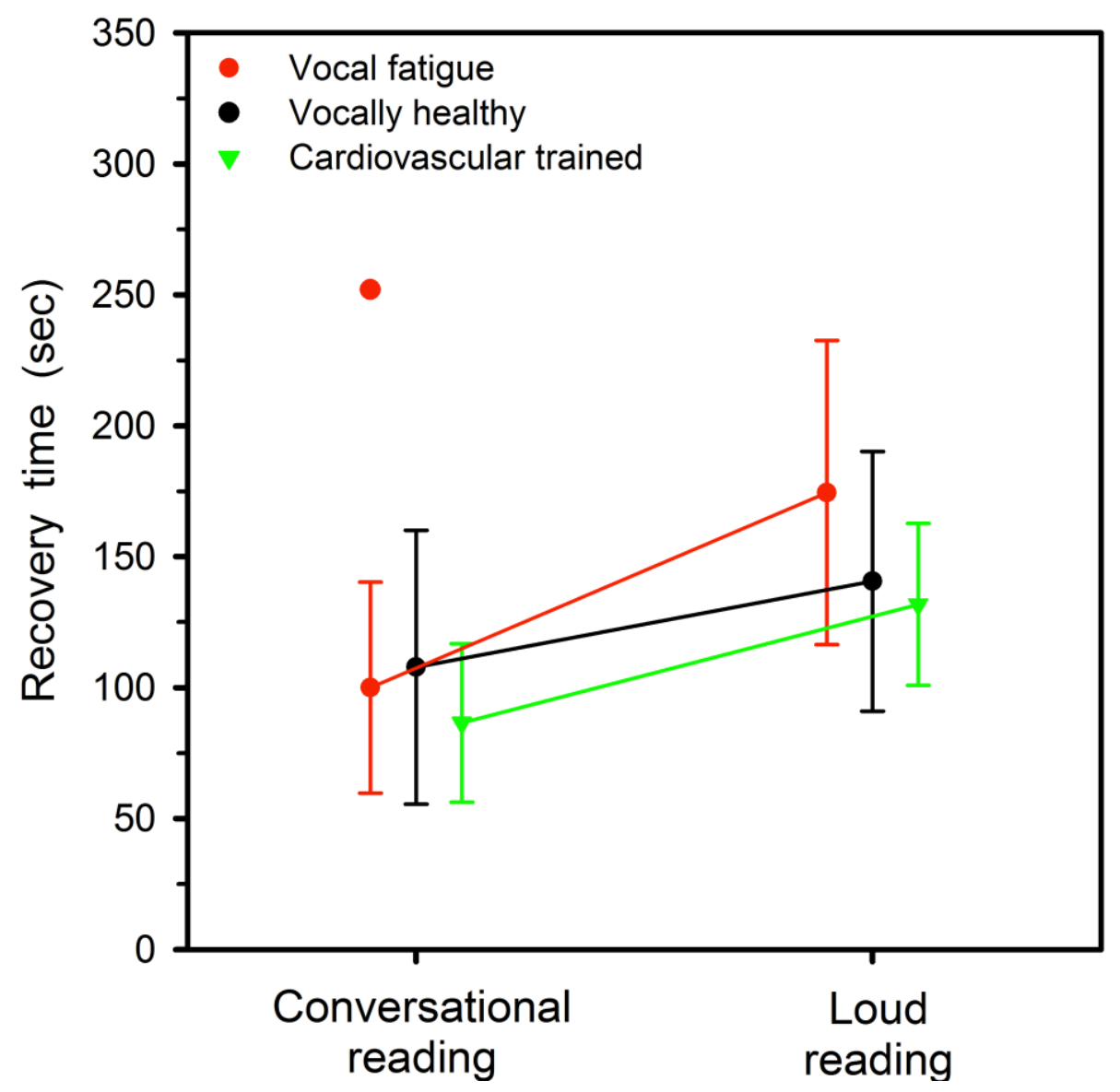

Figure 5.4. Average and variability of recovery time (sec) for conversational and loud reading across the three groups; vocal fatigue, vocally healthy and cardiovascular trained. 


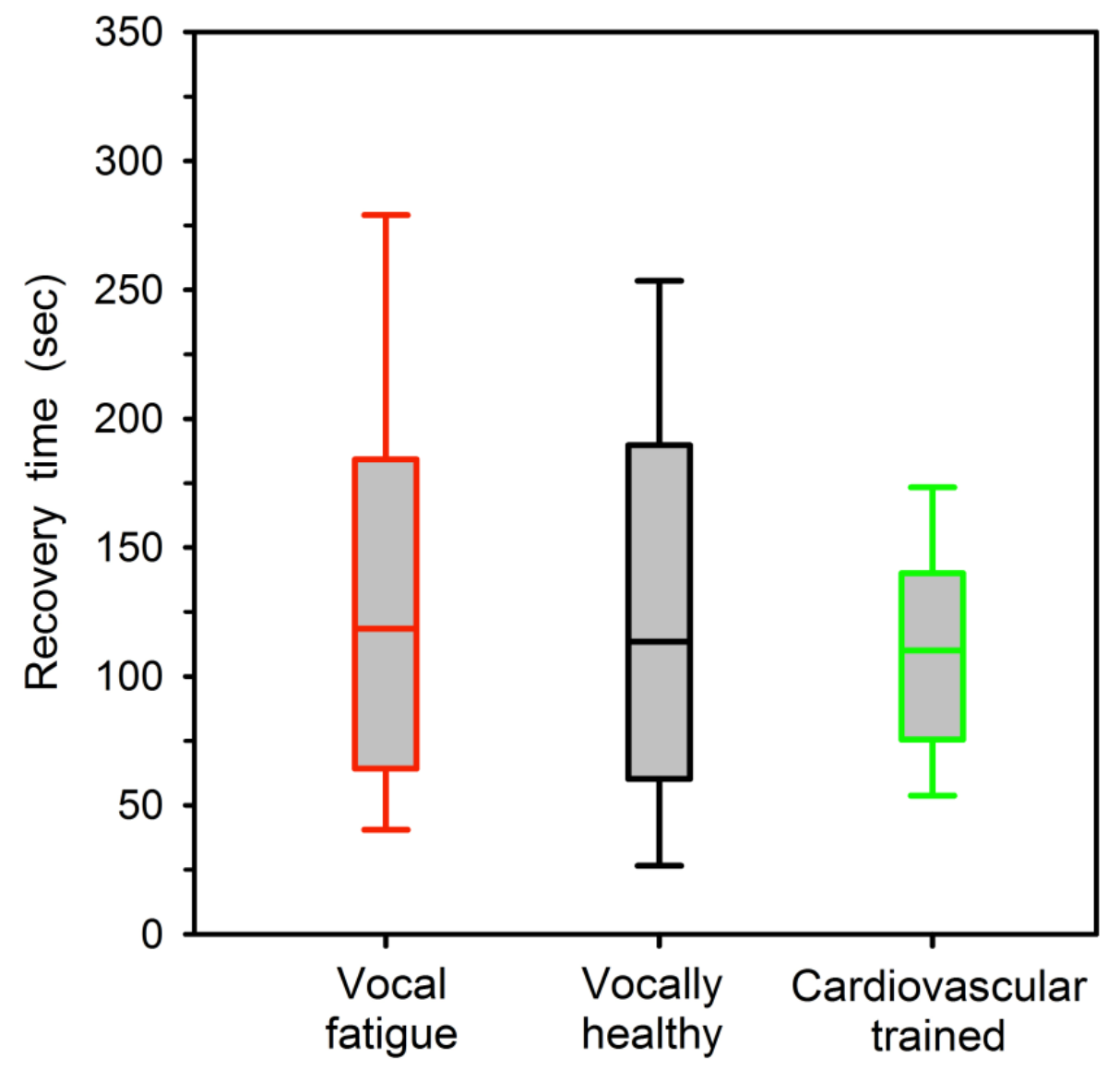

Figure 5-5. Average variability in recovery time (sec) for the three groups- vocal fatigue, vocally healthy and cardiovascular trained, collapsed across loudness conditions. 


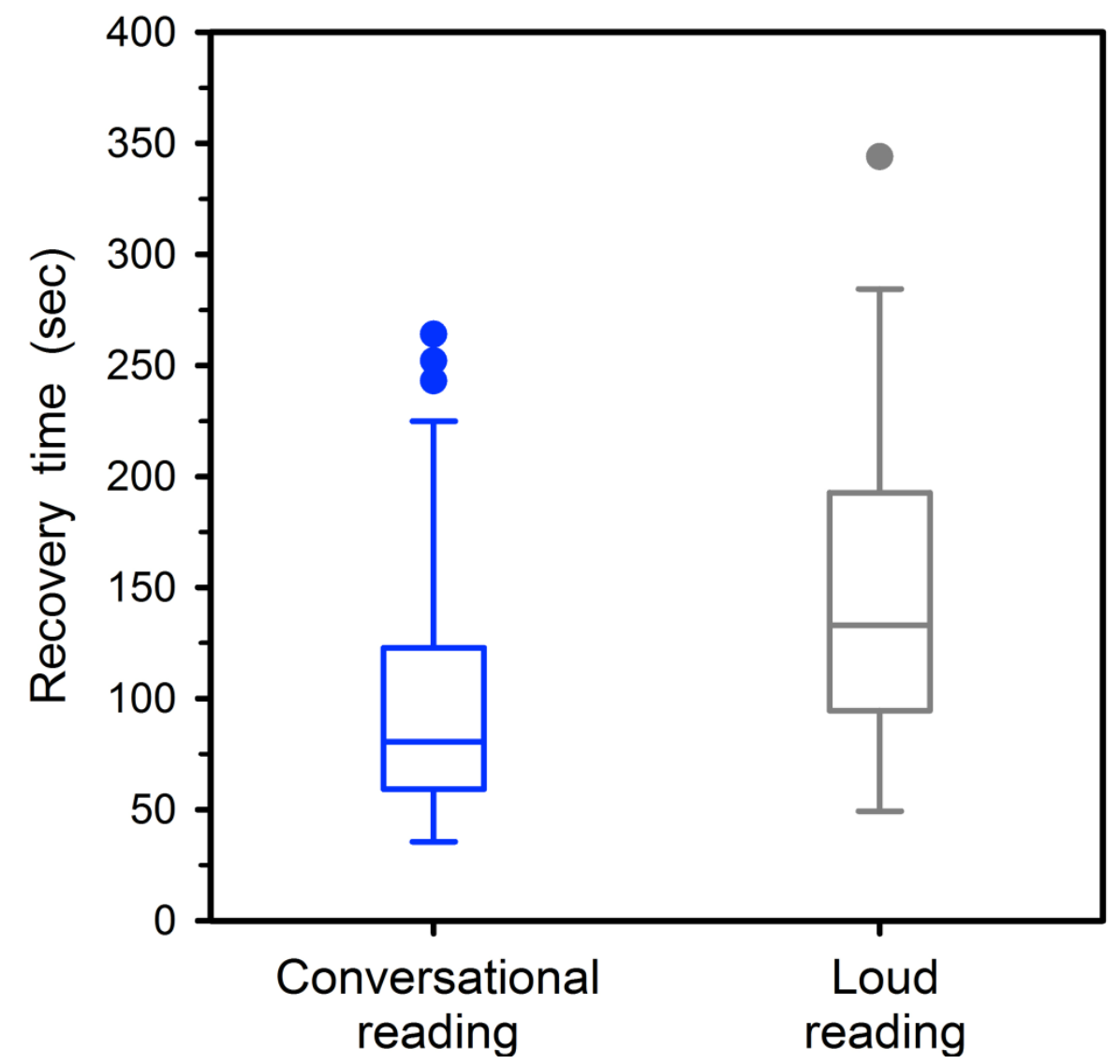

Figure 5-6. Average and variability of recovery time (sec) for conversational and loud reading, collapsed across groups.

\section{$5.2 .250 \%$ recovery time}

As indicated, another indicator of recovery from the reading task assessed the number of individuals (percentage) in each group obtaining 50\% recovery in oxygen consumption at one and two-min post energy expenditure for reading. For conversational reading, descriptively, there was minimal difference in this number across groups. However, for loud reading, 62.5\% of individuals in the $\mathrm{CV}$ group reached $50 \%$ recovery level by one-minute post reading compared to only $25 \%$ of individuals in the $\mathrm{VH}$ and VF groups (Table 5.3). Oddly, but 
surprisingly, VF group were similar to CV group at 2 - minute time point for loud reading. No statistical analysis was performed for this recovery data extraction.

Table 5.3. Distribution of $\mathbf{5 0 \%}$ Recovery at One and Two Minutes Post Energy Expenditure for Reading by Group (n, \%)

\begin{tabular}{|c|c|c|c|}
\hline & $\begin{array}{c}\text { Vocal Fatigue } \\
n=12\end{array}$ & $\begin{array}{l}\text { Vocally Healthy } \\
n=12\end{array}$ & $\begin{array}{c}\text { Cardiovascular } \\
\text { Trained, } n=8\end{array}$ \\
\hline \multicolumn{4}{|l|}{ Conversational reading } \\
\hline $\begin{array}{l}\text { 1-minute } \\
\text { 50\% recovery, n (\%) }\end{array}$ & $7(58)$ & $6(50)$ & $4(50)$ \\
\hline $\begin{array}{l}\text { 2-minute } \\
\text { 50\% recovery, n (\%) }\end{array}$ & $10(83)$ & $9(75)$ & $7(88)$ \\
\hline \multicolumn{4}{|l|}{ Loud reading } \\
\hline $\begin{array}{l}\text { 1-minute } \\
\text { 50\% recovery, n (\%) }\end{array}$ & $3(25)$ & $3(25)$ & $5(62.5)$ \\
\hline $\begin{array}{l}\text { 2-minute } \\
50 \% \text { recovery, n (\%) }\end{array}$ & $11(92)$ & $9(75)$ & $7(88)$ \\
\hline
\end{tabular}

\subsection{DESCRIPTIVE EXPLORATIONS}

Supplement to S A 1: To explore oxygen uptake kinetics and recovery kinetics (EPOC) in individuals with vocal fatigue in comparison to two non-fatigue groups.

\subsubsection{Oxygen kinetics}

Line graphs were drawn for all participants whose air exchange signals on expiration were captured during conversational and loud reading. Representative and meaningful data displays 
are shown in Figures 5.7-5.10. For three participants in the VF group and four participants in the VH group, breath-by-breath gas exchange data on expiration were not captured during reading, likely due to minimum expiratory airflow during reading. However, signals were captured for every participant in the CV group. Further, all individuals in the CV group consistently showed a rise in oxygen uptake at the onset of the reading task and reached a plateau within 2-3 minutes after task onset, for both reading tasks (e.g., Figure 5.7). However, the VH group showed variable patterns including achieving a plateau in oxygen uptake, or consistent rise in oxygen uptake with no plateau. Further, one individual in the VH group showed a double rise time and double plateau during the task (Figure 5.8). With one exception, individuals with VF showed either no change in oxygen uptake from baseline through end of task performance (Figure 5.9) or a consistent rise in oxygen uptake until the end of the reading task with no plateau (Figure 5.10).

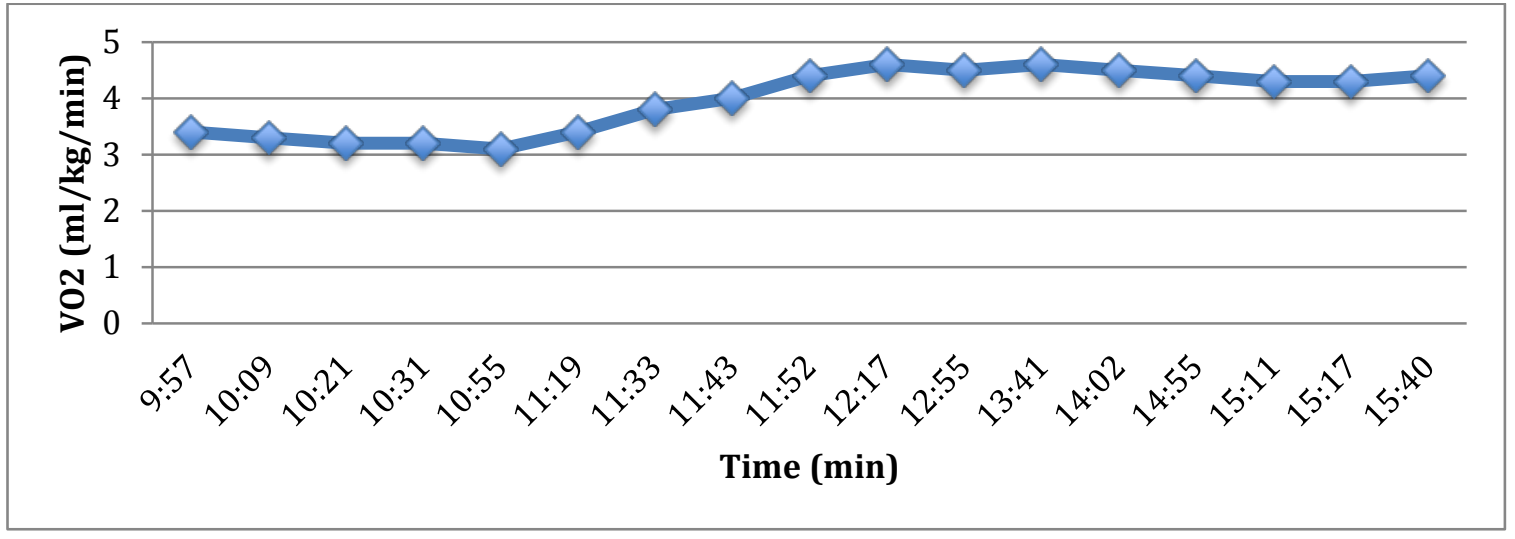

Figure 5-7. Line graph demonstrating typical pattern of oxygen kinetics in cardiovascular trained individuals - with rise time starting at $\mathbf{1 0 . 5 5}$ at the onset of reading task and a plateau achieved at 12.17 during loud reading. 




1:25 1:42 1:52 2:02 2:13 2:23 2:43 3:03 3:23 3:43 4:03 4:23 4:43 5:03 5:23 5:43 6:03 6:23 6:43

Time (min)

Figure 5-8. Line graph demonstrating atypical oxygen kinetics indicating a double plateau in a vocally healthy individual (VH8). The $1^{\text {st }}$ rise time is at 2.02 at the onset of reading and $1^{\text {st }}$ plateau achieved at 3.03 , followed by a $2^{\text {nd }}$ rise time at 4.43 during the task and $2^{\text {nd }}$ plateau achieved at 5.43 .

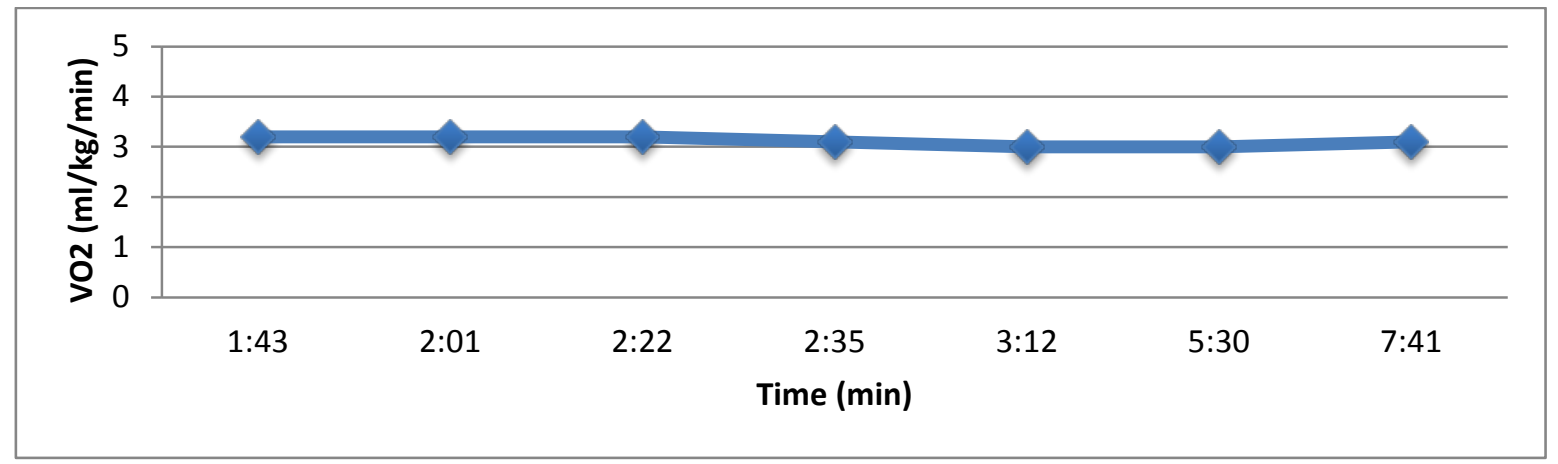

Figure 5-9. Line graph illustrating one pattern of oxygen kinetics observed in six individuals with vocal fatigue (e.g., VF12) showing no change in oxygen consumption from baseline to task performance.

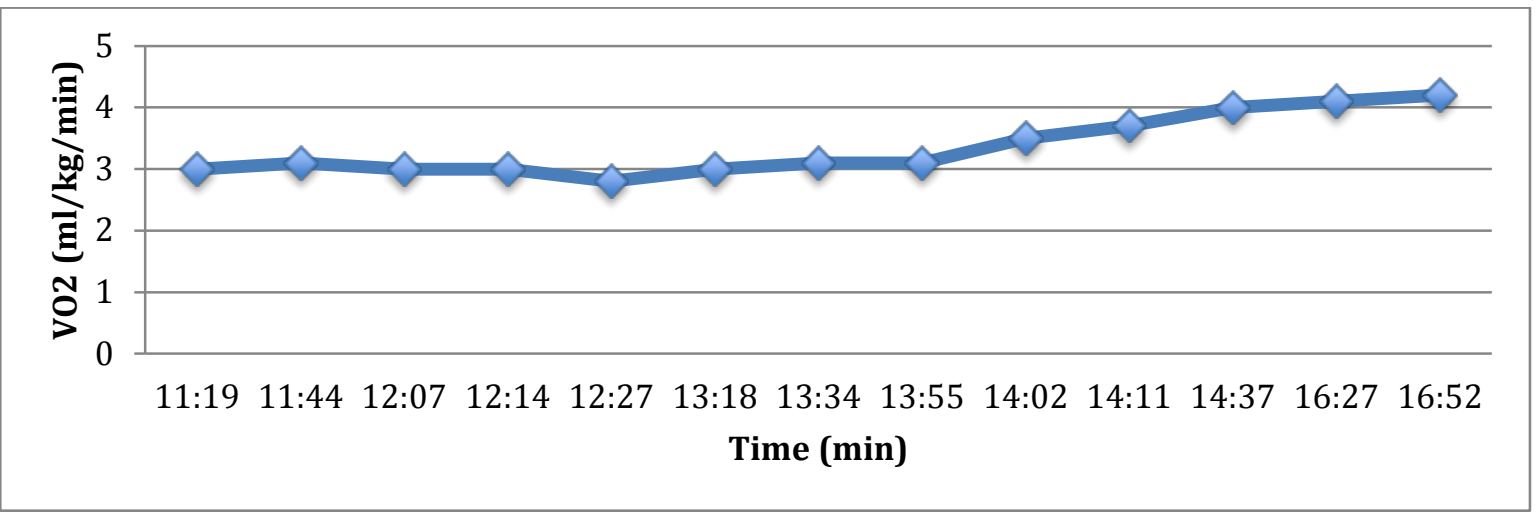

Figure 5-10. Line graph illustrating a second pattern of oxygen kinetics observed in other individuals with vocal fatigue (e.g, VF7) with rise time starting at 12.27 and no plateau achieved during the task. 


\subsubsection{Excess post-exercise oxygen consumption (EPOC)}

Post reading oxygen consumption was inspected to infer whether a rise, a drop or no change from oxygen consumption occurred during reading. Descriptive data on the number of individuals who showed an increase in oxygen consumption (>.2ml/kg/min) post reading across the three groups for each task is shown in Table 5.4.

Table 5.4. Number (Percent) of Individuals with a Rise in Oxygen Consumption (ml/kg/min) Post Reading (EPOC) across the Three Groups.

\begin{tabular}{|l|c|c|c|}
\hline Task/Group & $\begin{array}{c}\text { Vocal Fatigue } \\
\mathrm{n}=12\end{array}$ & $\begin{array}{c}\text { Vocally Healthy } \\
\mathrm{n}=12\end{array}$ & $\begin{array}{c}\text { Cardiovascular } \\
\text { Trained } \\
\mathrm{n}=8\end{array}$ \\
\hline $\begin{array}{l}\text { Conversational } \\
\text { reading, } \mathrm{n}(\%)\end{array}$ & $8(67)$ & $8(67)$ & $3(37)$ \\
\hline Loud reading, $\mathrm{n}(\%)$ & $9(75)$ & $8(67)$ & $3(37)$ \\
\hline
\end{tabular}

\subsection{SECONDARY AIMS}

For all secondary aims, Pearson’s correlational analyses were performed. 


\subsubsection{Secondary Aim 1}

To determine perceived phonatory effort for the vocal tasks and to determine if a correlation exists between perceived phonatory effort/perceived exertion using the Borg scale and gross metabolic cost for the vocal tasks.

A first observation regarding RPE themselves: for conversational reading, mean ratings of perceived effort (measured by ratings of perceived exertion; RPE 6-20) were greatest for the VF group and was the least for CV group. For loud reading, ratings of perceived effort was similar for VH and VF groups and again the least for the CV group (Table 5.5).

Table 5.5. Descriptive Data for Perceived Effort (RPE) for both Conversational and Loud Reading across the Three Groups (Vocal Fatigue, Vocally Healthy, and Cardiovascular Trained).

\begin{tabular}{|l|l|l|l|}
\hline Task/Group & $\begin{array}{l}\text { Vocal Fatigue } \\
\text { Mean (SD), } \mathrm{n}=12\end{array}$ & $\begin{array}{l}\text { Vocally Healthy } \\
\text { Mean (SD), } \mathrm{n}=12\end{array}$ & $\begin{array}{l}\text { Cardiovascular } \\
\text { Trained } \\
\text { Mean (SD), } \mathrm{n}=8\end{array}$ \\
\hline Conv RPE, 6-20 & $9.1(1.5)$ & $7.8(1.9)$ & $6.9(1.2)$ \\
\hline Loud RPE, 6-20 & $15.6(2.3)$ & $15.1(2.4)$ & $11.6(2)$ \\
\hline
\end{tabular}

Pearson's correlation between metabolic cost and perceived effort for conversational loudness was $r=.044(p>.05)$ collapsed across the three groups. Correlation between metabolic cost and perceived effort for loud reading was $r=-.126(p>.05)$ pooled across the three groups.

\subsubsection{Secondary Aim 2}

To determine heart rate as a measure of cardiovascular fitness and if such measure correlates with recovery time following the vocal task. 
Mean heart rate obtained following the Bench Stepping Test was highest for the VH group. There was minimal difference in heart rate between VF and CV groups. However, interestingly, the CV group demonstrated minimal variability across subjects in this measure, the VF group showed the greatest inter-subject variability and two subjects in that group failed to complete the test at all (Table 5.6). Pooling across subjects, results showed a poor correlation between Bench Stepping Test results and recovery time for both conversational reading $(r=.01$, $\mathrm{p}>.05)$ and loud reading $(\mathrm{r}=.14, \mathrm{p}>.05)$. In difference to predictions, heart rate recovery did not predict shorter or longer recovery time.

Table 5.6 Descriptive Data (Mean, SD) for Heart Rate across the Three Groups (Vocal Fatigue, Vocally Healthy, and Cardiovascular Trained).

\begin{tabular}{|c|c|c|}
\hline $\begin{array}{c}\text { Vocal Fatigue } \\
\text { Mean (SD), } \mathrm{n}=10\end{array}$ & $\begin{array}{c}\text { Vocally Healthy } \\
\text { Mean (SD), } \mathrm{n}=12\end{array}$ & $\begin{array}{c}\text { Cardiovascular Trained } \\
\text { Mean (SD), } \mathrm{n}=8\end{array}$ \\
\hline $83.4(18.9)$ & $95.2(15.8)$ & $85.3(8)$ \\
\hline
\end{tabular}

\subsubsection{Secondary Aim 3}

To determine vocal efficiency of the three groups and if measures of vocal efficiency correlate with gross metabolic cost for the vocal task.

Average vocal efficiency (dBSPL/cmH20 x l/sec) was calculated for each group. Calculations were manually obtained using the formula. Results revealed that numerically, vocal efficiency was highly variable within and across the three groups (Table 5.7). Further, one individual in the VF group displayed an unusually high efficiency value (efficiency value of 443 $\mathrm{dB} / \mathrm{cmH}_{2} 0 \times \mathrm{l} / \mathrm{s}$ ). Efficiency was recalculated for that group without the outlier (Table 5.8). 
Somewhat oddly, and contrary to expectations, even after the recalculation, it appeared that efficiency was actually better in the VF group than in the VH group, although it was best in the CV group.

Table 5.7. Descriptive Data (Mean and SD) for Vocal Efficiency $\left(\mathrm{dB} / \mathrm{cmH}_{2} \mathrm{O} \times \mathrm{l} / \mathrm{s}\right)$ across Three Groups (Vocal Fatigue, Vocally Healthy, and Cardiovascular Trained).

\begin{tabular}{|l|l|l|}
\hline $\begin{array}{l}\text { Vocal Fatigue } \\
\text { Mean (SD), } \mathrm{n}=12\end{array}$ & $\begin{array}{l}\text { Vocally Healthy } \\
\text { Mean (SD), } \mathrm{n}=11\end{array}$ & $\begin{array}{c}\text { Cardiovascular } \\
\text { Trained } \\
\text { Mean (SD), } \mathrm{n}=6\end{array}$ \\
\hline $123.59(113.1)$ & $85.68(48.9)$ & $108.46(55.9)$ \\
\hline
\end{tabular}

Table 5.8. Descriptive Data (Mean and SD) for Vocal Efficiency $\left(\mathrm{dB} / \mathrm{cmH}_{2} \mathrm{O} \times \mathrm{l} / \mathrm{s}\right)$ across Three Groups removing Outlier in the Vocal Fatigue Group (Vocally Healthy, Vocal Fatigue, and Cardiovascular Trained)

\begin{tabular}{|c|c|c|}
\hline $\begin{array}{c}\text { Vocal Fatigue } \\
\text { Mean (SD), } \mathrm{n}=11\end{array}$ & $\begin{array}{c}\text { Vocally Healthy } \\
\text { Mean (SD), } \mathrm{n}=11\end{array}$ & $\begin{array}{c}\text { Cardiovascular Trained } \\
\text { Mean (SD), } \mathrm{n}=6\end{array}$ \\
\hline $94.53(54.1)$ & $85.68(48.9)$ & $108.46(55.9)$ \\
\hline
\end{tabular}

Pooled across three groups, correlations between gross metabolic cost and vocal efficiency were positive and significant $r=.37(\mathrm{p}<.05)$, and $\mathrm{r}=.47(\mathrm{p}<.01)$ for conversational and loud voice, respectively. However, when the outlier was removed, correlations dropped to $.16(\mathrm{p}>.05)$ and $-.27(\mathrm{p}>.05)$ respectively.

\subsubsection{Secondary Aim 4}

To determine if correlations exist between Factor 1 of the VFI (avoidance of voice use and tiredness of voice) and metabolic cost during the vocal task and between Factor 3 of the VFI 
(improvement of voice symptoms or lack thereof with voice rest) and post-task recovery in the vocal fatigue group.

Overall, gross metabolic cost was positively related to self-reported voice avoidance and tiredness of voice use, VFI Factor $1(\mathrm{r}=.71, \mathrm{p}<.05)$ and $\mathrm{r}=.65, \mathrm{p}<.05$ for conversational and loud reading, respectively). This finding indicates that higher the VFI score on Factor 1, the greater the metabolic cost was in reading.

In contrast, numerically, as predicted recovery time was negatively correlated with selfreported improvement of voice symptoms with rest, VFI Factor 3. However, correlations were not statistically significant $(r=-.43, p>.05$ and $r=-.38, p>.05$ for conversational and loud reading). If these values were strengthened with a larger sample size, the implication is that the lower (poorer) the scores for VFI factor 3, the longer post-reading recovery would be expected to take and in fact, people's vocal fatigue symptoms would not get particularly better with rest. 


\subsection{DISCUSSION}

The purpose of the present study was to investigate potential metabolic mechanisms underlying vocal fatigue. The primary subjects of interest were individuals with vocal fatigue syndrome, without known organic pathology, as determined by the combination of results from a newly vetted questionnaire, the Vocal Fatigue Index (VFI), and laryngeal examination. The approach replicated methods in the exercise physiology domain using gas exchange analysis. Metabolic data including gross metabolic cost for reading and metabolic recovery time from reading were collected from subjects with vocal fatigue (VF), from vocally healthy subjects (VH), and from cardiovascular trained (CV) individuals. The study's original focus was two factors thought to underlie physical fatigue in general: (a) neuromuscular inefficiency as evidenced by increased metabolic cost during performance of a task and (b) cardiovascular recovery issues as evidenced by increased recovery time following task performance. The study's primary purpose was to determine if evidence for one or both mechanisms could be found in association with vocal fatigue. Key secondary analyses involved the examination of $\left(\mathrm{a}_{1}\right)$ oxygen uptake kinetics as a more finely grained window into neuromuscular efficiency during task performance (pattern of muscle use); ( $\left.b_{1}\right)$ percentage of individuals achieving 50\% recovery at 1 and 2 minutes post task, which might provide further insight into task recovery; and $\left(\mathrm{b}_{2}\right)$ post-task oxygen recovery (EPOC), which also might provide additional insight into recovery.

Primary expectations based on findings from the exercise physiology literature were that individuals with confirmed vocal fatigue syndrome would display (a) greater metabolic cost 
during a vocalization task (reading) compared to the other groups, and (b) slower post-task recovery time than other groups. In patent contrast to these expectations, neither of these effects was confirmed. Primary analyses failed to clearly identify any group differences in either metabolic cost during vocal task performance, or in post-task metabolic recovery time, let alone a differentiation of vocal fatigue versus control groups. The main finding of interest in these analyses was a group $*$ loudness interaction for metabolic cost during reading, showing that subjects in the CV group - had numerically greatest metabolic cost during both reading tasks compared to VH and VF groups. Another finding, which was not central to the main question of interest, regarded a main effect of loudness. Across groups, both metabolic costs during reading and recovery time following it increased with loud reading levels. Of itself, this latter effect was not particularly surprising.

In sum, mechanisms hypothesized to be relevant to vocal fatigue were not even vaguely captured in the present data set with respect to primary variables investigated. However, key secondary observations did reveal some insights.

\section{$\underline{\text { Secondary measures }}$}

In contrast to primary measures that were the original focus of the study, secondary "back-up” metabolic measures ended up providing potentially meaningful insights into vocal fatigue syndrome or at least protection from it. First is the case of oxygen uptake kinetics. These are defined as the dynamic behavior of oxygen uptake in the transition from rest to exercise (Poole et al., 2008). Such kinetics are dependent on two main mechanisms: (a) central circulation to deliver oxygen to working muscles, and (b) the ability of working muscles to 
extract oxygen from blood flow arriving to the muscles. In the present study, oxygen uptake patterns were strikingly different across VF, VH, and CV control groups. Individuals in the VF group showed either no change in oxygen uptake kinetics from baseline to reading, or showed a consistent rise in oxygen uptake kinetics at the onset of reading until the end of the task, without plateau. Individuals in the $\mathrm{VH}$ group showed an inconsistent pattern including achieving a plateau, a double plateau, or a consistent rise in uptake kinetics until the end of task, whereas individuals in the CV group showed consistent patterns of achieving plateau in oxygen uptake within 2-3 minutes of task onset. A possible explanation would be VF subjects' initial use of anaerobic energy sources for reading, in contrast to CV subjects' use of aerobic ones (Scott, 2005). A possible explanation for VH group would be presumably a combination of anaerobic and aerobic sources for reading varying based on the type of oxygen uptake kinetics pattern (e.g., plateau achieved- aerobic means; double plateau- anaerobic and aerobic energy). An important corollary is that the VF group's apparent reliance on anaerobic means at the onset of exercise would be predicted to create an oxygen deficit greater than occurred during CV's use of aerobic mechanisms, thus resulting in greater fatigue, defined as the increase in levels of oxygen uptake and delay or lag in achieving steady state (Poole et al., 1991; Reybrouck et al., 2007).

Second, in fact, findings for the number of subjects showing 50\% recovery at one minute post loud task completion were generally consistent with the foregoing speculations. A considerably smaller proportion of subjects in both VF and VH groups achieved 50\% recovery at one-minute post-task as compared to subjects in the CV group. The implication is that apparent anaerobic mechanisms most strikingly used by subjects in the VF group - but also to some extent in the VH group -- resulted in longer aerobic "pay-back" than required for subjects in the more aerobically trained CV group. 
Third, findings for post-task oxygen consumption (EPOC) were further consistent with the hypothesis that potential anaerobic energy use during task performance resulted in the need to "pay back" depleted resources with oxygen after task completion. Descriptively, subjects in both VF and VH groups demonstrated clearly greater post-task oxygen consumption than subjects in the CV group.

Fourth, average perceived effort was greater for subjects in VF and VH groups as compared to the CV group. Thus, perceived effort appeared to covary inversely with efficient oxygen uptake kinetics at the onset of the reading task.

In short, the primary story that the present data seem to tell is that poor oxygen uptake kinetic dynamics during task performance appear associated with an increased sense of vocal effort (fatigue), and lead to an increased need to "pay back" depleted oxygen resources post task. In contrast, more efficient oxygen uptake during reading appears associated with a decreased sense of effort (fatigue), and decreased post task aerobic "payback.” Stated differently, those in the CV group appeared to use an aerobic "pay as you go" approach to task performance somewhat paradoxically experiencing less effort during reading -- whereas those in VF and VH groups appeared to use incur oxygen deficit during task performance, increasing the sense of effort and creating debt that had to be paid back after the fact.

Somewhat disappointingly, none of the other secondary measures revealed any ability to distinguish the groups or to predict primary outcome measures in any interpretable way. Specifically, both vocal efficiency and heart rate recovery based on the Bench Stepping Test failed to distinguish the groups, and also failed to correlate with metabolic cost during task performance or recovery time, respectively, as predicted. Retrospectively, especially these latter results are unsurprising, as the primary outcome measures themselves did not distinguish the 
groups. Possibly, secondary measures might correlate with the more sensitive measures of oxygen uptake kinetics and EPOC. However, those analyses were not conducted as they were not pre-planned. Moreover, findings of a significant positive correlation between Factor 1 on the VFI (tiredness of voice, etc.) and metabolic costs during reading, and a "trending" negative relation between Factor 3 on the VFI (improvement in fatigue symptoms with rest) matched predictions and at that level were encouraging. Such data may point to differential treatment options for individuals with different metabolic profiles, but needs to be interpreted with caution at this point. The data appeared to contradict impressions from other secondary data implying an inverse relationship between perceived effort and metabolic cost, in particular. Given the complexity of the data set in general, for the time being the best approach will be to attribute this apparent contradiction to experimental artifact and set it aside for future inquiry rather than attempt to explain it presently.

Returning to apparent findings, it is somewhat perplexing that although descriptively, oxygen uptake kinetics, perceived reading effort, and EPOC appeared to distinguish individuals with vocal fatigue from cardiovascular trained individuals, with the possible exception of oxygen uptake, these measures failed to clearly distinguish those with vocal fatigue from putatively vocally healthy subjects. Potential explanations may lie with recruitment methods and subject characteristics in VF and VH groups. All subjects, including subjects in the VF group, were recruited through community flyers. Thus, subjects in the VF group were not individuals who self-referred to a clinic with clinically relevant complaints of vocal fatigue. Perhaps the recruitment of such individuals would increase the ability to differentiate findings for VF and VH groups. Moreover, the main differences between VF and VH groups resided not only with their VFI scores but also with occupational demographics. Ten of the 12 participants in the VF 
group were either teachers or students majoring in vocal music. In contrast, of the 12 participants in the vocally healthy group, only one participant was a school teacher, another was a vocal music major, and one more was a school-based speech-language pathologist. The remaining nine subjects in that group were undergraduate or graduate students who did not encounter heavy voice use on a regular basis. Potentially, some proportion of subjects in that group might experience vocal fatigue if voice use demands were increased, and thus their foundational profiles might be similar to those in the VF group. Thus, given the general lack of distinction between findings for VF and VH groups, in comparison to the CV group, in the end, results from this study may have ended up being as much about mechanisms that may protect from vocal fatigue as about mechanisms that may underlie it once it develops.

\section{$\underline{\text { Potential physiological mechanisms }}$}

At this point, we turn to tentative descriptions of physiological processes that may be associated with the findings. First is the question of oxygen uptake kinetics. Slower oxygen uptake kinetics at the onset of exercise and the delay to reach steady state to supply the demands of the task via aerobic means may be attributable to poor oxygen utilization of the working muscles, thus relating to neuromuscular inefficiency (Kemps et al., 2010). Poor oxygen utilization can be explained by the following mechanisms: (a) possible co-contraction of agonist and antagonist muscle groups, (b) increased neuromotor recruitment of more muscles at the same time or differing times for a given task (hyperadduction), (c) decreased blood flow through the capillaries due to increased constriction of working muscles, or (d) muscle membrane repair or damage. 
Second is the question of a somewhat counterintuitive inverse relationship between metabolic cost and perceived effort for the reading task, based on inspection of group data. Based on data from the general exercise physiology domain, the expectation was that increased metabolic cost for a task would correspond with an increased sense of intra-task effort. However, in the present study, subjects with numerically higher metabolic cost- that is, subjects in the CV group -- reported decreased task effort. Additionally, groups presenting with poor and slower oxygen uptake kinetics at task onset (including VF and VH groups) indicated increased task effort. Although seemingly paradoxical, tentative explanations are readily had. First, CV group have a greater aerobic capacity due to their aerobic training, aiding in the ability to better tolerate the increased cost of reading, thereby decreased task effort (Julius, Brach, Wert \& Van Swearingen, 2012). In the VF and VH groups illustrating poor oxygen uptake kinetics, the recruitment of anaerobic means of energy supply and the increased neuromotor recruitment at the onset of fatigue (Boucher \& Ayad, 2009, Hortobagyi et al., 2011) results in a discrepancy in the force required by the muscles to produce the task and the force perceived by the brain for completion of the task (Lambert et al., 2005; Noakes et al., 2004). Such discrepancy may have resulted in the increased sense of task effort. Second, the reading task recruited not only laryngeal muscle activity, but also respiratory and articulatory muscles. Especially the respiratory musculature is vastly larger and thus potentially more metabolically active as a whole than the laryngeal musculature. It seems entirely likely that subjects in the CV group incurred more metabolic activity overall than subjects in VF and VH groups because they recruited more respiratory "support" in speaking than subjects in the other groups, who instead may have relied more on the tiny laryngeal musculature to drive performance. Such respiratory effort, distributed 
across the relatively massive respiratory musculature, would likely induce a smaller sense of fatigue than metabolic effort directed at the larynx.

A third issue regards mechanisms in post-task oxygen consumption. Longer recovery times and greater post-task oxygen consumption point to cardiovascular recovery deficits. CV group illustrated lower post oxygen consumption compared to VF and VH groups. Such results may be influenced by aerobic training status (VH and VF had no history of cardiovascular

training). Post -task oxygen consumption (EPOC) replenishes depleted resources (including oxygen deficit incurred at the onset of task, resynthesis of ATP, replenishment of PCr, glycogen etc), removal of metabolic waste to return body to baseline levels. Aerobic training increases cardiac output and circulatory response to exercise. In other words, aerobic conditioning results in pumping more blood at a faster rate from the heart aiding quick recovery from an activity to normal (Tomlin \& Wenger, 2001). Further, aerobic training aids in fast regulation of post exercise metabolism irrespective of work rate for a given activity (Short \& Sedlock, 1997; Tomlin \& Wenger, 2001).

\subsection{SUMMARY AND CONCLUSIONS}

The present study revealed some interesting findings that may provide preliminary insight into possible metabolic mechanisms in vocal fatigue. Subjects in the VF group showed a consistent rise in oxygen uptake kinetics during a reading task, compared to subjects in VH and CV groups. Slower uptake is attributable to increased reliance on anaerobic means for task performance, which in turn creates oxygen deficit. The amount of deficit incurred is a likely source of perceived task effort. In fact, individuals with vocal fatigue reported increased vocal effort for 
both conversational and loud reading relating to their poor oxygen uptake kinetics and greater oxygen deficit. These findings point to a possible role of poor neuromuscular efficiency (possible co-contraction; increased neuromotor recruitment; decreased blood flow to muscles) underlying vocal fatigue. Additionally, the amount of oxygen deficit incurred at the onset of exercise in general influences the recovery time from the task (Mc Cardle et al., 2001, 2007). A greater number of individuals in the $\mathrm{VF}$ and $\mathrm{VH}$ groups showed an increase in post-task oxygen consumption (EPOC) during the recovery period, compared to the CV group. Excess oxygen consumed post task is used to replenish metabolic resources depleted by the task and return the body to baseline status. Increased post exercise oxygen consumption relates to poor pay back of the depleted resources. This finding suggests the role of cardiovascular recovery deficits (i.e., poor aerobic training) underlying vocal fatigue.

However, as noted, the results should be interpreted with caution, given generally similar findings for subjects in VF and VH groups. Potentially, this similarity is attributable to both recruitment methods and subject characteristics, as previously discussed. Future studies should attempt to control for these variables.

Additionally, data from the CV group point to physiologic adaptations with aerobic training and its influence on metabolic cost and cardiovascular recovery. Aerobic training enhances the phenomenon of "pay as you go" during the activity of reading. Clearly, oxygen utilization was better at the level of working muscles during the vocal task in this group possibly due to better blood flow to working muscles and adequate recruitment of muscle groups (better neuromuscular efficiency). Additionally, aerobic training appeared associated with the ability to "pay back" or replenish metabolic resources depleted with task performance, and to remove metabolic waste. This ability can be attributed to improved cardiac output as a result of aerobic 
training. Overall, data from gross metabolic cost and recovery time imply a role of aerobic training/cardiovascular conditioning and their influence on recovery from reading.

To summarize, results from this study point to (a) a possible role of neuromuscular inefficiency and cardiovascular recovery deficits as potential mechanisms underlying vocal fatigue, (b) aerobic conditioning as a means to generate energy required for task demands (aerobic training will allow for quick utilize oxygen as an energy source as opposed to anaerobic sources), and finally (c) an influence of aerobic training in amount and duration of recovery post reading- aerobically trained recover quickly compared to untrained individuals.

\subsection{LIMITATIONS AND FUTURE DIRECTIONS}

Descriptive data revealed interesting patterns of oxygen uptake and perceived effort during the reading task, and EPOC post task, across VF versus CV groups. However, in general, findings for VF versus VH groups were generally indistinct. In particular, both recruitment methods and lack of control for occupational voice use may account for this indistinction and as noted, should be addressed in future studies.

An additional limitation was that the reading task may have not been sensitive enough to capture group variations in metabolic cost and recovery time. Future protocols could increase the duration of the task to 10 minutes or longer, to increase the likelihood that all participants reach a steady state in oxygen uptake to observe patterns of uptake kinetics and recovery more fully and more realistically representative of daily talking. Future protocols could also use tasks involving greater variations of intra-task intensity, (e.g., read at conversational and loud levels within the

same task), to better parallel protocols in the exercise physiology domain, which has used 
intensity change-ups to provides insight into mechanisms of fatigue (Ratkevicius et al., 2006). Future studies should also control for cardiovascular fitness in individuals with vocal fatigue and assess its influence on gross metabolic cost during reading and recovery time.

A further issue is that recovery time between tasks in the current protocol was individual specific. In future protocols, a better idea might be to maintain a consistent inter-task duration, an approach that may provide more information on EPOC and recovery slope with time. Current data suggest that some vocally healthy individuals and those with vocal fatigue rely more on anaerobic means to supply the demands of a vocal task more than cardiovascular trained individuals. To further explore underlying mechanisms, future protocols should also incorporate assessment of lactic acid following reading tasks. In addition, measures to identify oxygen saturation (e.g., using a tissue oximeter) at the level of laryngeal and respiratory muscles might provide further insight in to the utilization of aerobic versus anaerobic means during vocal tasks.

An additional comment relates to vocal efficiency, which was variable across groups. Vocal efficiency is influenced by various factors including intensity, subglottal pressure, glottal width and contraction of vocalis and cricothyroid muscles. In order to achieve better estimates of vocal efficiency, future protocols might control for some of the noted variables (e.g., subglottic pressure or intensity) during data collection. Additionally, future studies should incorporate a group thought or shown to demonstrate especially good vocally efficiency (e.g., professional singers) to assess the influence of vocal efficiency and skill on metabolic cost.

Finally, the oxygen consumption recorded during the reading task is a global measure of the physiological energy expenditure during a task and during task recovery. In the case of reading, such cost reflects not only metabolic activity at the laryngeal level, but also at respiratory and articulatory levels and the central nervous system. Thus, future protocols could 
use measures such as infrared spectroscopy to study oxygen saturation at the level of various muscles to provide a more precise window into laryngeal, respiratory, or articulatory mechanisms in individuals with vocal fatigue.

Although the current study is undermined by some methodological limitations, the study has provided some insights into oxygen uptake and recovery kinetics and its possible role in mechanisms of vocal fatigue, laying a foundation for future research. 


\title{
APPENDIX A
}

\author{
RECRUITMENT FLYER - HEALTHY FEMALES \\ RESEARCH SUBJECTS NEEDED- HEALTHY FEMALES AGES 18-45
}

Are you interested in understanding the mechanism of your voice use? This research study is aimed to understand voice use, specifically, this research study targets the oxygen consumed during the task of talking. You will be compensated for your time.

WHO SHOULD PARTICPATE IN THIS STUDY:

- Healthy females (cardiovascularly conditioned individuals, professional singers or actors, and non-singers) in the age range of 18-45 years

- No complaints of a voice problem

- Not a current smoker

- No medication use (exception of medications that regulate menstrual cycle)

WHAT DOES THE STUDY INVOLVE:

-One screening and 1 additional session over a 1-week period (total time: $90 \mathrm{~min}$ )

-Reading in to a mask at two different loudness levels

For more information on the study, please contact Chaya Nanjundeswaran at mechanismsvf@gmail.com or call at 423-439-4036.

\begin{tabular}{|c|c|c|c|c|c|c|c|c|c|c|c|c|c|c|}
\hline 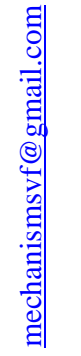 &  &  & 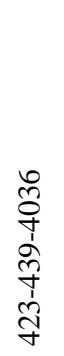 & 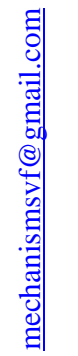 & 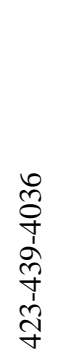 &  & 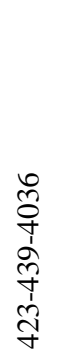 &  & 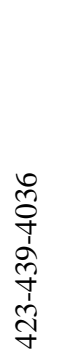 &  & 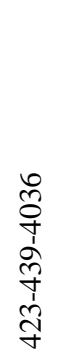 & 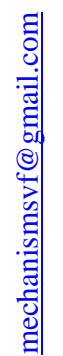 & 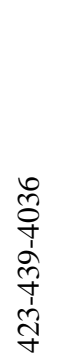 & 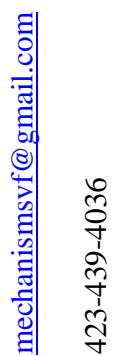 \\
\hline
\end{tabular}




\title{
APPENDIX B
}

\section{APPENDIX B-RECRUITMENT FLYER - VOICE PROBLEMS}

\author{
RESEARCH SUBJECTS NEEDED- FEMALES AGES 18-45
}

Are you interested in understanding the mechanism of your voice use? This research study is aimed to understand voice use, specifically, this research study targets the oxygen consumed during the task of talking. You will be compensated for your time.

\section{WHO SHOULD PARTICIPATE IN THE STUDY:}

- you are a female between the age of 18 and 45 years

- you have a presenting complaint of a voice problem, especially you have complaints of vocal fatigue/ tiredness of voice with voice use

- you are not a current smoker

- you are in good physical and mental health

WHAT DOES THE STUDY INVOLVE:

-One screening and 1 additional session over a 1-week period (total time: 90 min) -Reading in to a mask at two different loudness levels

\begin{tabular}{|c|c|c|c|c|c|c|c|c|c|c|c|c|c|}
\hline 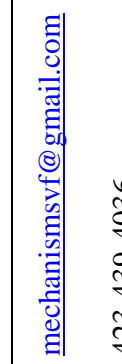 & 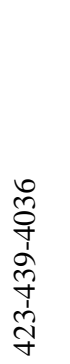 &  & 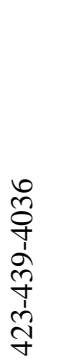 & 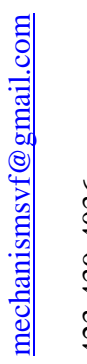 & 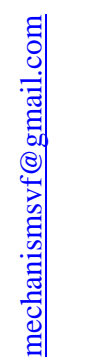 & 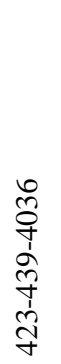 & 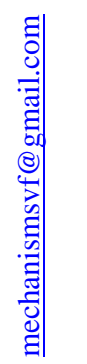 & 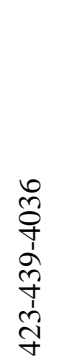 & 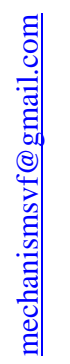 & 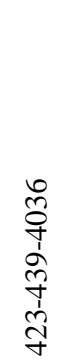 &  & 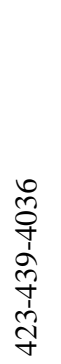 & 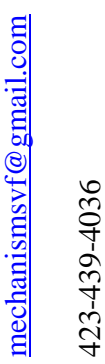 \\
\hline
\end{tabular}




\section{APPENDIX C}

\section{VOCAL FATIGUE INDEX (VFI)}

Name: $\quad$ Age/Sex:

Date: _- '_- ' -

These are some symptoms usually associated with voice problems. Circle the response that indicates how frequently you experience the same symptoms (0- never, 1 - almost never, 2sometimes, 3- almost always, 4- always).

\section{$\underline{\text { Part } 1}$}

1) I don't feel like talking after a period of voice use

$\begin{array}{lllll}0 & 1 & 2 & 3 & 4\end{array}$

2) My voice feels tired when I talk more

$\begin{array}{lllll}0 & 1 & 2 & 3 & 4\end{array}$

3) I experience increased sense of effort with talking

$\begin{array}{lllll}0 & 1 & 2 & 3 & 4\end{array}$

4) My voice gets hoarse with voice use

$\begin{array}{lllll}0 & 1 & 2 & 3 & 4\end{array}$

5) It feels like work to use my voice

$\begin{array}{lllll}0 & 1 & 2 & 3 & 4\end{array}$

6) I tend to generally limit my talking after a period of

$\begin{array}{lllll}0 & 1 & 2 & 3 & 4\end{array}$ voice use

7) I avoid social situations when I know I have to talk more

$\begin{array}{lllll}0 & 1 & 2 & 3 & 4\end{array}$

8) I feel I cannot talk to my family after a work day

$\begin{array}{lllll}0 & 1 & 2 & 3 & 4\end{array}$

9) It is effortful to produce my voice after a period of voice use

10) I find it difficult to project my voice with voice use

$\begin{array}{lllll}0 & 1 & 2 & 3 & 4\end{array}$

11) My voice feels weak after a period of voice use

$\begin{array}{lllll}0 & 1 & 2 & 3 & 4\end{array}$

\section{$\underline{\text { Part } 2}$}

12) I experience pain in the neck at the end of the day with voice use 
13) I experience throat pain at the end of the day with voice use

14) My voice feels sore when I talk more

$\begin{array}{lllll}0 & 1 & 2 & 3 & 4\end{array}$

15) My throat aches with voice use

$\begin{array}{lllll}0 & 1 & 2 & 3 & 4\end{array}$

16) I experience discomfort in my neck with voice use

$\begin{array}{lllll}0 & 1 & 2 & 3 & 4\end{array}$

\section{$\underline{\text { Part } 3}$}

17) My voice feels better after I have rested

18) The effort to produce my voice decreases with rest

19) The hoarseness of my voice gets better with rest $\begin{array}{lllll}0 & 1 & 2 & 3 & 4\end{array}$

$\begin{array}{lllll}0 & 1 & 2 & 3 & 4\end{array}$

$\begin{array}{lllll}0 & 1 & 2 & 3 & 4\end{array}$ 


\section{APPENDIX D}

\section{READING PASSAGES}

When you look at mountains, ranges and hills, they always seem permanent and unchanging. Yet for millions of years, Earth's surface has gradually changed or continues to evolve. Darwin suggested that plant and animal life evolved and he explained those changes. Early in his life he was fascinated with the plant and animal kingdoms. He observed the world collecting fossils and rocks. In 1830, he joined an important exploratory voyage along the beautiful coasts of South America. He noted that the characteristics of animals differed from island to island. By studying the characteristics of turtles, he could tell exactly which island it came from. He studied fourteen varieties of finches. His explanation of the changes is often called the survival of the fittest. To understand this concept, think about the plants and animals on the earth's surface. Every minute, thousands of organisms come into existence. But there is a problem with this theory, as there is only a certain amount of food, nourishment and oxygen to go around. It is not possible for organisms to survive on the limited resources. Darwin spent his life explaining these factors. His famous theory is accepted.

Columbia, the first reusable spacecraft was launched in April 1981. This space shuttle also acts like a rocket, but it lands like a regular airplane. What makes this spacecraft special is that, it was built so that it could be used for at least one hundred times. The shuttle consists of an 
orbiter, a tank for fuel and two rocket boosters. The orbiter carries seven astronauts and sixty five thousand pounds of equipment. The shuttle is able to carry people and other items between earth and space. Satellites can be placed into earth's orbit, picked up and returned to earth for repairs or can be replaced by new satellite. A manned space lab is being currently planned between 1999 and 2002. This lab will allow scientists to conduct experiments in physical sciences. The lab will remain attached to a station throughout the entire mission. Military space stations would act mainly as communication centers and can be used to train astronauts from the United States. Future plans for the shuttle includes the building of a station somewhere in outer space. The shuttle would go between the main space and earth carrying supplies such as fuel to be used to launch vehicles into space.

In the 1790 's, Eli Whitney traveled to the south to study law. While visiting a cotton plantation, he was interested in growing cotton. He saw that the production of cotton was slow. It took all day to pick seeds from one pound of cotton as the cotton seeds had to be removed by hand. To solve this problem, Whitney invented a powerful machine called the Cotton Gin. The machine had a roller with wire teeth. The teeth pulled the pieces of cotton through the opening which was too small for the seeds to pass through. This simple machine changed the cotton business. In less than one year after the invention, the sale of the US cotton crop grew. Five to eight million pounds of cotton was grown every year. Within six years, thirty seven million pounds of cotton crop were produced. Whitney was not careful in guarding his work. He could not make the gins to meet the demand. Planters eager to increase their production copied his ideas and made their own machines. By reducing the cost, the cotton gin made the growing of cotton a good business. Because cotton was not grown in Europe, it was a very important product 
that Americans could sell to other nations. Cotton was imported from United States and the US made huge profits on exports.

Eddie was a carpet fitter, and he hated it. For ten years he had spent his days sitting, squatting, kneeling or crawling on floors, in houses, offices, shops, factories and restaurants. Ten years of his life, cutting and fitting carpets for other people to walk on, without even seeing them. When his work was done, no one ever appreciated it. No- one ever said, "Oh, that's a beautiful job, the carpet fits so neatly." They just walked all over it. Eddie was sick of it. He was especially sick of it on this hot, humid day in August, as he worked to put the finishing touches to today's job. He was just cutting and fixing the last edge on a huge red carpet, which he had fitted in the living room of Mrs. Smith's house. Mrs. Smith, who changed her carpets every year and always, bought the best. Mrs. Smith, who had never even given him a cup of tea all day, and who made him go outside when he wanted to smoke. It was four o'clock and he had nearly finished. At least he would be able to get home early today. He began to day-dream about the weekend, about the Saturday football game he always played for the local team, where he was known as "Ed the Head" for his skill in heading goals from corner kicks.

Today we receive different types of vaccines. These vaccinations keep us all healthy. The principle behind each vaccination is the same. Small doses of germs are injected into our bodies causing the blood system to produce antibodies to fight the germs and prevent us from getting the disease. Edward Jenner was a pioneer in this field. Many years ago, a deadly disease called smallpox swept across many countries. The symptoms included chills and fever. Later, pus filled red spots covered the body. Those lucky enough to survive were often blind, handicapped or badly disfigured. Approximately, sixty million people died between 1700 and 1800 . At the same time, a much milder disease cowpox was sweeping the rural areas. It was common among people 
who milked cows. Cowpox was transferred from the udder of an infected cow to the hands of the milker. It was a superstition that anyone who was infected with cowpox could not get smallpox. Jenner changed this idea from superstition to scientific fact using his research. For twenty years, he studied the records and carefully documented the cases. He concluded that milkmaids did not get smallpox because; mild cases of cowpox had provided them with the immunity. He believed others could become immune by infecting them with new cowpox germs.

Heart is an important part of the body and pumps blood to all parts of the body. This blood delivers needed oxygen to the brain. One of the most common causes of death in elders is heart failure. Heart can fail to work for many reasons. In some cases, people are born with hearts which are usually damaged during birth. People can also damage their hearts if they do not eat correctly or exercise enough. Smoking can also lead to heart problems. A number of ways of helping to improve the heart has been recently developed. Some heart problems are solved simply by the regular use of correct medications. More serious problems may need surgery. Often the arteries of the heart get clogged and surgeons replace sections of arteries, so that the blood is able to flow better. Small machines called pace makers are placed next to the heart to help it beat regularly. Transplants, either human or artificial have worked for only a short period of time. Most patients do not survive because their bodies reject a transplanted organ. However, the patient's chances are good the first year of living with a transplant. With continuing improvements, people with failed hearts may soon be able to live out lives with the help of a new heart transplant.

The radio was developed with the help of many inventors from different countries. Scientists discovered that electrical waves and light waves should be closely related. In 1890, an US inventor developed the first wireless telegraph. An American citizen was the first to 
broadcast live voice by radio waves. By 1920, the first radio station was set up in the United States. Radio was an important improvement in the history of communication. For the first time, people around the world were brought closer together by listening to news and events from other countries. Comedy, drama, and sports shows created many famous sportsmen and movie stars. Radios continue to be popular mainly because they are the best means for broadcasting live and interesting music. Ships and planes depend on radios for navigation and strategic details. The military uses radio for communication and to track the paths of guided missiles and rockets. Scientists can talk to astronauts in space and learn more about other planets and stars. Meteorologists even learn about the atmosphere from the weather balloons. Recently, satellites have been used to transmit radio waves directly to radio units at home or in cars.

When the sunlight strikes raindrops in the air, they act like a prism and form a rainbow. The rainbow is a division of white light into many beautiful colors. These take the shape of a long round arch, with its path high above, and its two ends apparently beyond the horizon. There is, according to legend, a boiling pot of gold at one end. People look, but no one ever finds it. When a man looks for something beyond his reach, his friends say he is looking for the pot of gold at the end of the rainbow. Throughout the centuries men have explained the rainbow in various ways. Some have accepted it as a miracle without physical explanation. To the Hebrews it was a token that there would be no more universal floods. The Greeks used to imagine that it was a sign from the gods to foretell war or heavy rain. The Norseman considered the e rainbow as a bridge over which gods passed from earth to their home in the sky. Other men have tried to explain the phenomenon physically. Aristotle thought that the rainbow was caused by reflection of the sun's rays by the rain. Since then physicists have found that it is not reflection, but refraction by the raindrops which causes the rainbow. Many complicated ideas about the rainbow 
have been formed. The difference in the rainbow depends considerably upon the size of the water drops, and the width of the colored band increases as the size of the drops increases. The actual primary rainbow observed is said to be the effect of superposition of a number of bows. If the red of the second bow falls upon the green of the first, the result is to give a bow with an abnormally wide yellow band, since red and green lights when mixed form yellow. This is a very common type of bow, one showing mainly red and yellow, with little or no green or blue.

As early explorers sailed across unknown seas, they imagined that all kinds of mysterious things lived beneath the surface of the ocean. By the 1800's most of the world's oceans had been mapped, but it was not until the mid-twentieth century that people began exploring the seas. Cousteau is a pioneer in the field of oceanography. Oceanography is the study of the ocean. As an explorer, researcher, and inventor, he is credited for replacing the mystery of the seas with knowledge. Cousteau is responsible for several inventions which helped make undersea exploration possible. One invention, the aqua lung, has permitted divers to carry oxygen to depths of 300 feet. Another Cousteau invention, the diving saucer, is a small submarine used to study and retrieve objects on the ocean floor. In 1951, Cousteau set sail on his own research ship, the Calypso, exploring shipwrecks. Cousteau found a sunken Roman trading ship that was 2,000 years old, overloaded with wine and pottery. Later, Cousteau turned his attention from sea wrecks to sea exploration. Since the invention of the underwater movie camera, Cousteau has recorded his discoveries about sea life on film.

At the beginning of human history, people were left in darkness when the sunset. Then came the discovery of fire. Centuries later candles, then oil and gas lights were developed. But until the late 1800's these were the only sources of light besides the sun. All of them were dangerous, smoky, used open flames, and produced very poor lighting. Since the discovery of 
electricity, many people had been experimenting with ideas for electric lighting. It took Thomas Edison, an especially curious and persistent inventor, to find a way to use electricity. In 1879, Edison found the perfect filament, or wire, to carry enough electric current to produce the first practical light-bulb. The wire was made of carbonized thread - ordinary cotton thread burned to an ash. He knew his invention would replace the gas lamps that were being widely used. He quickly developed a power station to produce electricity so that whole streets could have electric lights. Just five years after his invention, Edison had 500 customers in New York City using electric lights. Electric lighting had many advantages over gas lamps.

Over 1,000 years ago, there lived in Europe, a fearsome band of warriors known as Norseman, or Vikings. For centuries, these Vikings terrorized Europe, murdering and stealing wherever they landed their boats. Due to a rapidly growing population and a shortage of good farmland, many of these adventurous people left in long open boats in search of new places to settle.

One of the places they explored and settled for a while was Iceland. A Viking by the name of Eric the Red was sent away from Iceland because he committed a murder. Taking a crew of men with him, he headed for a land to the west that he heard about. He named the island he found "Greenland," not a very appropriate name for a frozen land of ice and snow. Many years later, Eric's son Leif set sail for a land that had been seen by a Norseman whose ship had blown off course. Leif Ericson named this land "Vinland" because of the vines and grapes he found growing there. No one is sure where Vinland was located. Some historians believe it was Newfoundland.

An early science fiction story tells about men from Mars, invading the Earth. Their weapon was a mysterious "sword of heat," from which flickered " a ghost of a beam of light.” It 
dropped men in their tracks and made lead run like water. It turned anything that would burn into masses of flame. Today that "sword of light" is a reality in the laser; a marvelous new device that shoots out a narrow beam of the most intense light ever known. Imagine light that burns billions of times more brightly that the light at the surface of the sun! The laser can indeed make lead run like water. Used at close range, it can turn anything on earth into vapor. There is already tremendous excitement over the many different uses the laser may serve. Some experts say that laser industry will be a billion dollar industry in a few years in the United States alone. The excitement began in the late 50's, when two researchers published a description of how a laser might be made. In the early 60's, a man made ruby was used to bring forth a flash of brilliant red radiation. It was the world's first laser beam. The light fantastic was born.

You wish to know all about my grandfather. Well, he is nearly 93 years old; he dresses himself in an ancient black frock coat, usually minus several buttons; yet he still thinks as swiftly as ever. A long, flowing beard clings to his chin, giving those who observe him a pronounced feeling of the utmost respect. When he speaks, his voice is just a bit cracked and quivers a trifle. Twice each day he plays skillfully and with zest upon our small organ. Except in the winter when the ooze or snow or ice prevents, he slowly takes a short walk in the open air each day. We have often urged him to walk more and smoke less, but he always answers, "Banana Oil!" Grandfather likes to be modern in his language.

Neil Armstrong's fascination with flying dates back to his childhood, when he constructed hundreds of model planes. As a teenager, he saved his money to pay for flying lessons. After college, Armstrong worked as a test pilot. Armstrong was among the second group which formed NASA's astronaut training program. In 1966, after years of physical and academic preparation, Armstrong was appointed commander of the Gemini 8 space mission. The goal was 
to perform the first space docking, connecting two spacecraft while in orbit. After the docking, the Gemini capsule began spinning out of control. Armstrong skillfully guided Gemini 8 to an emergency splashdown. In 1969, Armstrong joined astronauts Edwin Aldrin, Jr. and Michael Collins as commander of Apollo 11, whose mission was to land humans on the moon. The dream became a reality on July 20, 1969, when Armstrong lowered the Eagle, the lunar landing module, to the moon's surface. Collins orbited the moon in the command module Columbia, while Armstrong and Aldrin explored the moon's surface. Their space suits contained supplies of air, water, and electricity, making the astronauts self-sufficient in moon's hostile environment. Each suit had a refrigeration unit, because the temperature on the moon varies more than 290 Celsius!

As early explorers sailed across unknown seas, they imagined that all kinds of mysterious things lived beneath the surface of the ocean. By the 1800's most of the world's oceans had been mapped, but it was not until the mid-twentieth century that people began exploring the seas. Smith is a pioneer in the field of oceanography. Oceanography is the study of the ocean. As an explorer, researcher, and inventor, he is credited for replacing the mystery of the seas with knowledge. Smith is responsible for several inventions which helped make undersea exploration possible. One invention, the aqua lung, has permitted divers to carry oxygen to depths of 300 feet. Another Cousteau invention, the diving saucer, is a small submarine used to study and retrieve objects on the ocean floor. In 1951, Smith set sail on his own research ship, the Calypso, exploring shipwrecks. Smith found a sunken Roman trading ship that was 2,000 years old, overloaded with wine and pottery. Later, Smith turned his attention from sea wrecks to sea exploration. Since the invention of the underwater movie camera, Smith has recorded his discoveries about sea life on film. 
In the 1700's nearly every home had a spinning wheel. Every day women and children spent hours spinning raw cotton or wool into yarn, which was gathered onto a spindle. These threads of yarn could then be used for making clothes and other household items. In 1764, in the home of Jenny and James Hargreave, an accident happened which led to an important invention. While being used, the spinning wheel fell over. No one was hurt, but James Hargreave noticed that more spindles could be added by changing the direction of the spindles. Quickly he designed a spinning wheel that could be used by one person yet could spin yarn onto eight different spindles. He named it the Spinning Jenny after his wife. The reaction to Hargreave's invention was interesting. Some people were afraid that this new invention would put those who made their living from spinning yarn out of work. Angry crowds attacked his house and destroyed his new spinning machines. The Hargreave's had to move to a new town. Soon people began to realize that the Spinning Jenny could increase production and add jobs. Spinning machines were developed to turn out as many as a hundred spindles of yarn. The Spinning Jenny became the first step in making materials and clothes in factories instead of homes.

Medical care has improved rapidly over the last 100 years. One of the most important inventions has been the X-ray machine invented by a German scientist in 1895. Rays that cannot be seen flow from a special bulb, enter the body, and leave a picture on a photographic plate that shows what things look like inside. The scientist called these rays $\mathrm{X}$ because he didn't really understand where they came from. He discovered them by accident. With the X-ray machine doctors can see exactly where a bullet is located, where a bone is broken, or the location of a cavity in a tooth. Through the use of X-rays, doctors can discover and treat many medical problems. In addition to their use by doctors, X-rays can be very helpful in industry. X-rays can be used to spot mistakes such as cracks in products which often we are not able to be seen with 
just the eyes. Doctors using the first X-ray machines did not realize they could harm the body. When used too much, the X-rays begin to destroy cells in the body and can cause cancer to grow. At first X-rays were given nearly every time a person was examined by their doctor. Now they are only used when doctors believe that something is wrong.

Printing from movable type was probably the most important invention the 1400s. Before books became widely available the only way knowledge was passed along was through teaching a small number of scholars and priests or writing information in handwritten letters. With limited transportation, people didn’t travel much and knew very little about other people and places. Once books were widely available people learned history, culture, literature, and many other things. While books had been around for centuries they took months or even years to handprint. Books were so expensive that only the very wealthy could buy them. Then, in 1440, a German inventor developed movable metal type to be used with a printing press, which transferred the type to paper. Books could now be printed with greater speed and with less error. Information and ideas could be shared with more people and at a faster rate since printers could offer books at lower prices. Before there were a lot of printed materials, the common people had few reasons to learn how to read. Once these materials became available, people became interested in studying, learning and attending school.

Every second, one hundred square meters of the world's rainforest is destroyed. That's equivalent to two football fields. An area the size of New York City is lost every day. In a year that adds up to 31 million hectares, that is more than the land area of Poland. This alarming rate of destruction has serious consequences for the environment. Scientists estimate that 137 species of plant, insect or animal become extinct every day due to logging. In British Columbia, where, since 1990, thirteen rainforest valleys have been clearcut, 142 species of salmon have already 
become extinct, and the habitats of grizzly bears, wolves and many other creatures are threatened. Logging, however, provides jobs, profits, taxes for the government and cheap products of all kinds for consumers, so the government is reluctant to restrict or control it. Much of Canada's forestry production goes towards making pulp and paper. According to the Canadian Pulp and Paper Association, Canada supplies 34\% of the world's wood pulp and 49\% of its newsprint paper. If these paper products could be produced in some other way, Canadian forests could be preserved. Recently, a possible alternative way of producing paper has been suggested by agriculturalists and environmentalists. 
APPENDIX E

BORG RATINGS OF PERCEIVED EXERTION 


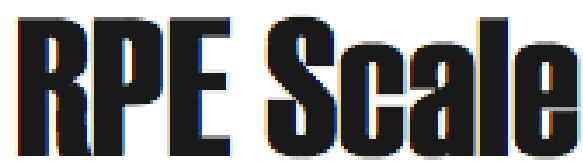

rating of perealved axertion



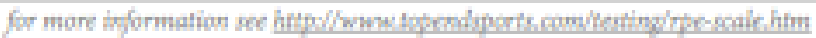




\section{BIBLIOGRAPHY}

Adams, G. R., Fisher, M. J., \& Meyer, R. A. (1991). Hypercapnic acidosis and increased H2PO4- concentration do not decrease force in cat skeletal muscle. American Journal of Physiology, 260(4 Pt 1), C805-812

Allen, D. G., Lamb, G. D., \& Westerblad, H. (2008). Skeletal muscle fatigue: cellular mechanisms. Physiol Rev, 88(1), 287-332.

Amann, M., \& Calbet, J. A. (2008). Convective oxygen transport and fatigue. J Appl Physiol, 104(3), 861-870.

Amann, M., \& Dempsey, J. A. (2008). The concept of peripheral locomotor muscle fatigue as a regulated variable. $J$ Physiol.

Anderson, P., \&, \& Hendriksson, J. (1977). Training induced changes in the subgroups of human type II skeletal muscle fibers. Acta Physiol Scand, 99, 123-125.

Asmussen, E. (1979). Muscle fatigue. Med Sci Sports, 11(4), 313-321.

Astrand, P. O., Rodahl, K., Dahl, H. A., \&, \& Stromme, S. B. (2003). Textbook of Work Physiology: Physiological Bases of Exercise (4th ed.): Human Kinetics.

Bahr, R., \& Sejersted, O. M. (1991). Effect of intensity of exercise on excess postexercise O2 consumption. Metabolism, 40(8), 836-841.

Bangsbo, J., \& Juel, C. (2006). Lactic acid accumulation is an advantage/disadvantage during muscle activity. Journal of Applied Physiology, 100, 1410-1414.

Berke, G.S., Hanson, D.G., Gerratt, B.R., \& Trapp, T.K. (1990). The effect of air flow and medial adductory compression on vocal efficiency and glottal vibration. Otolaryngology and Head and Neck Surgery, 102, 212-218.

Bigland-Ritchie, B. (1981). EMG/force relations and fatigue of human voluntary contractions. Exercise and Sport Sciences Reviews, 9, 75-117.

Bigland-Ritchie, B., Cafarelli, E., \& Vollestad, N. K. (1986). Fatigue of submaximal static contractions. Acta Physiol Scand Suppl, 556, 137-148.

Bigland-Ritchie, B., Jones, D. A., Hosking, G. P., \& Edwards, R. H. (1978). Central and peripheral fatigue in sustained maximum voluntary contractions of human quadriceps muscle. Clin Sci Mol Med, 54(6), 609-614.

Borg, G. A. (1982). Psychophysical bases of perceived exertion. Medicine and Science in Sports and Exercise, 14(5), 377-381.

Boucher, V. J., Ahmarani, C., \& Ayad, T. (2006). Physiologic features of vocal fatigue: electromyographic spectral-compression in laryngeal muscles. Laryngoscope, 116(6), 959-965.

Boucher, V. J., \& Ayad, T. (2009). Physiological Attributes of Vocal Fatigue and Their Acoustic Effects: A Synthesis of Findings for a Criterion-Based Prevention of Acquired Voice Disorders. $J$ Voice. 
Brehm, M., Knol, D.L., \& Harlaar, J. (2008). Methodological considerations for improving the reproducibility of walking efficiency outcomes in clinical gait studies. Gait \& Posture, 27, 196-201.

Brooks, G. A., \& Donovan, C. M. (1983). Effect of endurance training on glucose kinetics during exercise. Am J Physiol, 244(5), E505-512.

Brooks, G. A., Fahey, T. D., \& , \& Baldwin, K. M. (2005). Exercise Physiology: Human Bioenergetics and its Applications (4th ed.): McGraw Hill.

Brooks, G. A., Hittelman, K. J., Faulkner, J. A., \& Beyer, R. E. (1971). Temperature, skeletal muscle mitochondrial functions, and oxygen debt. Am J Physiol, 220(4), 1053-1059.

Buekers, R. (1998). Are voice endurance tests able to assess vocal fatigue? Clinical Otolaryngology and Allied Sciences, 23(6), 533-538.

Chang, A., \& Karnell, M. P. (2004). Perceived phonatory effort and phonation threshold pressure across a prolonged voice loading task: a study of vocal fatigue. $J$ Voice, 18(4), 454-466.

Chilibeck, P.D., Paterson, D.H., Petrella, R. J. \& Cunningham, D.A. (1996). The influence of age and cardiorespiratory fitness on kinetics of oxygen uptake. Canadian Journal of Applied Physiology, 21 (3), 185-196.

Cohen-Solal, A., Laperche, T., Morvan, D., Geneves, M., Caviezel, B., \& Gourgon, R. (1995). Prolonged kinetics of recovery of oxygen consumption after maximal graded exercise in patients with chronic heart failure. Analysis with gas exchange measurements and NMR spectroscopy. Circulation, 91 (12), 2924-2932.

Colliander, E. B., Dudley, G. A., \& Tesch, P. A. (1988). Skeletal muscle fiber type composition and performance during repeated bouts of maximal, concentric contractions. Eur J Appl Physiol Occup Physiol, 58(1-2), 81-86.

Colton, R. H., Casper, J. K., \& , \& Leonard, R. (2006). Understanding voice problems: A physiological perspective for diagnosis and treatment (3rd ed.). Baltimore, MD: Lippincott Williams \& WIlkins.

Davis, J. M., \& Bailey, S. P. (1997). Possible mechanisms of central nervous system fatigue during exercise. Med Sci Sports Exerc, 29(1), 45-57.

Dahlstedt, A. J., \& Westerblad, H. (2001). Inhibition of creatine kinase reduces the rate of fatigue-induced decrease in tetanic $[\mathrm{Ca}(2+)](\mathrm{i})$ in mouse skeletal muscle. Journal of Physiology, 533(Pt 3), 639-649.

Degens, H., Horneros, J. M. S., \&, \& Hopman, M. T. E. (2006). Acute hypoxia limits endurance but does not affect muscle contractile properties. Muscle Nerve, 33, 532-537.

Edwards, R. H. T. (1981). Human muscle function and fatigue. In R. P. a. J. Whelan (Ed.), Human Muscle Fatigue: Physiological Mechanisms (pp. 1-18). London: Pitman Medical.

Ekblom, B., \& Hermansen, L. (1968). Cardiac output in athletes. J Appl Physiol, 25(5), 619-625.

Enoka, R. M., \& Stuart, D. G. (1992). Neurobiology of muscle fatigue. J Appl Physiol, 72(5), 1631-1648.

Fitts, R. H. (1994). Cellular mechanisms of muscle fatigue. Physiol Rev, 74(1), 49-94.

Gaesser, G. A., \& Brooks, G. A. (1984). Metabolic bases of excess post-exercise oxygen consumption: a review. Med Sci Sports Exerc, 16(1), 29-43.

Gandevia, S. C. (2001). Spinal and supraspinal factors in human muscle fatigue. Physiol Rev, 81(4), 1725-1789.

Gandevia, S. C., Enoka, R. M., McComas, A. J., Stuart, D. G., \& Thomas, C. K. (1995). Neurobiology of muscle fatigue. Advances and issues. Adv Exp Med Biol, 384, 515-525. 
Gelfer, M.P., \& Andrews, M.L. (1991). Effects of prolonged loud reading on selected measures of vocal function in trained and untrained singers. Journal of Voice, 5, 158-167.

Gonzalez-Alonso, J., \& Calbet, J. A. (2003). Reductions in systemic and skeletal muscle blood flow and oxygen delivery limit maximal aerobic capacity in humans. Circulation, 107(6), 824-830.

Gotaas, C., \& Starr, C. D. (1993). Vocal fatigue among teachers. Folia Phoniatr (Basel), 45(3), 120-129.

Gillespie, A.I., Gartner-Schmidt, J., Rubinstein, E.N., \& Verdolini-Abbott (2013). Aerodynamic profiles of females with muscle tension dysphonia/aphonia. Journal of Speech, Language, and Hearing Research, 56 (2), 481-488.

Grillo, E.U., \& Verdolini, K. (2008). Evidence for distinguishing pressed, normal, resonant, and breathy voice qualities by laryngeal resistance and vocal efficiency in vocally trained subjects. Journal of Voice, 22 (5), 546-552.

Hagberg, J.M., Hickson, R.C., Ehsani, A.A, \& Holloszy, J.O. (1980). Faster adjustment to and recovery from submaximal exercise in the trained state. Journal of Applied Physiology, 48 (2), 218-224.

Hill, A. V., Long, C. N. H., \& , \& Lupton, H. (1924). Muscular exercise, lactic acid, and the supply and utilization of oxygen. The oxygen debt at the end of exercise. . Paper presented at the Proceedings of the Royal Society of London. Series B, Containing Papers of a Biological Character.

Hirano, M. (1974). Morphological structure of the vocal cord as a vibrator and its variations. Folia Phoniatrica, 26, 89-94.

Hogan, M.C., Kohin, S., Stary, C.M., \& Hepple, R.T. (1999). Rapid force recovery in contracting skeletal muscle after brief ischemia is dependent on $\mathrm{O}_{2}$ availability. Journal of Applied Physiology, 87, 2225-2229.

Holloszy, J. O., \& Coyle, E. F. (1984). Adaptations of skeletal muscle to endurance exercise and their metabolic consequences. J Appl Physiol, 56(4), 831-838.

Holmberg, E. B., Hillman, R. E., \& Perkell, J. S. (1988). Glottal airflow and transglottal air pressure measurements for male and female speakers in soft, normal, and loud voice. $J$ Acoust Soc Am, 84(2), 511-529.

Hortibagyi, T., Finch, A., Solnik, S., Rider, P., \& De Vita, P. (2011). Association between muscle activation and metabolic cost of talking in young and old adults. Journal of Gerontology, 66 (5), 541-547.

Hunter, E. J., \& Titze, I. R. (2009). Quantifying vocal fatigue recovery: dynamic vocal recovery trajectories after a vocal loading exercise. Ann Otol Rhinol Laryngol, 118(6), 449-460.

Jacks, D., Moore, J. B., Topp, R., \& Bibeau, W. S. (2008). Prediction of VO2 Peak Using a Submaximal Bench Step Test in Children: 2268: Board \#48 May 30 3:30 PM- 5:00 PM. Medicine \& Science in Sports \& Exercise, 40(5), S418 410.1249/1201.mss.0000322780.0000329703.0000322713.

Jacobson, B.H., Johnson, A., Grywalski, C., Silbergleit, A.K., Jacobson, G.P. Benninger, M.S., \& Newman, C.W. (1997). The voice handicapp index (VHI): development and validation. American Journal of Speech-Lnaguage Pathology, 6 (3), 66-70.

Jiang, J., Stern, J., Chen, H. J., \& Solomon, N. P. (2004). Vocal efficiency measurements in subjects with vocal polyps and nodules: a preliminary report. Ann Otol Rhinol Laryngol, 113(4), 277-282. 
Jones, A.M., \& Carter, H. (2000). The effect of endurance training on parameters of aerobic fitness. Sports Medicine, 29 (6), 373-386.

Julius, L.M., Brach, J.S., Wert. D.M., \& Van Swearingen, J.M. (2012). Perceived effort of walking: Relationship with gait, physical function, fear of falling, and confidence in walking in older adults with mobility limitations. Physical Therapy, 92, 1268-1277.

Kasch, F. W., \&, \& Boyer, J. L. (1968). Adult Fitness Principles and Practice. San Diego: San Diego State College.

Kasch, F. W., Phillips, W. H., Ross, W. D., Carter, J. E., \& Boyer, J. L. (1966). A comparison of maximal oxygen uptake by treadmill and step-test procedures. J Appl Physiol, 21(4), 1387-1388.

Kemps, H.M., Schep, G., Zonderland, M.L., Thijssen, E.J., De Vries, W.R., Wessels, B. et al. (2010). Are oxygen uptake kinetics in chronic heart failure limited by oxygen delivery or oxygen utilization? International Journal of Cardiology, 142, 138-144.

Kent-Braun, J. A. (1997). Noninvasive measures of central and peripheral activation in human muscle fatigue. Muscle Nerve Suppl, 5, S98-101.

Kitch, J. A., \& Oates, J. (1994). The perceptual features of vocal fatigue as self-reported by a group of actors and singers. $J$ Voice, 8(3), 207-214.

Koike, Y., \& Hirano, M., (1973). Glottal-area time function and subglottal-pressure variation. Journal of the Acoustical Society of America, 54, 1618-1627.

Kostyk, B. E., \& Putnam Rochet, A. (1998). Laryngeal airway resistance in teachers with vocal fatigue: a preliminary study. $J$ Voice, 12(3), 287-299.

Koufman, J. A., \& Blalock, P. D. (1988). Vocal fatigue and dysphonia in the professional voice user: Bogart-Bacall syndrome. Laryngoscope, 98(5), 493-498.

Lambert, E. V., St Clair Gibson, A., \& Noakes, T. D. (2005). Complex systems model of fatigue: integrative homoeostatic control of peripheral physiological systems during exercise in humans. Br J Sports Med, 39(1), 52-62.

Lamb, G. D., \&, \& Stephenson, D. G. (2006). Lactic acid accumulation is an advantage/disadvantage during muscle activity. Journal of Applied Physiology, 100, 1410-1414.

Lannergren, J., \& Westerblad, H. (1991). Force decline due to fatigue and intracellular acidification in isolated fibres from mouse skeletal muscle. Journal of Physiology, 434, 307-322.

McCabe, D. J., \& Titze, I. R. (2002). Chant therapy for treating vocal fatigue among public school teachers: A preliminary study. American Journal of Speech-Language Pathology, 11, 356-369.

McCardle, W. D., Katch, F. I., \& , \& Katch, V. L. (2001). Exercise Physiology: Energy, Nutrition, and Human Performance: Lippincott Williams \& Wilkins.

McCardle, W. D., Katch, F. I., \& , \& Katch, V. L. (2007). Exercise Physiology: Energy, Nutrition, and Human Performance: Lippincott Williams \& Wilkins.

Morton, V., \& Watson, D. R. (1998). The teaching vocie: problems and perceptions. Logopedics, Phoniatrics, Vocology, 23, 133-139.

Myburgh, K. H. (2004). Can any metabolites partially alleviate fatigue manifestations at the cross-bridge? Med Sci Sports Exerc, 36(1), 20-27.

Myers, J., \& Madhavan, R. (2001). Exercise testing with gas exchange analysis. Cardiol Clin, 19(3), 433-445. 
Nanjundeswaran, C., Jacobson, B., Gartner-Schmidt, J., Rosen, C., \& Verdolini-Abbott (Under review). Vocal Fatigue Index: Development and Validation.

Noakes, T. D., St Clair Gibson, A., \& Lambert, E. V. (2004). From catastrophe to complexity: a novel model of integrative central neural regulation of effort and fatigue during exercise in humans. Br J Sports Med, 38(4), 511-514.

Noakes, T. D., St Clair Gibson, A., \& Lambert, E. V. (2005). From catastrophe to complexity: a novel model of integrative central neural regulation of effort and fatigue during exercise in humans: summary and conclusions. Br J Sports Med, 39(2), 120-124.

Orlikoff, R.F., \& Baken, R.J. (1990). Consideration of the relationship between the fundamental frequency of phonation and vocal jitter. Folia Phoniatrica (Basel), 42, 31-40.

Orlikoff, R.F., \& Kahane, J.C. (1991). Influence of mean sound pressure level on jitter and shimmer measures. Journal of Voice, 5, 113-119.

Paul, L., \& Wood, L. (2002). Skeletal muscle fatigue. Physical Therapy Reviews, 7, 123-132.

Poole, D. C., Barstow, T. J., Gaesser, G. A., Willis, W. T., \& Whipp, B. J. (1994). VO2 slow component: physiological and functional significance. Med Sci Sports Exerc, 26(11), 1354-1358.

Poole, D. C., Barstow, T. J., McDonough, P., \& Jones, A. M. (2008). Control of oxygen uptake during exercise. Med Sci Sports Exerc, 40(3), 462-474.

Poole, D. C., Schaffartzik, W., Knight, D. R., Derion, T., Kennedy, B., Guy, H. J., et al. (1991). Contribution of exercising legs to the slow component of oxygen uptake kinetics in humans. J Appl Physiol, 71(4), 1245-1260.

Ratkevicius, A., Stasiulis, A., Dubininkaite, L., \&, \& Skurvydas, A. (2006). Muscle fatigue increases metabolic costs of ergometer cycling without changing VO2 slow component. Journal of Sports Science and Medicine, 5, 440-448.

Reybrouck, T., Vangesselen, S., Mertens, L., \& Gewillig, M. (2007). Efficiency of oxygen cost during exercise in patients with symptoms of fatigue during physical activities. Acta Paediatrics, 96(9), 1311-1314.

Rousseau, B., Hirano, S., Chan, R. W., Welham, N. V., Thibeault, S. L., Ford, C. N., et al. (2004). Characterization of chronic vocal fold scarring in a rabbit model. Journal of Voice, 18(1), 116-124.

Russell, B.A., Cerny, F.J., \& Stathopoulos, E.T. (1998). Effects of varied vocal intensity on ventilation and energy expenditure in women and men. Journal of Speech, Language, and Hearing Research, 41, 239-248.

Russell, A., Oates, J., \& Greenwood, K. (1998). Prevalence of voice problems in teachers. Journal of Voice, 12(4), 467-479.

Sahlin, K. (1992). Metabolic factors in fatigue. Sports Med, 13(2), 99-107.

Saltin, B., \& Rowell, L. B. (1980). Functional adaptations to physical activity and inactivity. Fed Proc, 39(5), 1506-1513.

Sapir, S., Mathers-Schmidt, B., \& Larson, G.W. (1996). Singers' and non-singers' vocal health, vocal behaviours, and attitudes towards voice and singing: indirect findings from a questionnaire. International Journal of Language and Communication Disorders, 31 (2), 193-209.

Scott, C. (2005). Misconceptions about aerobic and anaerobic energy expenditure. Journal of the International Society of Sports Nutrition, 2(2), 32-37. 
Scherer, R. C., Titze, I. R., Raphael, B. N., Wood, R. P., Ramig, L. A., \& , \& Blager, R. F. (1991). Vocal fatigue in a trained and an untrained voice user. San Diego, CA: Plural Publishing.

Schutte, H.K. (1980). The efficiency of voice production (kemper, Gronningen)

Scrutinio, D., Passantino, A., Lagioia, R., Napoli, F., Ricci, A., \& Rizzon, P (1998). Percent achieved of predicted peak exercise oxygen uptake and kinetics of recovery of oxygen uptake after exercise for risk stratification in chronic heart failure. International Journal of Cardiology, 64, 117-124.

Short, K.R., \& Sedlock, D.A. (1997). Excess postexercise oxygen consumption and recovery rate in trained and untrained subjects. Journal of Applied Physiology, 83 (1), 153-159.

Sims, G. (1987a). Explorers. Cypress, CA: Creative Teaching Press.

Sims, G. (1987b). Famous Scientists. Cypress, CA: Creative Teaching Press.

Sinoway, L. I., Musch, J. R., Minotti, R., \&, \& Zelis, R. (1986). Enhanced maximal metabolic vasodilation in the dominant arm of tennis players. Journal of Applied Physiology, 61(673-678).

Slavit, D.H., \& McCaffrey, T.V. (1991). Regulation of phonatory efficiency by vocal fold tension and glottic width in the excised canine larynx. Annals of Otology, Rhinology, and Laryngology, 100, 668-677.

Smith, E., Gray, S. D., Dove, H., Kirchner, L., \& Heras, H. (1997). Frequency and effects of teachers' voice problems. $J$ Voice, 11(1), 81-87.

Smith, E., Kirchner, H. L., Taylor, M., Hoffman, H., \& Lemke, J. H. (1998). Voice problems among teachers: differences by gender and teaching characteristics. $J$ Voice, 12(3), 328334.

Solomon, N. P. (2007). Vocal fatigue and its relation to vocal hyperfunction. International Journal of Speech-Language Pathology, 10(4), 1-13.

Solomon, N. P., Glaze, L. E., Arnold, R. R., \& van Mersbergen, M. (2003). Effects of a vocally fatiguing task and systemic hydration on men's voices. $J$ Voice, 17(1), 31-46.

St Clair Gibson, A., Lambert, E. V., Rauch, L. H., Tucker, R., Baden, D. A., Foster, C., et al. (2006). The role of information processing between the brain and peripheral physiological systems in pacing and perception of effort. Sports Med, 36(8), 705-722.

St Clair Gibson, A., \& Noakes, T. D. (2004). Evidence for complex system integration and dynamic neural regulation of skeletal muscle recruitment during exercise in humans. $\mathrm{Br} J$ Sports Med, 38(6), 797-806.

Stemple, J. C., Stanley, J., \& Lee, L. (1995). Objective measures of voice production in normal subjects following prolonged voice use. J Voice, 9(2), 127-133.

Titze, I. R. (1988). The physics of small amplitude oscillation of the vocal folds. Journal of the Acoustical Society of America, 83(4), 1536-1552.

Titze, I.R. (1992). Vocal efficiency. Journal of Voice, 6, 135-138.

Titze, I. R. (1999). Toward occupational safety criteria for vocalization. Logoped Phoniatr Vocol, 24, 49-54.

Titze, I.R. (2006). Voice training and therapy with a semi-occluded vocal tract: rationale and scienific underpinnings. Journal of Speech, Language, and Hearing Research, 49, 448459.

Tomlin, D. L., \& Wenger, H. A. (2001). The relationship between aerobic fitness and recovery from high intensity intermittent exercise. Sports Med, 31(1), 1-11. 
Van den Berg, J. (1958). Myoelastic-aerodynamic theory of voice production. Journal of Speech and Hearing Research, 1(3), 227-244.

Verdolini, K., Druker, D.G., Palmer, P.M., \& Samawi, H. (1998). Laryngeal adduction in resonant voice. Journal of Voice, 12 (3), 315-327.

Verdolini, K., \& Ramig. (2001). Review: Occupational risks for voice problems. Logopedics Phoniatrics Vocology, 26 (1), 37-46.

Verdolini, K., Rosen, C. A., \& , \& Branski, R. C. (2006). Classification Manual of Voice Disorders- I. . Mahwah, NJ: Erlbaum.

Vollestad, N. K. (1997). Measurement of human muscle fatigue. Journal of Neuroscience Methods, 74(2), 219-227.

Waters, R.L., Hislop, H.J., Perry, J., Thomas, L., \& Campbell, J. (1983). Comparative cost of walking in young and old adults. Journal of Orthopaedic Research, 1 (1), 73-76.

Weir, J. P., Beck, T. W., Cramer, J. T., \& Housh, T. J. (2006). Is fatigue all in your head? A critical review of the central governor model. Br J Sports Med, 40(7), 573-586; discussion 586.

Welham, N. V., \& Maclagan, M. A. (2003). Vocal fatigue: current knowledge and future directions. $J$ Voice, 17(1), 21-30.

Welham, N. V., \& Maclagan, M. A. (2004). Vocal fatigue in young trained singers across a solo performance: a preliminary study. Logoped Phoniatr Vocol, 29(1), 3-12.

Westerblad, H., \& Allen, D. G. (2002). Recent advances in the understanding of skeletal muscle fatigue. Curr Opin Rheumatol, 14(6), 648-652.

Westerblad, H., \& Allen, D. G. (2003). Cellular mechanisms of skeletal muscle fatigue. Adv Exp Med Biol, 538, 563-570; discussion 571.

Westerblad, H., Allen, D. G., \& Lannergren, J. (2002). Muscle fatigue: lactic acid or inorganic phosphate the major cause? News Physiol Sci, 17, 17-21.

Westerblad, H., Lee, J. A., Lannergren, J., \& Allen, D. G. (1991). Cellular mechanisms of fatigue in skeletal muscle. Am J Physiol, 261(2 Pt 1), C195-209.

Wooton, S. A., \&, \& Williams, C. (1983). The influence of recovery duration on repeated maximal sprints. In H. G. Knuttgen, Vogel, J.A., \& Poortmans. J. (Ed.), Biochemistry of Exercise (pp. 269-273). Champaign, IL: Human Kinetics. 